WALDEN

UNIVERSITY

A higher degree. $A$ higher purpose.

Walden University ScholarWorks

2017

\title{
Evaluating the Impact of Integrated Care on Service Utilization in Serious Mental Illness
}

Heidi C. Waters

Walden University

Follow this and additional works at: https://scholarworks.waldenu.edu/dissertations

Part of the Health and Medical Administration Commons, Psychiatric and Mental Health Commons, and the Public Policy Commons

This Dissertation is brought to you for free and open access by the Walden Dissertations and Doctoral Studies Collection at ScholarWorks. It has been accepted for inclusion in Walden Dissertations and Doctoral Studies by an authorized administrator of ScholarWorks. For more information, please contact ScholarWorks@waldenu.edu. 


\title{
Walden University
}

\author{
College of Health Sciences
}

This is to certify that the doctoral dissertation by

\author{
Heidi Waters
}

has been found to be complete and satisfactory in all respects, and that any and all revisions required by the review committee have been made.

\author{
Review Committee \\ Dr. Michael Furukawa, Committee Chairperson, Health Services Faculty \\ Dr. Shari Jorissen, Committee Member, Health Services Faculty \\ Dr. Ronald Hudak, University Reviewer, Health Services Faculty
}

\section{Chief Academic Officer \\ Eric Riedel, Ph.D.}

\section{Walden University}

2017 


\begin{abstract}
Evaluating the Impact of Integrated Care on Service Utilization in Serious Mental Illness by
\end{abstract} Heidi C. Waters

MBA, LaSalle University, 2003

MSEd, University of Pennsylvania, 1993

BS, Wheaton College, 1991

\author{
Dissertation Submitted in Partial Fulfillment \\ of the Requirements for the Degree of \\ Doctor of Philosophy \\ Health Services
}

Walden University

January 2017 


\begin{abstract}
Serious mental illness (SMI) affects 5\% of the United States population and is associated with increased morbidity and mortality. Use of high-cost healthcare services is common, including hospitalizations and emergency department (ED) visits. Integrating behavioral and physical healthcare may improve care for consumers with SMI, but prior research findings have been mixed. This quantitative retrospective cohort study addressed the impact of integrated care on physical health and ambulatory care sensitive (ACS) utilization via a program evaluation of an integrated health clinic (IHC) at a community mental health center $(\mathrm{CMHC})$. The research questions assessed whether there was a predictive relationship between IHC enrollment and physical health and ACS-specific service utilization for consumers with SMI when controlling for demographic characteristics and disease severity. Secondary administrative healthcare data, including authorization and electronic medical record data, were provided by the CMHC. Logistic regressions assessed the odds of experiencing an inpatient admission or ED visit before or after IHC enrollment; the predictive relationship between IHC enrollment and service utilization was assessed using multiple linear and Poisson regression analyses. There was no statistically significant impact of integrated care clinic enrollment on physical health or ACS-specific utilization. The sample had lower levels of physical health utilization than would have been expected. In terms of positive social change, results may help the CMHC assess the IHC program, overall clinic success, and use of data. Since policy and payment structures continue to support integrated care models, further research on different programs are encouraged, as each setting and practice pattern is unique.
\end{abstract}


Evaluating the Impact of Integrated Care

on Service Utilization in Serious Mental Illness

by

Heidi C. Waters

MBA, LaSalle University, 2003

MSEd, University of Pennsylvania, 1993

BS, Wheaton College, 1991

Dissertation Submitted in Partial Fulfillment

of the Requirements for the Degree of

Doctor of Philosophy

Health Services

Walden University

January 2017 


\section{Dedication}

This dissertation is dedicated to my father, John L. Thompson, for believing in me and letting me know how brave he thought I was. About 20 years ago as we were sitting in the stands at a rodeo in Durango Colorado, I asked him what he thought my future would hold. At the time, I was struggling to complete a doctoral program at another University. He told me that he thought I would complete my study not because I wanted to but because I thought I had to... Well, I never did. This time I completed my Dissertation because I wanted to, not because I thought I should. It has been a much more rewarding experience. My father gave me the strength to accomplish anything, and I know he would be proud if he were here today. Love you, Dad! 


\section{Acknowledgments}

First and foremost, I owe a debt of gratitude to my data partner CMHC for providing the data for this study and being such a wonderful data partner during the process. Specifically, I would like to thank their Chief Executive Officer for her support, insights, and willingness to allow her clinic to participate as a data partner for this study. I would also like to thank the Chief Operations Officer and Data Analyst for their unending patience with my many questions about the data.

I would also like to thank Dr. Michael Furukawa, my Dissertation Chair and Dr. Shari Jorissen, my Dissertation Faculty Member for their support, insights, and encouragement. I feel lucky to have had these two wonderful mentors during the process, and have learned so much from both of them.

Finally, I would like to thank my family for their encouragement during this process. I could not have made it through this process without the love and support of my fabulous husband, Robert Waters, who lifted me up when wanted to quit, cheered for me as I pushed forward, and always let me know that he was proud of me. Thanks also to my brother, Christian Thompson, who provided helpful insights from his own PhD journey, and my mother, Helen Thompson, for her encouragement. 


\section{Table of Contents}

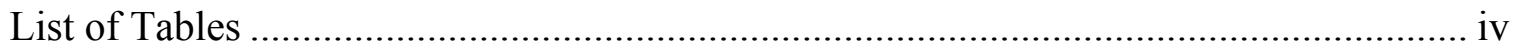

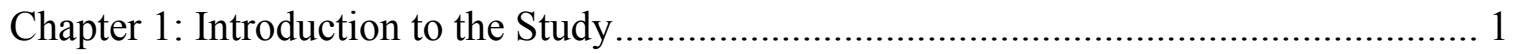

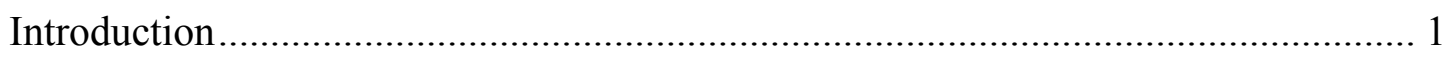

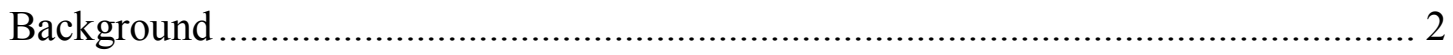

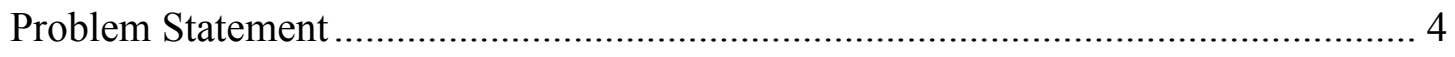

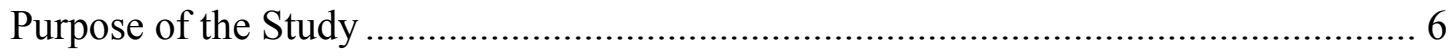

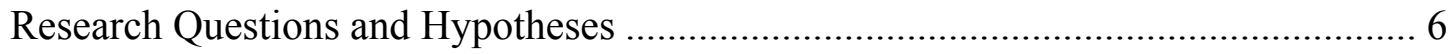

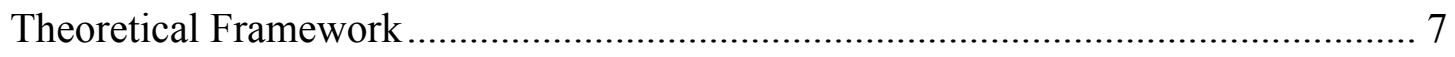

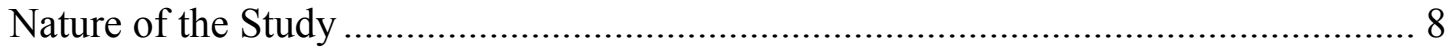

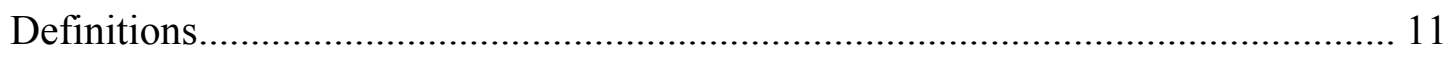

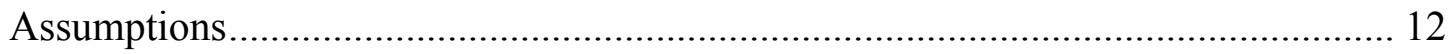

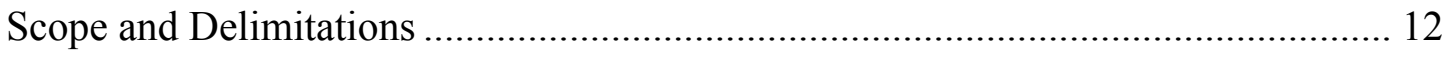

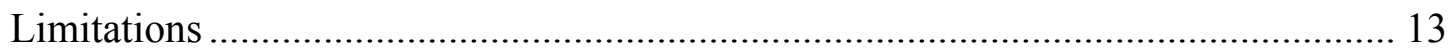

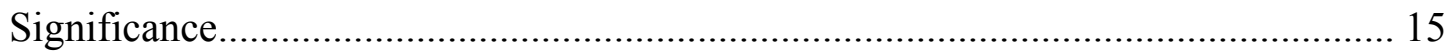

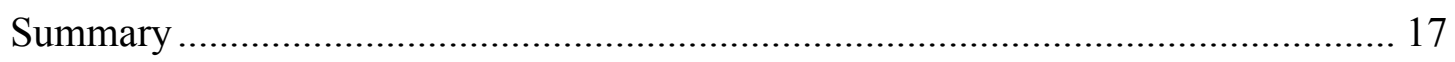

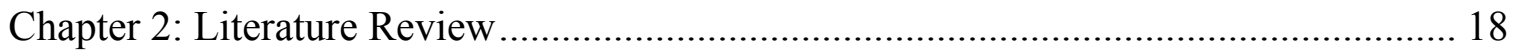

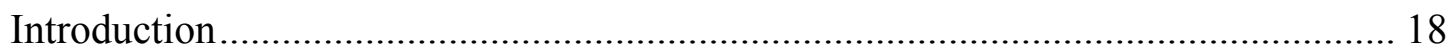

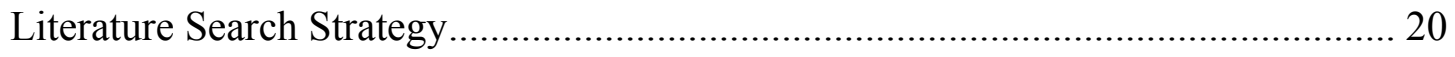

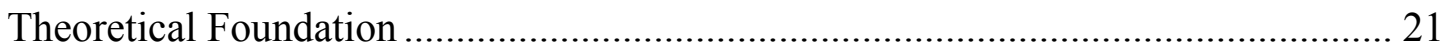

Literature Review Related to Key Variables and Study Concepts .......................... 25

Integrating Physical and Behavioral Healthcare ............................................. 28 


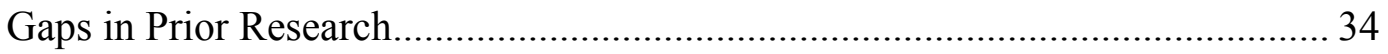

Literature Related to Variables ....................................................................... 36

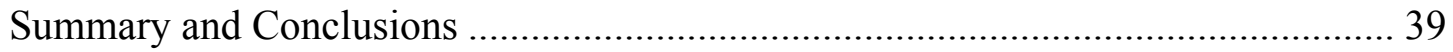

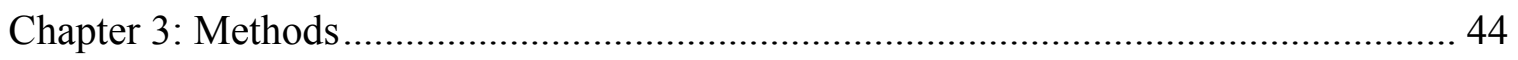

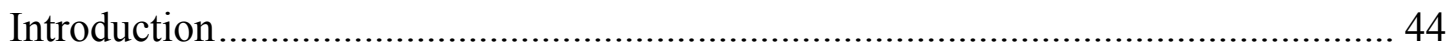

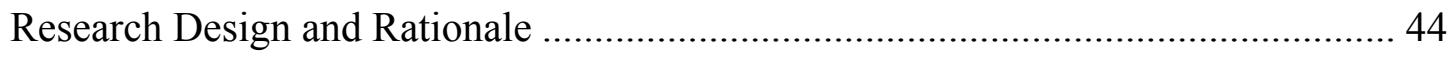

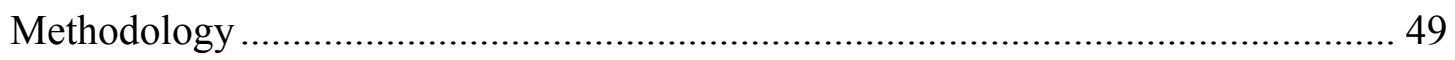

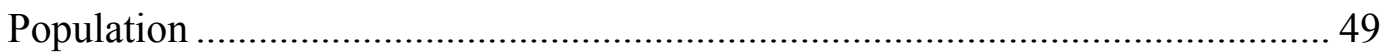

Sampling and Sampling Procedures ........................................................ 50

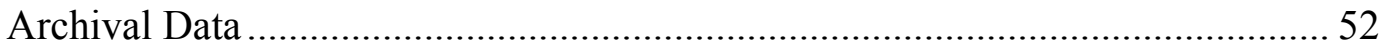

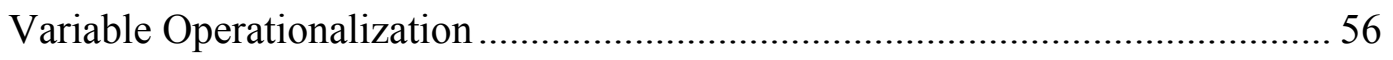

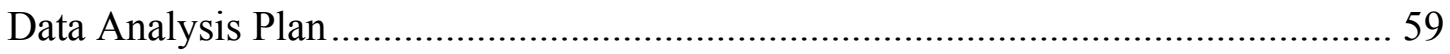

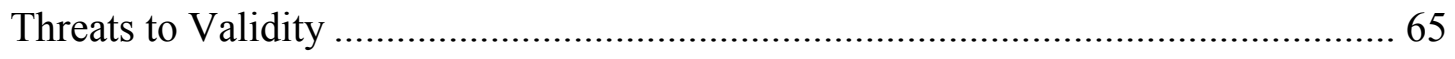

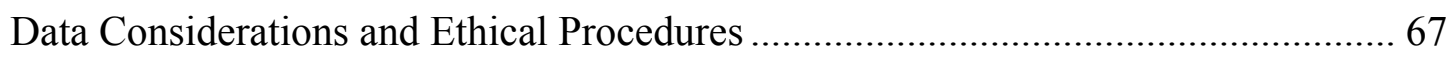

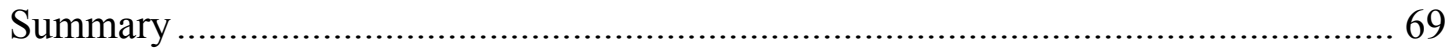

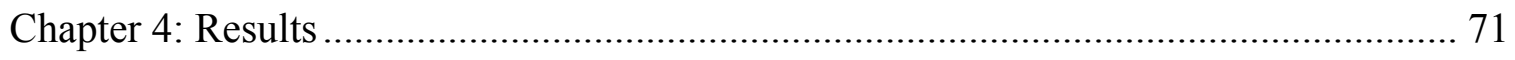

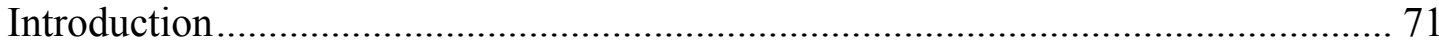

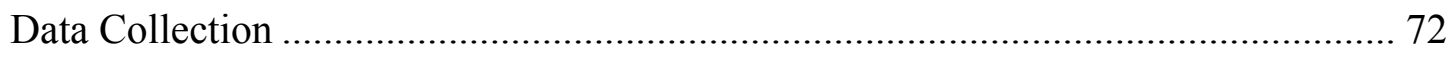

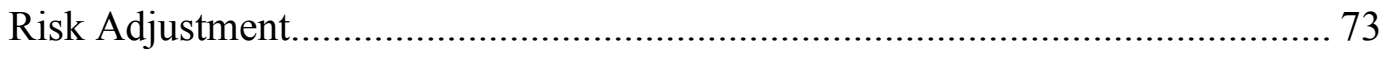

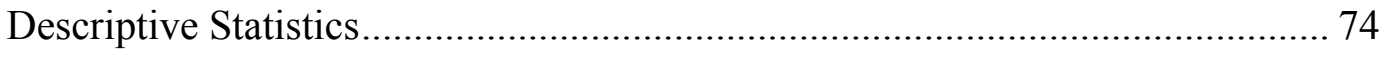

Results 75

Research Question 1: Overall Physical Health Service Utilization ..................... 75 
Research Question 2: ACS-Specific Service Utilization 89

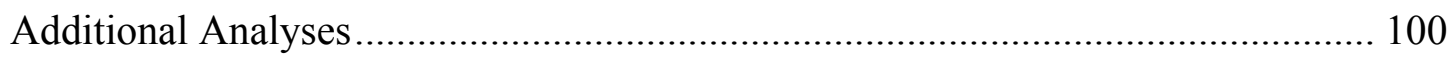

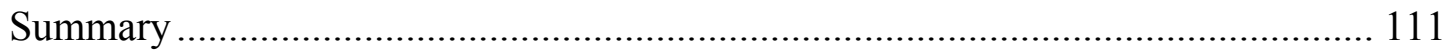

Summary: Research Question 1 - Physical Health Service Utilization ................ 113

Summary: Research Question 2 - ACS-Specific Service Utilization .................. 114

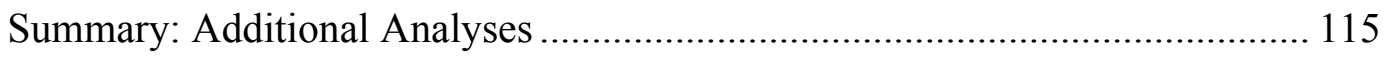

Chapter 5: Discussion, Conclusions, and Recommendations .......................................... 118

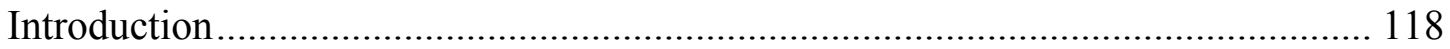

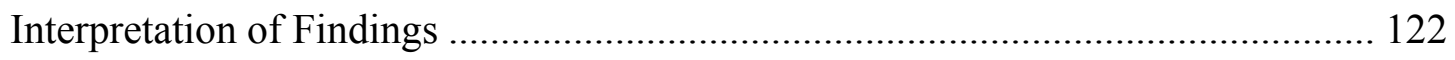

IHC Impact on Utilization ........................................................................... 125

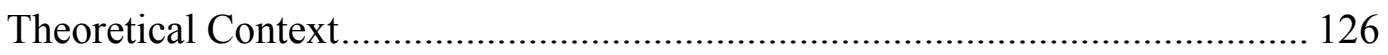

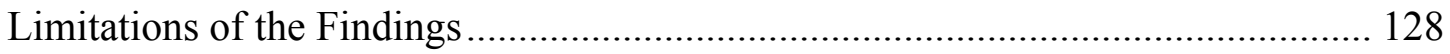

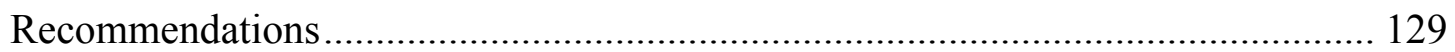

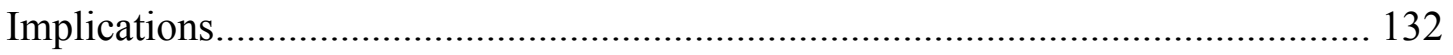

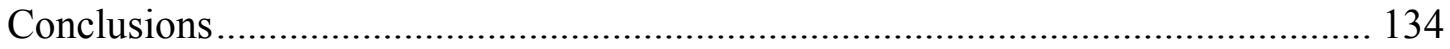

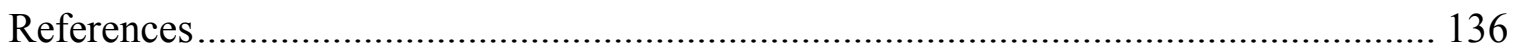

Appendix A: Business Associate Agreement (Redacted).............................................. 156

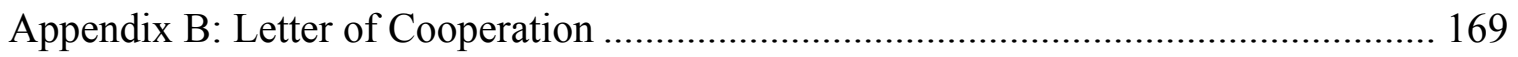

Appendix C: Confidentiality Agreement ................................................................. 170

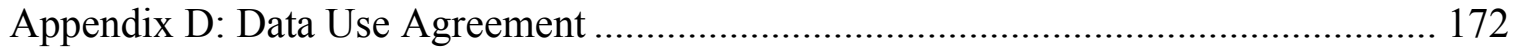




\section{List of Tables}

Table 1. ACS Conditions...........................................................57

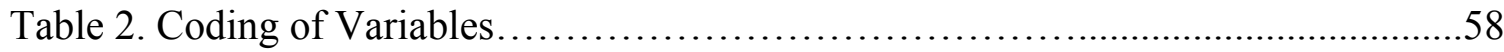

Table 3. Charlson Comorbidity Index Coding and Weighting.......................62

Table 4. Baseline Demographic Characteristics................................73

Table 5. Risk Stratification Variables........................................... 74

Table 6. Pre- and Post-IHC Enrollment Utilization............................... 75

Table 7. Odds Ratio for Experiencing Any Physical Health Inpatient Admission.........78

Table 8. Odds Ratios for Experiencing Any Physical Health ED Visit..................79

Table 9. Individual Predictors of Physical Health Inpatient Admissions................81

Table 10. Individual Predictors of Physical Health Inpatient Days....................83

Table 11. Individual Predictors of Physical Health ED Visits........................84

Table 12. Summary of Regression Analyses for RQ1 .................................87

Table 13. Odds Ratios for Experiencing an ACS-specific Inpatient Admission...........99

Table 14. Individual Predictors of ACS-specific Inpatient Admissions..................94

Table 15. Individual Predictors of ACS-specific Inpatient Days.....................96

Table 16. Individual Predictors of ACS-specific ED Visits........................97

Table 17. Summary of Regression Analyses for RQ2 .............................97

Table 18. Pre- and Post-Enrollment Utilization Descriptive Statistics: Additional Variables............................................................ 100

Table 19: Summary of Regression Analyses for All-Cause Utilization..........................104

Table 20. Summary of Regression Analyses for Mental Health Utilization..................109 
Chapter 1: Introduction to the Study

Introduction

The problem I addressed in this quantitative retrospective cohort study was the lack of evidence on the impact of collocated integrated physical healthcare within the community behavioral health setting on healthcare utilization for physical health conditions among consumers with serious mental illness (SMI). This study was a program evaluation of an integrated health clinic (IHC) collocated within a large community mental health center (CMHC). The main outcome variables of interest were inpatient and emergency department (ED) utilization pre- and post-IHC enrollment. Recent changes in the payer landscape indicated a move toward quality-based reimbursement rather than quantity-based reimbursement. Therefore, an understanding of the effectiveness of integrated care programs and their ability to reduce cost of care and improvement of quality will be important (Burwell, 2015). From a practical perspective, results from this study may help leaders at the CMHC assess the success of the IHC program and identify potential changes to optimize outcomes. As integrated care models become more common, assessments of various care models will improve the level of evidence available to inform optimal care for this population.

In this chapter, I provide a background and overview of the study, including the scope of the problem to be addressed, gaps in the current literature, and need for this study. The study design, research questions, hypotheses, and variables are then introduced. The theoretical underpinning of the study, the chronic care model (CCM), will be discussed briefly, highlighting its relevance to the current study. Important terms 
will be defined and the study assumptions, delimitations, and limitations will be outlined. Lastly, the significance of the study will be explained.

\section{Background}

SMI affects almost 5\% of the United States population (Substance Abuse and Mental Health Services Administration [SAMHSA], 2013). Consumers with SMI die 1520 years earlier than people without SMI (Thornicroft, 2011). This population also has higher rates of comorbid physical illness, including cardiovascular disease and diabetes, due in part to lifestyle factors, medication side effects, and suboptimal physical healthcare (De Hert et al., 2011; Mitchell \& Lawrence, 2011; Thornicroft, 2011). Use of high-cost healthcare services is also common among consumers with SMI, including inpatient hospitalizations with long stays and ED visits (Heslin \& Weiss, 2015). Multiple chronic comorbidities have been associated with high spending among Medicaid and Medicare dual-eligible consumers with mental health disorders (Frank \& Epstein, 2014).

Integrating behavioral and physical healthcare may help improve care for consumers with SMI (Druss, Rohrbaugh, Levinson, \& Rosenheck, 2001; Frank \& Epstein, 2014), but the degree of coordination or integration is important. Heath and colleagues (2013) described six levels of care, ranging from cross-referrals to co-location without joint treatment planning, through fully integrated care involving joint treatment planning and integrated medical records (Heath, Wise Romero, \& Reynolds, 2013). Cross-referrals often prove ineffective because patients rarely reach the referral site (Manderscheid \& Kathol, 2014). Researchers assessing collocated models have primarily focused on behavioral health clinicians collocated in primary care facilities treating 
depression; these studies have shown positive outcomes (Druss et al., 2001; Solberg et al., 2013; Thiejke, Vannoy, \& Unutzer, 2007).

Optimal care integration involves development and implementation of combined physical and mental health treatment plans (Gerrity, 2015). Researchers assessing integrated care programs have rarely focused on SMI, co-location in behavioral health clinic, or collaborative care that includes joint treatment planning (Gerrity, 2015). Since the majority of persons with SMI seek care at behavioral health clinics, this setting may be the more appropriate setting for integrated care programs serving consumers with SMI (Manderscheid \& Kathol, 2014). A few effective models of collocated care for consumers with SMI exist, but the studies have been conducted within the Veteran's Health Administration (VHA) and do not involve joint treatment planning (Pirraglia et al., 2012; Randall, Mohr, \& Maynard, 2014; Schaps \& Post, 2015). There is a gap in knowledge regarding such programs in community mental health settings.

The RAND Corporation recently reviewed 56 programs funded by the Substance Abuse and Mental Health Services Administration (SAMHSA) under the Primary and Behavioral Health Care Integration grant program. The authors of the review described challenges faced by models of physical health collocated in behavioral health settings including culture, space, consent to treatment and information sharing, maintenance of medical records, and referral processes. The review yielded only one study that demonstrated a reduction in ED visits and an increase in screening for hypertension and diabetes (Schaps \& Post, 2015). However, this program involved training psychiatrists to provide additional medical care, rather than integrating a medical specialist into the 
behavioral health setting. One example of a truly integrated care program was provided in the review, but no results were available from this system at the time of publication (Schaps \& Post, 2015).

Recent changes in health policy address the need for improving care for consumers with SMI and suggest financing structures for integrated care pilots (Barry \& Huskamp, 2011; Druss \& Mauer, 2010; SAMHSA, 2014). It will be important to assess how integrated care programs perform in terms of reducing costly inpatient and ED utilization, since payers are moving toward reimbursing providers on the basis of quality rather than quantity of services (Burwell, 2015). In this study, I focused on the impact of integrated care and collaborative treatment planning on high-cost service utilization for consumers with SMI in a community-based mental healthcare setting.

\section{Problem Statement}

In this study I addressed the lack of evidence about the impact of integrated care on overall physical health and ambulatory care sensitive (ACS)-specific utilization. Integrated care involving the development and implementation of combined physical and mental health treatment plans is necessary in providing high-quality care for consumers with SMI (Gerrity, 2015). However, few effective models of integrated care within community behavioral health settings exist (Collins, Hewson, Munger, \& Wade, 2010; Gerrity, 2015). Recently several healthcare policies, including the Patient Protection and Affordable Care Act (PPACA), have been implemented; they emphasize quality of care for consumers with SMI and provide potential financing structures for integrated care pilots (Barry \& Huskamp, 2011; Druss \& Mauer, 2010; SAMHSA 2014). SAMHSA has 
also established the Primary and Behavioral Health Care Integration Program that funds coordinated and integrated services through the colocation of primary and specialty care medical services in community-based behavioral health settings (SAHMSA, 2014). It will be important to understand how these integrated care programs perform in terms of reducing costly inpatient admissions and ED services as payers move toward reimbursement of providers based on quality rather than quantity of services provided (Burwell, 2015).

For a majority of consumers with SMI, the behavioral health clinic serves as the primary, and sometimes only, provider of healthcare. As a result, these clinics may be the best setting in which to integrate physical and behavioral healthcare (Kilbourne, Welsh, Mccarthy, Post, \& Blow, 2008; Manderscheid \& Kathol, 2014). Use of collaborative approaches to patient care is common in behavioral health settings because care managers can help coordinate the sharing of information among the treatment team, engage patients in their treatment, encourage adherence to treatment plans, track outcomes, and monitor progress (Norquist, 2014). The majority of studies that have assessed collocated primary care within a behavioral health setting have taken place in the Veterans Health Administration (VHA) system; however, results of these studies were mixed (Kilbourne, Welsh, McCarthy, Post, \& Blow, 2008; Kilbourne et al., 2011). Therefore, in this study I addressed the lack of evidence about the impact of integrated care on overall physical health and ACS-specific utilization. 


\section{Purpose of the Study}

The purpose of this quantitative study was to assess the impact of integrated care on overall physical health and ACS inpatient and ED utilization when controlling for demographic characteristics and disease severity for consumers with SMI through evaluation of an IHC program at one large CMHC.

\section{Research Questions and Hypotheses}

RQ1: What is the predictive relationship, if any, between IHC enrollment and overall physical health utilization for consumers with SMI when controlling for demographic characteristics and disease severity?

H01: There is no statistically significant predictive relationship between IHC enrollment and overall physical health service utilization (number of inpatient admissions and ED visits, number of inpatient days) when controlling for demographic characteristics (age and gender) and disease severity (primary psychiatric diagnosis, disease burden).

$\boldsymbol{H A 1 :}$ There is a statistically significant predictive relationship between IHC enrollment and overall physical health service utilization (number of inpatient admissions and ED visits, number of inpatient days) when controlling for demographic characteristics (age and gender) and disease severity (primary psychiatric diagnosis, disease burden).

RQ2: What is the predictive relationship, if any, between IHC enrollment and ACS-specific service utilization for consumers with SMI when controlling for demographic characteristics and disease burden? 
H02: There is no statistically significant predictive relationship between IHC enrollment and ACS-specific service utilization (number of inpatient admissions and ED visits, number of inpatient days) when controlling for demographic characteristics (age and gender) and disease severity (primary psychiatric diagnosis, disease burden).

HA2: There is a statistically significant predictive relationship between IHC enrollment and ACS-specific service utilization (number of inpatient admissions and ED visits, number of inpatient days) when controlling for demographic characteristics (age and gender) and disease severity (primary psychiatric diagnosis, disease burden).

\section{Theoretical Framework}

The theory most applicable to this study was the chronic care model (CCM). The CCM focuses on the need for collaboration when providing healthcare services and includes five aspects directly related to high quality care; (a) use of explicit care plans, (b) reorganization of healthcare practices to better meet patient needs, (c) the ability to identify patient needs, particularly related to information and behavior change, (d) access to the appropriate clinical expertise, and (e) information systems that support optimal care (Wagner, Austin, \& Von Korff, 1996). Aspects of the CCM related to redesigning healthcare delivery systems and improving clinical information systems align with the concept of integrated physical and behavioral healthcare (McLellan et al., 2014). The CCM also emphasizes the need for collaboration among healthcare professionals to provide adequate care for patients, treating all conditions rather than treating each individual condition in isolation, and involves proactive patient management to prevent serious and expensive relapses (Rush, 2014). The CCM has been used as a basis for prior 
studies in populations with mental health conditions, with mixed results (Bauer et al., 2015; Bradford et al., 2013; Kilbourne et al., 2009; Thota et al., 2012). The CCM and its relevance to the current study will be described in detail in Chapter 2

\section{Nature of the Study}

In this quantitative retrospective cohort design study, I used secondary administrative healthcare data from the $\mathrm{CMHC}$ as the primary data source. The independent variable was enrollment in the IHC. The dependent variables included the number of physical health and ACS-specific inpatient admissions, inpatient days, and ED visits. Covariates included consumer age, gender, initial psychiatric diagnosis, and comorbidity burden. A quantitative research design was chosen because the dependent variables of interest (healthcare resource utilization) were best measured numerically, and the relationship between IHC enrollment and healthcare resource utilization was the aim of the study. Quantitative research methods using administrative healthcare data have successfully measured similar outcomes related to healthcare utilization and cost (Birnbaum et al., 1999). Furthermore, administrative healthcare data have also been used in outcomes research for decades with success in analyzing certain types of outcomes that can be measured with these data sources, including healthcare utilization and cost (Birnbaum et al., 1999).

The independent variable was enrollment in the IHC, which was coded as " 0 " for preenrollment and "1" for postenrollment for each consumer. The dependent variables included the number of inpatient admissions, number of inpatient days, and number of ED visits. Only physical health inpatient admissions and ED visits were included in the 
original analysis, and a further determination was made as to whether the visits were ACS-specific. Covariates included consumer age, gender, initial psychiatric diagnosis, and comorbidity burden. All-cause and mental health inpatient and ED utilization were assessed in a post-hoc analysis.

The sample included all consumers who enrolled in the IHC between October 1, 2013 and June 30, 2015. Several data files from the CMHC were used in this analysis including: (a) CMHC contact files containing consumer demographic information from October 1, 2013 to December 31, 2015 for all consumers of the CMHC; (b) inpatient admission and ED visit authorization data from October 1, 2013 to December 31, 2015 for consumers of the CMHC enrolled in either AmeriGroup or UnitedHealth health plans; and (3) IHC electronic medical record data from October 1, 2013 to December 31, 2015. All data were stored in an SPSS data file for analysis and analyzed using SPSS Statistics for Mac, Student Edition, version 23 (IBM).

I assessed inpatient admissions and ED use for physical health diagnoses, as well as diagnoses classified as ACS-specific, comparing the six months before to the six months following the initial IHC enrollment date. I planned to use paired-sample $t$-tests to calculate whether differences in the means of service utilization outcomes between the pre- and postenrollment timeframe for consumers in the IHC differed significantly (Field, 2013). However, due to the skewed nature of the data, I instead used non-parametric Wilcoxon signed-rank tests to assess statistically significant differences in median utilization. 
I chose to examine the odds of consumers experiencing any inpatient admission or ED visit before and after IHC enrollment through use of logistic regressions. I examined enrollment first as the sole fixed effect. Age, gender, initial psychiatric diagnosis, and Charlson Comorbidity Index (CCI) score were then added to the fixed-effects portion of the models. Separate logistic regression models were run for overall physical health service utilization and ACS-specific service utilization and for both inpatient admissions and ED visits, resulting in a total of four logistic regression models. I used multiple linear regression analyses to examine the predictive relationship between physical health service utilization and IHC enrollment, demographic characteristics, and disease severity. In the forced entry method, predictors were entered into the model at the same time, making no decisions regarding the order that the predictors were added (Field, 2013). Separate multiple regression models were run for overall physical health service utilization and ACS-specific service utilization for each type of service, including inpatient admissions, inpatient days, and ED visits, resulting in a total of six multiple regression models. I examined the predictive relationship between IHC enrollment and physical health service utilization using Poisson regression analyses. Enrollment was coded first as the sole fixed effect, adding age, gender, initial psychiatric diagnosis, and CCI score to the fixed-effects portion of the model. Separate Poisson regression models were run for overall physical health service utilization and ACS-specific service utilization, and for each type of service, including inpatient admissions, inpatient days, and ED visits, resulting in a total of six Poisson regression models. 
For the statistical tests, a $p$-value $>0.05$ was used as the cutoff for statistical significance, with a confidence interval of $95 \%$. Wilcoxon signed-rank tests were used to assess whether there were statistically significant median differences in inpatient admissions, days hospitalized, and ED visits pre- and post-enrollment in the IHC. Logistic regression results were interpreted using odds ratios assessing the odds of inpatient or ED visits occurring in the pre- and post-enrollment timeframe. Multiple linear regression results were interpreted using the $F$-test to assess whether the overall model was statistically significant and was predictive of service utilization while the $t$ statistics for each predictor variable indicated the level of statistical significance of each independent variable.

\section{Definitions}

The following terms were important to the study and may have multiple meanings. The definitions below describe constructs included in the study and the independent and dependent variables as specifically related to this study. A more detailed analysis of coding, etc. is provided in Chapter 3.

Ambulatory care sensitive conditions: These are conditions that could have been treated in an outpatient setting if appropriate access to primary care were available (Anderson \& Knickman, 2001).

Comorbidity burden: Comorbidity burden was assessed according to the Charlson Comorbidity Index (CCI), which identified 17 comorbid conditions predictive of mortality (Charlson, Wells, Ullman, King, \& Shmukler, 2014; D’Hoore, Bouckaert, \& Tilquin, 1996). 
Integrated care: Integrated care involves the development and implementation of combined physical and mental health treatment plans by collocated providers (SMI; Gerrity, 2015).

Initial psychiatric diagnosis: Initial psychiatric diagnosis was categorized according to prior research as psychotic disorder, mood disorder, or substance abuse disorder in combination with another psychiatric diagnosis (Ettner, Frank, McGuire, Newhouse, \& Notman, 1998; Ettner, Frank, Mark, \& Smith, 2000).

Serious mental illness: SMI was defined as current or past-year presence of any disorder in the Diagnostic and Statistical Manual of Mental Disorders, Fourth Edition (DSM-IV) that causes functional impairment or considerably interferes with one or more life activities among adults 18 years of age or older (SAMHSA, 2013).

\section{Assumptions}

The major assumption made at the outset of this study was that the secondary data received from the CMHC were authentic, not falsified, and reflected care patterns of consumers at the CMHC. Since I did not collect the data myself, I had to assume that the data for the study were reliable. This was a reasonable assumption to make, given that the original purpose of these data was to guide care for consumers of the CMHC.

\section{Scope and Delimitations}

The study sample was limited to consumers of one large CMHC who self-selected for enrollment in an IHC program. Analyses were limited to consumers age 18 years and older for whom six months of data before and after initial enrollment in the IHC were available. I used secondary data to assess whether enrollment in the IHC was associated 
with changes in physical health inpatient and ED service utilization. There may have been other important indicators of IHC success or confounding variables affecting service utilization patterns that were not accounted for and could not be assessed using administrative secondary data. However, using administrative data for this study may allow others to more closely replicate the methodology with other integrated care programs using similar sources of data (Frankfort-Nachmias \& Nachmias, 2008).

While the CCM was chosen as the main theoretical underpinning of this study, systems theory may also have been applicable in explaining the lack of integration between physical and behavioral healthcare providers. Complex-adaptive systems are those in which diverse parts of a system must operate together (Tsasis, Evans, \& Owen, 2012). However, continuous change in the organization creates a lack of predictability (Tsasis, Evans, Rush, \& Diamond, 2013). When applied to integrated care, systems theory may explain why, due to historical practice patterns and the patients they treat, mental health and primary care delivery systems have evolved differently over time and have not been able to coordinate effectively to meet patient needs (Thiejke et al., 2007).

\section{Limitations}

This study was subject to five limitations, (a) the data sources used and the scarcity of utilization data among the sample. While use of administrative data for this study was appropriate, there were fewer inpatient admissions and ED visits than expected and this affected interpretation of the results. (b) A second limitation of this study was that data were specialized. Inpatient and ED utilization were assessed via authorization data sent to the CMHC by two of the health plans to help the CMHC better manage its 
consumers. A third payer did not send such authorization data and there may have been differences in service utilization between health plans that could not be ascertained. Furthermore, the sharing of real-time authorization data between a health plan and a CMHC is probably atypical and therefore may limit the ability of other researchers to reproduce the exact methodology used in the current study. (c) Another limitation related to data was that different people involved in the consumer's care entered the data used in the analysis into the administrative systems. Data entry errors may have occurred leading to inaccuracies in the data or missing data. (d) A further limitation had to do with the study methodology. Since I chose a retrospective cohort design with a convenience sample, bias was introduced. (e) A related limitation to predictive validity was that of selection effects. Since clients at the CMHC self-selected into the IHC, there were likely differences between the study group and other consumers of the CMHC and consumers with SMI in general (Frankfort-Nachmias \& Nachmias, 2008). Therefore, results were not generalizable to a population beyond the consumers of the particular CMHC who enrolled in the IHC program (Mann, 2003; Phua, 2007). However, this study added to the body of literature about collocated integrated care programs in general. Study sample retention was initially thought of as a threat to internal validity as well. Since the SMI population is transient, it was important to assess and account for attrition (FrankfortNachmias \& Nachmias, 2008). However, the consumers at the CMHC were covered by Medicaid and not as likely as consumers covered by commercial insurance to switch health plans or move to other locations. This threat was reduced by limiting the sample to those with a full six months of data pre- and post-enrollment in the IHC. 


\section{Significance}

First and foremost, the results from this study may help leaders at the data partner CMHC assess the success of the IHC program and clinic services in general, and identify opportunities for improvement. Since this study was a program assessment of a particular IHC program, the most immediate results will be realized there. However, the findings may also help inform other providers interested in implementing integrated physical and behavioral health programs by providing evidence to better inform decisions prior to spending resources during implementation. Authors of a recent meta-analysis of interventions for medical conditions among consumers with SMI found low to medium levels of evidence supporting these interventions, and called for further research on implementation strategies in real-world settings (McGinty, Baller, Azrin, Juliano-Bult, \& Daumit, 2016). The current study may help fill this need.

From a policy perspective, this study may also inform future health policy and assist payers in developing value-based payments. Recent implementation of new health policies and grant programs have placed emphasis on quality of care for consumers with SMI and provision of financing structures for integrated care pilots (Barry \& Huskamp, 2011; Druss \& Mauer, 2010; SAMHSA, 2014). As payers in the United States move toward quality-based reimbursement rather than quantity-based reimbursement, an understanding of the effectiveness of these programs in the reduction of cost and improvement of quality will be important (Burwell, 2015).

Apart from policy and practice implications, this study may make an important contribution to the literature. Researchers leading two studies within the VHA evaluated 
the impact of coordinated care programs on service utilization. Neither of these studies showed significant differences in ED service utilization. There are also important differences between the two VHA studies and the current study. While authors of one study assessed veterans with SMI, the sample size was small, which may have affected the results (Pirraglia et al., 2012). Researchers involved in the second study assessed veterans with PTSD, rather than SMI (Randall et al., 2014), and the etiologies and treatment paradigms for these conditions differed markedly. Additionally, the intervention in that study involved health practitioners as part of a care team, not an integrated care model in a behavioral health clinic setting (Randall et al., 2014; Veterans Health Administration, 2015). Furthermore, generalizability to other populations may be limited due to use of EMR data in the VHA and the fact that the VHA serves a selected group of consumers who are likely not reflective of the general United States population (Goulet et al., 2007; Pirraglia et al., 2012). Therefore, in this study, I addressed integrated care in a community mental health center, which is likely more reflective of the United States population than the VHA studies.

Another important outcome of this study relates to the social change implications for consumers with SMI. As integrated care models become more common, researchers assessing the various care models will improve the level of evidence available to inform optimal care for this population. While consumers with SMI often do not participate in traditional health home models (Lichstein et al., 2014), other models of care that are located within community behavioral healthcare settings may be more appropriate, thus 
increasing access to physical healthcare and improving outcomes among the SMI population (Barry \& Huskamp, 2011).

\section{Summary}

SMI is associated with high rates of mortality and comorbid physical illness and is associated with use of high-cost healthcare services including inpatient hospitalizations and ED visits. Integrating behavioral and physical healthcare may improve care for consumers with serious mental illness, but findings of prior research have been mixed. This quantitative retrospective cohort study addressed the lack of evidence about the impact of integrated care on overall physical health and ACS-specific utilization through a program evaluation of an IHC. The main research question assessed whether there was a predictive relationship between IHC participation and physical health or ACS-specific service utilization for consumers with SMI. The analyses controlled for demographic characteristics and disease severity. The study has implications for the CMHC implementing the IHC program as well as for policy makers assessing new models of care funded under the ACA.

In Chapter 2, I expand upon the concepts presented here, and include an explanation of the CCM and its application to this study, an overview of SMI and its various implications to the United States healthcare system, and different models of integrated care with a focus on the model used by the data partner. I highlight gaps in prior research, point to a need for the current study, and explore the literature on the variables used in this study. 
Chapter 2: Literature Review

\section{Introduction}

While integrated care has been defined in multiple ways, for the purposes of this

study, I defined it as team-based care, including the development and implementation of combined physical and mental health treatment plans, rather than a simple colocation of services. Joint treatment planning may be necessary to provide the best care for consumers with serious mental illness (SMI; Gerrity, 2015). Researchers have assessed integrated care models within the Veteran's Health Administration (VHA) system (Kilbourne, Lai, Bowersox, Pirraglia, \& Bauer, 2011; Pirraglia et al., 2012); however, there are few effective models of integrated care collocating a physical health clinic in the community behavioral health setting (Collins, Hewson, Munger, \& Wade, 2010; Gerrity, 2015).

The problem I addressed in this study was the lack of evidence assessing the impact of collocated integrated physical healthcare within the behavioral health setting on physical health utilization in a community setting. In this study, I assessed the impact of integrated care on physical health inpatient and emergency department (ED) utilization for consumers with SMI. I specifically evaluated the effects of an integrated health clinic (IHC) collocated within a large community mental health center (CMHC). The main research question I addressed in this study was: What is the predictive relationship, if any, between IHC enrollment and physical health service utilization (both overall and ambulatory care sensitive [ACS]-specific) for consumers with SMI when controlling for demographic characteristics and disease severity? 
SMI affects slightly under 5\% of the United States population (SAMHSA, 2013). Consumers with SMI experience higher rates of morbidity and mortality than those without SMI (Thornicroft, 2011). Comorbid physical illnesses commonly experienced by consumers with SMI include cardiovascular disease (CVD) and diabetes, caused in part by lifestyle factors, medication side effects, and suboptimal physical healthcare (De Hert et al., 2011; Mitchell \& Lawrence, 2011; Thornicroft, 2011). The nature of illness of SMI and high rates of comorbidity may contribute to the high cost of healthcare among this population. Consumers with SMI use intensive healthcare resources including inpatient hospitalizations, and as such, the cost of care for SMI is high (Heslin \& Weiss, 2015).

Integrating behavioral and physical healthcare may help improve care for consumers with SMI (Druss, Rohrbaugh, Levinson, \& Rosenheck, 2001; Frank \& Epstein, 2014). While integration of behavioral health within primary care clinics has been studied in the past, these models are not always adequate for consumers with SMI, since the behavioral health clinic serves as their primary care setting (Manderscheid \& Kathol, 2014). Several researchers have assessed the impact of collocated care in both physical and behavioral healthcare settings on access to care, quality of life, and service utilization. However, the majority of these studies have taken place within the VHA system and may not reflect care in community settings (Druss, Rohrbaugh, et al., 2001; Kilbourne et al., 2011; Pirraglia, Kilbourne, Lai, Friedmann, \& O’Toole, 2011). Furthermore, the models of integrated care most commonly studied typically do not include coordinated treatment planning between physical and mental health clinicians (Gerrity, 2015). 
This chapter covers the following topics: (a) a review of the literature search strategies, (b) an overview of the chronic care model (CCM), the theoretical foundation of this study, including a review of past studies applying CCM to mental health populations, (c) the problem of SMI, including incidence, prevalence, morbidity, mortality and healthcare resource use with a focus on high cost services such as inpatient hospitalization and ED visits, (d) different models of integrated care, focusing specifically on the model used by the data partner CMHC, and (e) gaps in prior research, pointing to a need for the current study and presented literature related to the variables used in the current study.

\section{Literature Search Strategy}

Citations for SMI prevalence and specific diagnoses of interest including serious mental illness, SMI, bipolar disorder, schizophrenia, or schizoaffective disorder were based on published review articles. I conducted a search of English-language publications in PubMed Health for dates of publication between 2010 and July 14, 2015 (the day the search was conducted) for integrated care, including integrated care, integrated behavioral care, collaborative behavioral care, and collocated care. When searching for these terms, 705 results were found. When combining a search for integrated care with mental illness, 249 results were found. A similar search using the same dates and English language was also conducted to search for articles regarding the CCM and SMI. The search for chronic care model or CCM yielded 1,321 results, but when combined with mental illness, the results dropped to five, for bipolar disorder the results dropped to 11, and when combined with schizophrenia, results dropped to one. 
Following the initial searches, smaller supplemental searches were also conducted for articles cited in review articles retrieved from the initial search. This search was especially useful in identifying seminal articles about the CCM, which was initially developed in the 1990s (Wagner et al., 1996). Furthermore, weekly searches were conducted to identify recent literature published since the initial search for articles related to integrated behavioral healthcare using Google Scholar and searches of specific journals likely to carry articles of interest, including JAMA Psychiatry, Psychiatric Services, Schizophrenia Research, and Health Affairs.

\section{Theoretical Foundation}

I used the $\mathrm{CCM}$ as the theoretical foundation for this study. Wagner et al. first developed the CCM in the 1990s in response to the focus on acute illness by practitioners within the healthcare system. Therefore, the system did not adequately meet the needs of patients with chronic illness. Practitioners in the healthcare system focused on diagnosing and treating acute illness rather than addressing predictable needs related to chronic conditions. This resulted in suboptimal health outcomes related to an inability to identify complications in a timely manner, failing health due to inadequate assessment and follow-up, inability of the patient to recognize risk factors, suboptimal interventions leading to lower quality of care, and unmanaged patient distress (Wagner et al., 1996). The CCM was developed to provide appropriate evidence-based interventions, educate patients and caregivers about managing illness, and empower patients to better care for themselves (Austin, Wagner, Hindmarsh, \& Davis, 2000). 
The founders identified five aspects directly related to high quality care, including (a) use of explicit care plans, (b) reorganization of healthcare practices to better meet patient needs, (c) the ability to identify patient needs, particularly related to information and behavior change, (d) access to the appropriate clinical expertise, and (e) information systems that support optimal care (Wagner et al., 1996). The CCM as originally theorized contained four components: patient self-management assistance, clinical health information systems, redesign of practices to focus on prevention of disease, and use of evidence-based medicine (Wagner et al., 1996). Two other components were added later, including organizational support and linking the patient to community resources (Bodenheimer T, Lorig K, Holman H, \& Grumbach K, 2002; Bodenheimer T, Wagner EH, \& Grumbach K, 2002).

There are six key components of CCM: (a) focus on chronic conditions and an emphasis on quality improvement and measurement against goals, (b) emphasis on the importance of the role that patients have in managing their care, (c) use of evidence based guidelines in clinical practice, (d) focus on teamwork and an expanded scope of practice for team members to support chronic care, (e) information systems that provide relevant data for clinical decision-making, and (f) use of community supports to help meet patients' needs (Wagner et al.; 2001). While CCM theory in its entirety is important, several key aspects explain the concept behind integrated care and supported the current study assessing integration of physical healthcare into behavioral health settings for consumers with SMI (Woltmann et al., 2012). Specifically, aspects of CCM related to redesigning healthcare delivery systems and improving clinical information systems 
aligned with the concept of integrated physical and behavioral healthcare (McLellan et al., 2014). Care plans have typically involved treatment of a single disease in isolation. However, appropriate care planning involves all conditions experienced by a patient and treating that patient as a whole, which matters greatly in patients who have multiple chronic conditions (Burt et al., 2014). The CCM emphasized the need for collaboration among healthcare professionals to adequately provide care for patients, treating all conditions rather than treating each individual condition in isolation, and involves proactive patient management to prevent serious and expensive relapses (Rush, 2014). This translates into teams of providers caring for patients, where tasks can be delegated among the team based on clinical experience, with coordination and communication between the various team members (Austin et al., 2000). Improved outcomes are related to strong and productive relationships between motivated patients and their teams of healthcare providers acting proactively (Barr et al., 2003).

Successful management of chronic conditions also depends on providers having access to the necessary expertise. In traditional medicine, this is accomplished through referrals from primary care to specialists; however, this system often fragments care because referring physicians and specialists do not communicate effectively. Systems with distributed expertise, in which clinicians consult with one another to provide teambased care, may be more effective in delivering treatment to consumers in need of chronic care (Austin et al., 2000). Use of shared electronic medical record (EMR) systems may facilitate sharing of information between providers and improve prevention of complications (Glasgow, Tracy Orleans, Wagner, Curry, \& Solberg, 2001). The CCM 
is used widely today as a framework for practice improvement, mostly in ambulatory care settings, that helps practice administrators translate ideas for change into specific tactics (Coleman, Austin, Brach, \& Wagner, 2009). The CCM framework could also be used in the development and implementation of collaborative treatment planning between collocated physical and behavioral health providers.

Due to its prior use in care for consumers with SMI, the CCM was chosen as the primary theory for the current study. Through this study, I built on earlier research based on the CCM to improve care for consumers with mental health conditions. Authors of a meta-analysis of 53 studies based on the CCM conducted to improve the quality of life in consumers with mental health disorders found that the majority of CCM-based studies assessed outcomes for consumers with depression in primary care and results indicated a positive impact of the CCM on depression and mental and physical quality of life (Miller et al., 2013). The original four components of the CCM were those most often implemented in the studies assessed, but no single element was statistically associated with success in model implementation (Miller et al., 2013). Authors of a similar literature review also showed improvements in depressive symptoms, response to treatment, reemission at six months, recovery at 12 months, and adherence to medication for consumers with depression (Thota et al., 2012).

The CCM has also been used by researchers as a foundation for programs targeting the SMI population, but with mixed results. Authors of a study in consumers with bipolar disorder showed that a CCM-based program reduced time in bipolar and manic episodes and improved some functional outcomes such as work and familial 
functioning; however, no effects were seen for time in depressive episode, number of symptoms, or marital/social functioning (Bauer et al., 2015). Researchers did show improvement on the Physical Component Score (PCS) of the SF-36 for consumers with bipolar disorder and cardiovascular disease within the VHA system, when implementing a program based on the CCM (Kilbourne et al., 2009). Similarly, authors of a literature review of four studies found mixed results when evaluating the effects of CCM-based care models on the PCS and Mental Component Score (MCS) of the SF-36 (Bradford et al., 2013). Authors of another systematic review of 46 articles demonstrated significant effects in $31.7 \%$ of studies of consumers with bipolar disorder and $47.6 \%$ of studies in multiple disorders. However, there were no favorable results with regard to cost of care reductions (Woltmann et al., 2012b).

\section{Literature Review Related to Key Variables and Study Concepts}

The Substance Abuse and Mental Health Services Administration (SAMHSA) defined SMI as current or past-year presence of any disorder in the Diagnostic and Statistical Manual of Mental Disorders, Fourth Edition (DSM-IV) that causes functional impairment or considerably interferes with one or more life activities among adults 18 years of age or older (SAMHSA, 2013). In 2012, there were approximately 9.6 million adults in the United States living with SMI during the past year, which accounted for $4.9 \%$ of the population (SAMHSA, 2013). Schizophrenia affects about $1 \%$ of the United States population, bipolar disorder affects approximately $2 \%$, and $6 \%$ experience a major depressive episode (Regier et al., 1993; SAMHSA, 2013). 
Authors of a recent meta-analysis assessing mortality rates for persons with mental disorders found the relative risk of death from mental disorders was $2.22 \%$, attributable to both natural and unnatural causes, and a potential of 10 years of life lost (Walker, McGee, \& Druss, 2015). When looking at SMI as a subset of mental disorders, the data are even more concerning. Higher mortality rates among consumers with SMI have been well documented. Men with SMI die on average 20 years younger than their non-SMI counterparts and women die approximately 15 years earlier (Thornicroft, 2011). The risk of premature death was found to be 3 to 3.5 times greater for consumers with schizophrenia and two times higher for consumers with bipolar disorder than the general population (Khan, Faucett, Morrison, \& Brown, 2013; Medici, Videbech, Gustafsson, \& Munk-Jørgensen, 2015; Olfson, Gerhard, Huang, Crystal, \& Stroup, 2015; Perkins, 2015) Higher mortality rates are due to a multitude of reasons. Researchers in one study found that rates of death due to natural causes, accidents, suicide, and comorbid illness were higher in the population of consumers with schizophrenia than the general population (Perkins, 2015), while researchers involved in another study found that cardiovascular disease (CVD), cancer, chronic obstructive pulmonary disease (COPD), and influenza and pneumonia contributed to elevated morbidity rates among consumers with schizophrenia (Olfson et al., 2015).

Comorbid physical illness is common among consumers with SMI, with authors of one study finding that $74 \%$ of consumers with SMI had at least one chronic medical condition, while half were diagnosed with two or more (Jones et al., 2004). The reasons for high levels of comorbidity include side effects of psychiatric medication, unhealthy 
lifestyle, and low rates of screening and suboptimal care for physical health conditions in the SMI population (De Hert et al., 2011; Mitchell \& Lawrence, 2011; Thornicroft, 2011). Only $21 \%$ of consumers with SMI in one study received recommended levels of primary care (Druss et al., 2009) while inadequate levels of screening for physical health conditions including Papanicolaou (Pap) tests and mammograms have been highlighted in other studies (De Hert et al., 2011; Salsberry et al., 2005). Researchers demonstrated that among the VHA population, the presence of a mental health disorder alone, or in combination with a substance abuse disorder, decreased the likelihood of receiving preventive services (Druss, Rosenheck, Desai, \& Perlin, 2002).

When treating metabolic conditions including CVD and diabetes, the evidence is equally concerning. Consumers with SMI were less likely than those without SMI to receive hospital-based care or invasive procedures for CVD or diabetic complications, or to receive prescription medications to treat metabolic conditions according to the authors of one study (Scott, Platania-Phung, \& Happell, 2012). Moreover, consumers with mental illness had fewer invasive coronary interventions after serious cardiac events and an increased likelihood of death from those events than people without mental illness. Once results were adjusted to control for indicators of care quality including medication and smoking cessation counseling, the association was no longer significant (Scott, PlataniaPhung, \& Happell, 2012). This may indicate that lower quality of care received by consumers with SMI may greatly contribute to excess mortality in this population (Druss, Bradford, Rosenheck, Redford, \& Krumhold, 2001; Mitchell \& Lawrence, 2011). 
Antipsychotic medication has been associated with cardiometabolic side effects including diabetes, obesity, lipid disregulation, and hyperglycemia (Nasrallah \& Newcomer, 2004; Newcomer, 2007). Since different antipsychotics have different risk levels, it would seem intuitive that screening for these conditions would be routine for consumers taking antipsychotics in order to find the most appropriate medications for each patient. However, that is not necessarily true. Authors of one study of metabolic screening among those taking antipsychotics showed that less than $30 \%$ of consumers with SMI were screened for weight or metabolic indicators before or during treatment with antipsychotics (Scott et al., 2012). Even when guidelines were issued to reinforce metabolic screening, implementation of these guidelines is often short-lived. Small gains were initially seen in youth receiving screening for metabolic conditions prior to and following prescribing of an antipsychotic shortly after the 2004 American Diabetes Association guidance on the matter was issued, but screening returned to pre-guidance levels within four years (Connolly, Toomey, \& Schneeweiss, 2015).

\section{Integrating Physical and Behavioral Healthcare}

Integrating behavioral and physical healthcare may help improve care for consumers with SMI (Druss, Rohrbaugh, et al., 2001; Frank \& Epstein, 2014), but the degree of coordination or integration has been found to make a difference. Heath and colleagues (2013) described six levels of care, moving from minimal collaboration through fully integrated care (Heath et al., 2013). Traditional models of treating consumers with both physical and behavioral health conditions have relied on crossreferrals between two separate care settings with little collaboration between the two 
(Level 1), or periodic communication about shared consumers only (Level 2; Heath, Wise Romero, \& Reynolds, 2013; Manderscheid \& Kathol, 2014). However, these forms of collaboration depending on two different office sites often prove ineffective because consumers rarely reach the referral site (Manderscheid \& Kathol, 2014). Historical practice patterns and the fact that mental health and primary care providers typically treat different patient populations, the two systems of care have evolved differently over time (Thiejke et al., 2007). However, optimal care depends on the different components of the healthcare delivery system working together toward shared patient outcomes.

Collocated models where physical and mental health practitioners are collocated in the same facility comprised another level of collaborative care within Heath and colleagues' model. Level 3 described basic collaboration in which behavioral and physical health providers saw the same consumers but developed separate treatment plans and had only sporadic discussions about shared consumers (Heath et al., 2013). Level 4 involved collaboration where physical and behavioral healthcare practitioners share clinical practice space and some clinical information was entered in one another's systems (Heath et al., 2013). Collocated care has been identified as one advancement in treating physical and mental health conditions (Powell et al., 2012). Researchers assessing collocated models have typically focused on integration of behavioral health in primary care facilities for consumers with depression and less serious mental health issues, and have shown positive outcomes (Druss, Rohrbaugh, et al., 2001; Pomerantz et al., 2010; Solberg et al., 2013; Thiejke, Vannoy, \& Unutzer, 2007). SMI, however, has rarely been a focus of these studies, nor has Level 4 or 5 care (Gerrity, 2015). 
Integrated care encompassed the final two levels of care described by Heath and colleagues (2013). Level 5 care involved development and implementation of collaborative treatment planning between collocated physical and behavioral healthcare providers. The providers acted as a team and clearly understood one another's roles, but system integration was lacking. Level 6 care fostered collaborative treatment planning for all consumers seen at practice sites with fully integrated systems (Heath et al., 2013). Researchers compiling evidence from other countries have also shown that fully integrated systems allow for better communication among providers and sustained care for consumers (Malm, Ivarsson, \& Allebeck, 2014; Pincus et al., 2015). Research has pointed to effective chronic care working best when supported by multidisciplinary teams with delegation of responsibilities to the appropriate team members, and without barriers between disciplines (Grol, Wensing, Bosch, Hulscher, \& Eccles, 2013; Wagner, Glasgow, et al., 2001).

Three primary factors have led to models of integration within the physical health setting not working optimally, including separate healthcare delivery systems for physical and behavioral health. Each consumer with SMI should have a behavioral health provider and a physical health provider who consults with the behavioral health provider (Manderscheid \& Kathol, 2014). However, that seldom happens in the physical healthcare setting, and is more likely to succeed in the behavioral health clinic (Manderscheid \& Kathol, 2014). The second factor is that separate payment systems exist for behavioral health conditions, and were originally put into place to adequately maximize care (Manderscheid \& Kathol, 2014). Separate financing systems were 
identified by researchers as one of the top barriers to integrating physical and mental healthcare because physicians were not held accountable for all aspects of care (Manderscheid \& Kathol, 2014). The third factor involves stigma related to behavioral health conditions. Stigma is less likely to be present in a behavioral health setting compared to a physical health clinic where mental health issues may not be treated as often, especially SMI (Manderscheid \& Kathol, 2014).

Since many consumers with SMI use behavioral health clinics as their primary care setting, these clinics may be most appropriate for implementing integrated care programs serving consumers with SMI (Kilbourne, Welsh, Mccarthy, Post, \& Blow, 2008; Manderscheid \& Kathol, 2014). There is some evidence that this may be true. Authors of a recent study using Missouri Medicaid data revealed that youth and adult consumers with SMI were more likely to receive guideline-based metabolic screening in CMHCs than in other settings of care (Nicol et al., 2015). However results may have proved more positive for $\mathrm{CMHCs}$ due to a specific $\mathrm{CMHC}$-focused program implemented by Missouri Medicaid prior to the study (Nicol et al., 2015). Use of collaborative approaches to patient care based on the CCM are more common in behavioral health settings in which care managers play a primary role than in physical health settings. As such, care managers help communicate information among members of the treatment team, ensure patients are engaged in treatment, encourage adherence to treatment plans, track outcomes, and monitor progress (Norquist, 2014).

The majority of research conducted assessing collocated primary care within a behavioral health setting have taken place within the VHA system. The VHA is the 
largest of the three administrations that comprise the Veterans Administration system, and addresses physical and mental health of veterans in the United States (Veterans Health Administration, 2015b). The VHA is the largest integrated healthcare system in the country and is comprised of 152 hospitals, 800 outpatient clinics, 126 nursing homes, and 35 domicillaries (Perlin, Kolodner, \& Roswell, 2004; Veterans Health Administration, 2015b). The VHA implemented an EMR system in 1997, which allows for coordination of care across its sites and also assists with research efforts (Perlin et al., 2004). Mental health care within the VHA is focused on recovery and practitioners have implemented coordinated care models and provide evidence-based care (Veterans Health Administration, 2015a).

Several large studies utilizing VHA administrative data, patient surveys, and chart reviews were conducted to assess the effects of colocation of care on receipt of physical health screening tests and guideline-concordant care. Results of these studies were mixed, with improvement in some, but not all, physical health screening tests and fewer patients with mental health issues receiving guideline concordant care than patients without mental health issues (Kilbourne, Welsh, Mccarthy, Post, \& Blow, 2008; Kilbourne et al., 2011). The large sample sizes in these studies allowed for robust findings and pointed to the need for improvement. Similar to the goals of this study, researchers within the VHA system have assessed the impact of collocated care on healthcare service utilization. A randomized controlled trial was conducted to assess differences in service utilization, cost, and health status associated with collocated care. Consumers with SMI $(n=120)$ were randomized to receive physical healthcare either at primary care clinics or 
collocated in mental health clinics. Researchers showed that consumers who were randomized collocated care within the mental health clinics had statistically significant increases in primary care visits, decreases in ED utilization, improvements in physical health status, and a greater likelihood to receive screening tests than consumers randomized to general medical care (Druss, Rohrbaugh, et al., 2001).

More recently, a cohort study was conducted using chart review data from 97 veterans with SMI in the VHA system to assess whether enrollment in a collocated primary care clinic within a behavioral health setting impacted goal attainment for several physical health measures and primary care and ED utilization (Pirraglia et al., 2012). Enrollment in collocated primary care was associated with positive results for attainment of goals in blood pressure (adjusted odds ratio $[\mathrm{AOR}]=2.16 ; 95 \%$ confidence interval [CI], 1.47 - 3.18) low density lipoprotein cholesterol (AOR = 1.60; 95\% CI, 1.10 - 2.34), triglyceride level $(\mathrm{AOR}=1.64 ; 95 \% \mathrm{CI}, 1.06-2.51)$, and body mass index $(\mathrm{AOR}=1.8$, $95 \%$ CI, $1.29,2.54)$. However, enrollment in collocated primary care was not associated with positive results for attainment of high density lipoprotein cholesterol or hemoglobin A1c goals. The number of primary care visits increased significantly from the preenrollment to postenrollment periods (adjusted count $=3.4 ; 95 \% \mathrm{CI}, 2.5-4.8 ; p<$ .001 ), but there was no significant difference in ED service utilization (Pirraglia et al., 2012). While authors of this study found significant differences in several important aspects of care, they did not find significant results for ED service utilization, one of the primary endpoints in the current study (Pirraglia et al., 2012). 
A second cohort study was conducted by researchers within the VHA system to assess the association of patient-aligned care teams (PACT) and utilization of healthcare services by veterans with posttraumatic stress disorder (PTSD; Randall, Mohr, \& Maynard, 2014). PACT was put into place within the VHA to improve care coordination, management of chronic disease, preventive care, and access to medical specialists (Randall et al., 2014). While not identical to the IHC model of care assessed in the current study, some of the goals of treatment were similar. Researchers investigated oneyear pre- and post-PACT periods during the time period of 2009 to 2012 using medical records for 696,379 unique veterans with PTSD. Of those, 336,123 had PTSD in both time periods (Randall et al., 2014). The researchers found that PACT was associated with reduced inpatient admissions (incremental effect $[\mathrm{IE}]=-0.02 ; 95 \% \mathrm{CI},-0.03,-0.01$ ), and use of specialty services $(\mathrm{IE}=-0.45,95 \% \mathrm{CI},-0.07,-0.23)$, and increased primary care visits $(\mathrm{IE}=0.96,95 \% \mathrm{CI}, 0.67,1.25)$. However, PACT was not associated with changes in mental health visits, ED visits, or urgent care visits (Randall et al., 2014). Similarly to the Pirraglia study (2012), Randall and colleagues also did not find significant results for ED service utilization (Randall et al., 2014).

\section{Gaps in Prior Research}

While authors of neither of the VHA studies noted above found significant differences in ED service utilization, there were some important differences between those studies and my study. First, the sample size in the Pirraglia study was small, and therefore the study may not have been powered to observe a difference in this indicator (Pirraglia et al., 2012). The population in the Randall study was different from the 
consumers in my study that it included veterans with PTSD rather than community dwelling consumers with SMI (Randall et al., 2014). While both SMI and PTSD are mental health conditions, the etiologies and treatment guidelines differ. Additionally, the PACT services described above refers to specific care teams within VHA physical health clinics that include mental health practitioners as part of the care team, rather than integrated care within the behavioral health clinic setting (Randall et al., 2014; Veterans Health Administration, 2015a). Furthermore, generalizability of findings of studies in the VHA to other populations may be limited due to advanced use of EMR data in the VHA, as opposed to community settings with less experience. The VHA system also serves a selected group of consumers, who may not be reflective of the general United States population in terms of demographic characteristics including gender, race, or age (Goulet et al., 2007; Pirraglia et al., 2012). Therefore, I addressed integrated care in a community mental health center that likely included consumers who were more reflective of the United States population than those described in the VHA studies.

Integrated care that involves combined physical and mental health treatment plans, rather than simple colocation of services may provide the highest quality of care for consumers with SMI (Gerrity, 2015). However, only a few effective models of integrated care within community behavioral health settings exist for consumers with SMI (Gerrity, 2015; Schaps \& Post, 2015). Researchers from the RAND Corporation recently reviewed 56 programs funded by SAMHSA under the Primary and Behavioral Health Care Integration grant program. Authors of the review described challenges faced by models of physical health collocated in behavioral health settings including culture, 
space, consent to treatment and information sharing, maintenance of medical records, and referral processes. Despite these barriers, one of the programs assessed provided additional medical training for psychiatrists in Massachusetts, and demonstrated a 42\% reduction in ED visits and increases in screening for hypertension and diabetes (Schaps \& Post, 2015). However, this program was not a true example of integrated care because the psychiatrist provided additional medical care, and did not include a medical specialist integrated in the behavioral health setting. Authors also identified one example of a unified system in Tennessee where primary care was collocated in a behavioral health setting at 22 different sites within the Cherokee Health System. In addition to colocation, practitioners in this model used regular meetings to discuss patient treatment plans and integrated EMR systems, much like Level 6 integrated care previously described by Heath et al., 2013. No results were available from this system at the time of publication, and therefore it is still unknown whether integrated healthcare within a community behavioral health setting lead to changes in patient outcomes (Schaps \& Post, 2015). Authors of a recent meta-analysis of interventions for medical conditions among consumers with SMI found low to medium levels of evidence supporting these interventions, and called for further research on implementation strategies in real-world settings (McGinty et al, 2016). I conducted the current study to help fill this need.

\section{Literature Related to Variables}

Consumers with SMI also use intensive healthcare resources at a high rate, specifically inpatient and ED services. Of the 9.6 million adult consumers with SMI in the United States in 2012, almost 63\% used healthcare services (SAMHSA, 2013). In 
2012, there were 269.8 inpatient stays for mood disorders and 121.9 for schizophrenia. These stays were associated with an average length of stay of 6.6 days for mood disorders and 10.4 days for schizophrenia and costs of 4.5 billion and 3.1 billion respectively. Moreover, the all-cause readmission rate within 30 days of hospital discharge was $15 \%$ for mood disorders and $22.4 \%$ for schizophrenia. Mean cost per readmission was $\$ 7,200$ for mood disorders and \$8,600 for schizophrenia (Heslin \& Weiss, 2015). General medical comorbidities and use of acute care services such as EDs were found to be predictors of readmission within 30 days for consumers with schizophrenia as was increased use of outpatient services (Boaz et al., 2013; Vijayaraghavan, Messer, Xu, Sarkin, \& Gilmer, 2015). Furthermore, multiple chronic conditions have been associated with high expenditures among Medicaid and Medicare dual eligible consumers with mental health disorders (Frank \& Epstein, 2014). Patients with co-occurring schizophrenia and multiple medical comorbidities incurred higher monthly all-cause healthcare costs as well as costs related to comorbidities including hypertension, substance abuse, and diabetes (Lafeuille et al., 2014).

Research also points to the contribution of ED to the overall high cost of care among consumers with SMI. Researchers noted that a sharp increase in ED use between 1997and 2007 among Medicaid beneficiaries indicated that the ED may be a safety net care center for persons with poor access to healthcare (Tang, Stein, Hsia, Maselli, \& Gonzales, 2010). Excess use of the ED has been associated with poor access to regular primary care. Canadian researchers showed that lack of a regular general practitioner was associated with higher ED use when controlling for demographic characteristics 
(McCusker et al., 2010). A decline in inpatient psychiatric beds over the past few decades has resulted in consumers with SMI being boarded in EDs waiting for inpatient care or community treatment programs to meet their needs. Researchers have shown that acutely ill consumers with SMI may be held in the ED for as long as 36 hours while waiting for care (Alakeson, Pande, \& Ludwig, 2010; Treatment Advocacy Center, 2015; Zeller, Calma, \& Stone, 2014).

In addition to assessing resource use, I also assessed ACS utilization as part of this study. ACS conditions have been previously defined as those physical health conditions that could be treated in outpatient settings with appropriate access to preventive care (Anderson \& Knickman, 2001). ED visits of this nature have been referred to as ACS conditions, while resulting inpatient admissions for these condition were termed preventable admissions (McCusker et al., 2010; Oster \& Bindman, 2003). Researchers have found that ACS admissions increase as the number of chronic conditions increase (Anderson \& Knickman, 2001), making this an important construct to assess in persons with SMI. The Agency for Healthcare Research and Quality (AHRQ) defined ACS hospitalizations as those with primary discharge diagnoses of diabetes, perforated appendix, COPD, asthma, hypertension, congestive heart failure (CHF), dehydration, bacterial pneumonia, urinary tract infection, angina without procedure, and lower-extremity amputation in people with diabetes (AHRQ, 2015). Researchers identified these conditions through discharge diagnoses using International Classification of Diseases, Ninth Edition, Clinical Modification (ICD-9-CM) codes and procedural codes for surgery. Prior researchers utilizing claims data to identify ACS conditions 
narrowed the original list to include asthma, COPD, CHF, diabetes, and hypertension identified using ICD-9-CM codes only (McCusker et al., 2010; Oster \& Bindman, 2003). For the purposes of this study, the shortened list was used due to data constraints preventing use of the procedural codes defined by AHRQ. ACS ED visits and inpatient hospitalizations were defined using the same set of ICD-9-CM codes as in prior research.

In the United States, Federally qualified health centers (FQHC) provide services to minimize access barriers to care. Researchers assessing FQHCs showed that their use was associated with fewer inpatient admissions and ED visits for ACS conditions in a dual-eligible population (Wright, Potter, \& Trivedi, 2015). FQHCs provide comprehensive services to underserved populations, and as such qualify for enhanced funding from Medicare and Medicaid (United States Department of Health and Human Services, n.d.-b). Since FQHCs provide services that have been shown to reduce barriers to care (Wright et al., 2015), results from this study may help inform a possible role of integrated care for consumers with SMI within the behavioral healthcare setting.

\section{Summary and Conclusions}

SMI affects approximately $5 \%$ of the United States population (Regier et al., 1993; SAMHSA, 2013). Consumers with SMI have shorter lifespans than the general population and higher rates of comorbid physical illness due to lifestyle, side effects from antipsychotic medication, suboptimal screening, and poor care of physical illnesses (De Hert et al., 2011; Mitchell \& Lawrence, 2011; Thornicroft, 2011). Intensive healthcare resource utilization by consumers with SMI and multiple is common, with medical comorbidities and use of acute care services predicting 30-day inpatient readmission rates 
in schizophrenia (Boaz et al., 2013; Heslin \& Weiss, 2015). Researchers also demonstrated that multiple chronic conditions were associated with high spending in Medicaid and Medicare dual eligible beneficiaries with mental health disorders (Frank \& Epstein, 2014).

Integrating behavioral and physical healthcare may help improve care of consumers with SMI (Druss, Rohrbaugh, et al., 2001; Frank \& Epstein, 2014), but the degree of coordination or integration makes a difference. Heath and colleagues (2013) described six levels of care, moving from minimal collaboration defined by simple crossreferrals to fully integrated care defined by creation of joint treatment plans and shared medical records (Heath et al., 2013). Traditional models care for consumers with both physical and behavioral health conditions typically rely on cross-referrals only, and often prove ineffective because consumers often do not attend appointments at the referral sites (Heath et al., 2013; Manderscheid \& Kathol, 2014). Collocated models in which physical and behavioral healthcare practitioners share facility space are an improvement over referral models because providers see the same consumers and have a greater degree of collaboration, but still fall short of ideal (Heath et al., 2013). Researchers assessing these care models have typically focused on integration of behavioral health within primary care facilities to treat depression or other less serious mental health issues. These researchers have shown some positive outcomes in consumers with depression (Druss et al., 2009; Solberg et al., 2013; Thiejke, Vannoy, \& Unutzer, 2007); however SMI has rarely been a focus of colocation studies (Gerrity, 2015). Integrated care encompasses the final two levels of care described by Heath et al., (2013). Level 5 care is characterized by 
collaborative treatment planning, with Level 6 care also including fully integrated electronic medical record systems (Heath et al., 2013).

Since most consumers with SMI utilize behavioral health clinics as their primary care setting, these clinics may be the most appropriate setting for integrated care programs serving consumers with SMI (Manderscheid \& Kathol, 2014). Authors conducting a review of the literature identified several studies that assessed the impact of collocated care in behavioral healthcare settings on access to care, quality of life, and service utilization (Druss, Rohrbaugh, et al., 2001; Kilbourne, Zongshan, Bowersox, Pirraglia, \& Bauer, 2011; Pirraglia, Kilbourne, Lai, Friedmann, \& O’Toole, 2011). These studies, which were described in detail above, were generally conducted within the VHA system and therefore may not be generalizable to populations outside of the VHA. Through this study, I made an original contribution to the research by assessing the impact of a collocated integrated care program with integrated treatment planning on primary care-related service utilization within a community-based setting.

Recent implementation of new health policies and grant programs have advanced the issue through emphasis on quality of care for consumers with SMI and provision of financing structures for integrated care pilots (Barry \& Huskamp, 2011; Druss \& Mauer, 2010; SAMHSA, 2014). The Patient Protection and Affordable Care Act (PPACA) strengthened earlier mental health parity laws and provided additional funding for physical and behavioral healthcare collocation programs (Davis, 2010; Druss \& Mauer, 2010; Golberstein \& Busch, 2013; Mechanic, 2012). SAMHSA has also established the Primary and Behavioral Health Care Integration Program to fund pilot programs 
collocating primary and specialty medical care services in community-based behavioral healthcare settings (SAMHSA 2014). While payers in other countries provide funding mechanisms for integrated care, such mechanisms are lacking in the United States (Pincus et al., 2015). As payers including the Centers for Medicare and Medicaid Services (CMS) move toward quality-based reimbursement rather than quantity-based reimbursement, an understanding of the effectiveness of these programs in reduction of cost and improvement of quality will be important (Burwell, 2015). Through the findings from study, I may help inform future health policy regarding value-based payments.

From a practical perspective, results of this study may help leaders within the data partner CMHC assess the success of the IHC program and identify opportunities for improvement and confounding variables that need to be addressed for optimal implementation. The findings may also enlighten other providers who are interested in implementing integrated physical and behavioral health programs by providing evidence to better inform decisions prior to spending resources during implementation. The most important outcome of this study was the social change implications for consumers with SMI. As integrated care models become more common, assessments of the various care models will improve the level of evidence available to inform optimal care for this population. While consumers with SMI often do not participate in traditional health home models (Lichstein et al., 2014), other models of care that are located within community behavioral healthcare settings may be more appropriate, thus increasing access to physical healthcare and improving outcomes among the SMI population (Barry \& Huskamp, 2011). 
In Chapter 3 I provide an overview of the study methodology, including the research questions and hypotheses, research design and rationale for choosing that design, and a detailed overview of the study data sources. I clearly define the variables and the statistical methods as well as threats to study validity. Ethical considerations are also outlined. 
Chapter 3: Methods

\section{Introduction}

The purpose of this quantitative, retrospective cohort study was to assess the impact of integrated healthcare on overall physical health and ambulatory care sensitive (ACS)-specific inpatient and emergency department (ED) utilization for consumers with serious mental illness (SMI). This was accomplished by evaluating data from an integrated health clinic (IHC) within one large community mental health center (CMHC). The IHC is a primary care clinic located within the CMHC that offers physical health services to adult consumers who receive mental health services at the site. Integrated treatment planning between the physical health and mental health providers is an integral part of the program (CMHC CEO, Personal communication, May 7, 2015). In this chapter, I include a review and rationale for choosing a cohort design, including a discussion of how the design built on prior research and added to the body of literature on integrated care for consumers with SMI. The methods are also discussed in detail, including the population, sample size calculations, and the use of administrative secondary data as the basis for this study. I explore threats to validity resulting from the study design, methods, and choice of data, along with ethical considerations related to the study.

\section{Research Design and Rationale}

In this study, I quantitatively assessed the nature of a predictive relationship, if any, between IHC enrollment and overall physical health and ACS-specific service utilization for consumers with SMI. The independent variable was pre- and post-IHC 
enrollment, and the dependent variables included numbers of overall physical health and ACS-specific inpatient admissions, inpatient days, and ED visits. Covariates included demographic characteristics (age and gender) and disease severity (primary psychiatric diagnosis and comorbidity burden). A retrospective cohort study of secondary administrative healthcare data was used in this quantitative study. Researchers use quantitative research to test theories objectively by assessing numerical relationships among variables, and thus measure the relationship statistically, whereas qualitative analyses are used to understand the meaning that people assign to issues (Yilmaz, 2013). Researchers of quantitative studies use statistical inference to describe numerical changes in measurable characteristics of a population of interest, generalize to other situations, and explain causal relationships (Iversen, 2004; Kraska, 2010). A quantitative research design was chosen because the dependent variables of interest (healthcare resource utilization) were best measured numerically, and assessing the relationship between IHC enrollment and healthcare resource utilization was the aim of the study. Researchers have used quantitative research methods and administrative healthcare data to successfully measure similar outcomes related to healthcare utilization and cost (Birnbaum et al., 1999).

Compared to experimental or other observational designs, the retrospective cohort design was the more appropriate choice because this study was a program evaluation and service utilization and cost available in healthcare administrative data are central to the purpose (Mann, 2003). Cohort studies are typically conducted to determine the incidence or natural history of a condition and retrospective cohort studies use previously collected 
data to perform analyses ad hoc (Mann, 2003). Cohort studies allow for examination of multiple variables and calculation of the effect of each on the outcome of interest, in this case physical health and ACS-specific service utilization (Mann, 2003). An advantage of conducting a retrospective cohort study was that the data were collected for a purpose other than the study at hand, which helped reduce bias. However, the likelihood of data deficiencies exists, as was evident in this study (Mann, 2003).

A prospective experimental design, in which participants would have been randomized to receive IHC, was not appropriate as randomization would not have been ethical and was not as naturalistic (Frankfort-Nachmias \& Nachmias, 2008). Consumers at the CMHC self-selected for inclusion in the IHC, and randomization was therefore not possible (Frankfort-Nachmias \& Nachmias, 2008). If randomization was attempted there may have been ethical concerns regarding one group of consumers receiving physical healthcare and another group being withheld from that care (Frankfort-Nachmias \& Nachmias, 2008). Therefore, choosing a cohort study design using administrative claims and EMR data was more appropriate for this study. A time-series design may also have been an appropriate approach because this design would have allowed for consumers with SMI to be evaluated in their natural treatment settings (Frankfort-Nachmias \& Nachmias, 2008). However, this study design was not chosen due to time constraints and restrictions of Walden University to implement an intervention.

Secondary data refers to data previously collected by others for purposes not related to the current research study (Frankfort-Nachmias \& Nachmias, 2008). Administrative healthcare data that have been collected by the CMHC for purposes of 
patient management and billing were used since the purpose of the study was to evaluate existing care strategies employed by the CMHC. Administrative healthcare data have been used in outcomes research for decades with success in analyzing certain types of outcomes that can be measured with these data sources, including healthcare utilization and cost (Birnbaum et al., 1999). Linking administrative data to other sources of clinical information such as EMR data may help provide additional useful information about the quality of patient care (Birnbaum et al., 1999), and I applied this method to my study.

Several researchers have assessed integrated care programs in the United States using cohort studies and nonprobability-sampling methods due to the scarcity of these programs, with some using data from the Veteran's Health Administration (VHA) system (Kilbourne et al., 2011; Pirraglia et al., 2011), and others studying small accountable care organizations or patient-centered medical homes that have integrated care pilot programs (Mueser, Bartels, Santos, Pratt, \& Riera, 2012b; Reiss-Brennan, 2014). However, larger scale studies of integrated care programs are not possible due to the fact that nation-wide sources of data such as administrative claims databases do not capture sufficient information to identify which patients are part of integrated care models; therefore smaller datasets or chart reviews that identify integrated care program participation provide the best opportunity for analysis (Gerrity, 2015).

The work of two previous groups of researchers within the VHA system helped inform the study design and endpoints of the current study (Pirraglia et al., 2012; Randall, Mohr, \& Maynard, 2014). Pirraglia et al. (2012) assessed 97 veterans with SMI enrolled in a collocated integrated primary care clinic within a VHA behavioral health outpatient 
center and assessed primary care clinic enrollment on outpatient and ED service utilization as well as physical health goal attainment. They analyzed change in these parameters between the 6-months before and 6-months after enrollment in the primary care clinic and found that clinic enrollment was associated with attainment of some cardiovascular risk goals and an increase in primary care visits. While Pirraglia and colleagues (2012) also employed a cohort design, there were several important differences between their study and my study reported here. Pirraglia et al. used chart review data from the VHA system as the primary source of data, and thus were able to collect physical health measures such as body mass index, hemoglobin A1c (HbA1c) level, and lipid test results that were not available to me to assess due to data limitations. Furthermore, I assessed inpatient admissions and length of inpatient stay, which were not assessed by Pirraglia and colleagues, although primary care outpatient visits were not captured in the current study.

Randall, Mohr, and Maynard (2014) also used a cohort study design to assess the association of the patient-aligned care teams (PACT) model of care to use of healthcare services among veterans with post-traumatic stress disorder (PTSD). One-year pre- and post-PACT periods were assessed and the authors indicated that PACT was associated with a lower rates of hospitalization and specialty service, with an increase in primary care visits (Randall et al., 2014). Unlike the current study, the pre- and post-PACT periods were not assessed by veteran, but rather pre- and post- a particular point in time (2010) when PACT was implemented within the VHA system. Therefore, sample sizes differed in the pre- and post-PACT periods (Randall et al., 2014). Randall et al. (2014) 
assessed healthcare service utilization using VHA administrative data; however, data for all levels of care were available, while only inpatient admissions and ED visit data were available for the current study (Randall et al., 2014).

\section{Methodology}

An overview of the methodology will be provided in this section, including the population and sampling procedures, the use of archival data, and the specific data sources used for this study, with an explanation of considerations to be taken into account and permissions necessary to obtain the data. In the latter half of the chapter, I will address the variables and how they are operationalized.

\section{Population}

Approximately $5 \%$ of the United States population has a diagnosis of SMI (SAHMSA, 2013), including 1\% with schizophrenia, 2\% with bipolar disorder, and 6\% with major depressive disorder (Regier et al., 1993; SAHMSA, 2013). The CMHC system providing the data for this study served approximately12,600 consumers in 2013 in nine locations throughout the region, with about 3,500 consumers served at the primary site, from which data were obtained (CMHC CEO, Personal communication, May 7, 2015). It was estimated that approximately 1,000 consumers would the IHC during the study period (CMHC CEO, Personal communication, May 7, 2015), and approximately 1,500 actually did. Therefore the target population consisted of all CMHC consumers who enrolled in the IHC. 


\section{Sampling and Sampling Procedures}

Since this was an analysis of secondary data, the IHC cohort was extracted from the parent population (all consumers at the CMHC accessing IHC services) based upon operational definitions and inclusion criteria (purposeful convenience sample). The sample for this program evaluation was a nonprobability purposeful convenience sample. Nonprobability sampling refers to samples in which there is no way to specify the probability of each consumer's inclusion in the sample or assure that each consumer has a chance for inclusion, and a convenience sample is chosen from those available for measurement (Frankfort-Nachmias \& Nachmias, 2008). This was the most appropriate method for this study because the study evaluated integrated health program at a specific CMHC. Although nonprobability sampling is not as robust as probability sampling (Trochim, 2006), a convenience sample was necessary for this study because of the nature of the study. Furthermore, there are no publically available data sets that contain information on integrated care programs. Convenience sampling was the most efficient way to access this population and answer the questions posed for this study.

The sample included all consumers who enrolled in the IHC between October 1, 2013 and September 30, 2015. Patient identification numbers from the IHC electronic medical record (EMR) data file were used to identify these consumers. Inpatient and ED authorization data were sent to the CMHC by two payers beginning in October 2013. In order to maximize available data for consumers enrolled in the IHC, the latest possible date to receive files was chosen. Data for consumers whose first visit with the IHC 
occurred after September 30, 2015 were not analyzed, as a full six months of data following initial contact was required.

The alpha level (or p-value) is the chance of error that researchers are willing to accept (Walden University, 2014). For the statistical tests, a p-value less than 0.05 was used as the cutoff for statistical significance, with a confidence interval of $95 \%$. A pvalue of less than 0.05 indicates that one in twenty results would be by chance, while the confidence interval of $95 \%$ indicates that 95 out of 100 times the observed score will fall within the range of values (Greenhalgh, 2014). Effect size identifies the strength of the conclusions about group differences or the relationships among variables. An effect size of 0.1 is considered a small effect size and was chosen to calculate sample size for the regression analyses (Cohen, 1992). Power refers to the likelihood of finding a statistical difference when one exists and was set at $80 \%$ (Field, 2013).

Using $\mathrm{G}^{*}$ Power to compute sample size, estimates were derived for multiple linear regression, multiple logistic regression, and paired sample t-tests to arrive at the appropriate sample size. The sample size calculation for multiple linear regression with an alpha of 0.05 , effect size of 0.1 , power of 0.80 , and 4 predictors yielded a sample size of 126 (Faul, Erdfelder, Lang, \& Buchner, 2007). Calculations the other tests yielded lower sample sizes. The calculations for the t-test yielded a sample of 102, with 51 in each cohort. The calculation for multiple logistic regression, one sided, odds ratio of 3.86 , $\operatorname{Pr}(\mathrm{Y}=1 \mid \mathrm{X}=1) \mathrm{H} 0=0.1$, with a $p$-value of 0.05 , power of $0.80, R^{2}$ other $\mathrm{X}$ of 0.0625 , and $\mathrm{X}$ parm $\pi$ of 0.5 , and Poisson distribution yielded a sample size of 49 and for a binomial distribution, a sample size of 108. The calculation for a Poisson regression considered the 
following input parameters: one tail, $\operatorname{Exp}(B 1)$ of $0.8, \mathrm{p}$-value of 0.05 and power of 0.80 , base rate of 0.04 , mean exposure of $180, R^{2}$ other $\mathrm{X}$ of 0.0625 , binomial distribution and X parm $\pi$ of 0.5 , and yielded a sample size of 83 (Faul et al., 2007). Therefore the larger sample size calculation of 126 for the multiple linear regression analysis was relied upon for the necessary sample size of this study.

Data for the entire population of consumers enrolled in the IHC who were 18 years and older, and for whom a full six months of data were available pre- and postenrollment was analyzed, resulting in a sample size of 370 , greater than the minimum sample size required. There were several reasons for using the larger sample for the study cohort rather than selecting the minimum number necessary. First, increasing the sample size helped control for Type II error, even if just increasing the sample by $10 \%$ (Frankfort-Nachmias \& Nachmias, 2008). Furthermore, a larger sample size helped control for attrition in the sample (Frankfort-Nachmias \& Nachmias, 2008). There could have been a great deal of attrition in a sample of consumers with SMI, due to the transient nature of this population, and a full six months of data were necessary pre- and post-IHC enrollment. In this study, the difference between the sample size estimate and the population expected in the IHC was large enough to account for attrition.

\section{Archival Data}

The IHC is a primary health clinic that offers physical health care to adult consumers who are enrolled in mental health services at the data partner CMHC. Consumers seeking mental health services were referred to the IHC by their mental health treatment team. Consumers could choose whether or not to access IHC services. 
The mental health and physical health providers engaged in integrated treatment planning for those consumers who chose to seek care with the IHC. Clinical data were stored in EMRs for mental health and IHC services; however, the EMRs were separate, nonintegrated systems (CMHC CEO, Personal communication, May 7, 2015).

Several data files were obtained from the data partner CMHC, including:

1. CMHC contact files containing consumer demographic information from October 1, 2013 to December 31, 2015 for all consumers of the CMHC

2. Inpatient admission and ED visit authorization data from October 1, 2013 to December 31, 2015 for consumers of the CMHC enrolled in either AmeriGroup or UnitedHealth health plans

3. IHC EMR data from October 1, 2013 to December 31, 2015 for consumers seeking care at the IHC

Contact files were available for all consumers of the $\mathrm{CMHC}$, and contained basic demographic information (e.g. age, gender, insurance provider) and primary psychiatric diagnosis, as well as a patient identification number, which enabled linking of the other sources of data at a patient level. This file also contained the first date of service at the CMHC for each consumer, which was used to ensure a full six months of data pre- and postenrollment in the IHC. Two of the three major health plans sent authorization files for all CMHC consumers seen at an ED or admitted to an inpatient setting. AmeriGroup sent these files weekly while UnitedHealth sent them monthly. These data sets included the patient identifier, date of the admission/visit, the number of days of the authorization for inpatient admissions, and the International Classification of Diseases, Ninth Edition, 
Clinical Modification (ICD-9-CM) code corresponding to the primary reason for the inpatient admission or ED visit. EMR data were available for the consumers enrolled in the IHC program, and contained the patient identifier and date of the visit to the IHC. The IHC began accepting consumers in July 2013; however, utilization data were not available for the IHC program until October 1, 2013.

Data from all three of these secondary data sources were extracted from the CMHC's information systems by a CMHC data analyst and transferred to me in Microsoft Excel format by the Chief Operations Officer using a secure file transfer protocol (SFTP). The data were not de-identified, and were stored on an encrypted hard drive until de-identification. The original files were destroyed, leaving only the deidentified data files.

Data considerations. Several considerations regarding the above-mentioned data files should be noted. First, not all consumers with entries in the CMHC contact files or authorization data participated in the IHC program. Therefore, those two data files were matched against the IHC EMR file using patient identification number to limit the analysis set to only those consumers who had used IHC services.

Second, the majority of CMHC consumers were served by three health plans but only two of the three insurance carriers provided authorization data files for inpatient and ED visits back to the CMHC (AmeriGroup and UnitedHealth). Therefore the analyses were limited to the consumers covered under these two health plans, which accounted for approximately $67 \%$ of the CMHC's population (CMHC CEO, Personal communication, May 7, 2015). These data files were de-duplicated to ensure that visits were not being 
double-counted. For the inpatient data, there was a need to combine separate authorizations for the same stay to reflect a single inpatient stay. The earliest admission date and latest discharge date captured were used to accurately calculate number of inpatient days for each admission. To ensure accuracy in merging separate authorizations, rules were applied to ensure each authorization included the same patient identification number, authorization number, initial admission date, and facility to correctly define a single episode of care (Birnbaum et al., 1999).

Data regarding physical health outpatient visits provided outside of the IHC were not reported back to the CMHC or available in the data files obtained for this study. Consumers referred to the IHC were unlikely to have a different primary care provider, but that was a possibility. This lack of outpatient data was noted as a potential confounder and a limitation to the study.

Data acquisition and permissions. Permission to access and analyze the data used in this study was obtained from the Chief Executive Officer and Chief Operations Officer of the CMHC. In June 2015, a Business Associate Agreement (BA) was executed between me and the data partner CMHC (Appendix A), which was required by the CMHC to ensure compliance as a Covered Entity under the Health Insurance Portability and Accountability Act (HIPAA) of 1996 (United States Department of Health and Human Services, n.d.-a). A letter of Cooperation (Appendix B), required by Walden University, was also executed, further detailing how I used the data.

Following execution of the Walden University Confidentiality Agreement (Appendix C) and Data Use Agreement (Appendix D) the data files were made available 
to me by the CMHC using a SFTP, and data were stored on an encrypted hard drive. The data were not de-identified upon delivery; therefore, all original data was stripped of personally identifiable information and files were created for analysis, using the patient identification number as the common link between files. The original files were destroyed following conversion to the analysis files so that no files containing personally identifiable health information remained on my encrypted hard drive.

\section{Variable Operationalization}

Independent variable. The independent variable was enrollment in the IHC program. Enrollment was an identified field in the database, coded as " 0 ” for preenrollment in the IHC and " 1 " for postenrollment in the IHC.

Dependent variables. The dependent variables included: (a) number of inpatient admissions; (b) number of inpatient days; and (c) number of ED visits where the primary diagnosis was for a physical health condition. Each inpatient admission and ED visit was counted separately as one single event and the total number of admissions/visits were calculated for each consumer for the 6-month time periods before and after enrollment into the IHC. Similarly, the number of inpatient days were counted for each admission and summed to arrive at an absolute number of days hospitalized per consumer for the pre- and postenrollment timeframes. Means, standard deviations, medians and interquartile ranges for each dependent variable were calculated for the period before and after IHC enrollment. The percentage of consumers experiencing an inpatient admission or ED visit in both the pre- and postenrollment periods was also assessed. 
Classification of dependent variables. Each inpatient and ED visit authorization contained a single ICD-9-CM code corresponding to the primary reason for the admission or visit. These ICD-9-CM codes were used to classify whether the admission or visit was for a physical health or mental health diagnosis, and only physical health diagnoses qualified for this analysis. A further determination was made as to whether the inpatient admission or ED visit was an ACS-specific condition, as defined by prior research.

Generally, ACS conditions are those that could have been treated in an outpatient setting if appropriate access were available (Anderson \& Knickman, 2001). ED visits of this nature are typically referred to as ACS conditions, while a resulting inpatient admission for the condition would be considered a preventable admission (McCusker et al., 2010; Oster \& Bindman, 2003). For the purposes of this study, both inpatient admissions and ED visits were referred to as ACS, and are defined in Table 1 using the ICD-9-CM codes from prior research.

Table 1

ACS Conditions

\begin{tabular}{ll} 
Condition & ICD-9-CM Codes \\
\hline Asthma & $493,493.0,493.01,493.1,493.2,493.9$ \\
Chronic obstructive & $466,491,491.1,491.20,491.21,491.8,492,492.0$, \\
pulmonary disease & $492.8,494,496$ \\
Congestive heart failure & $402.01,402.11,402.91,428,428.0,428.1,428.9,518.4$ \\
Diabetes & $250.0-250.3,250.8-250.10,250.12,250.13,250.20$, \\
& $250.22,250.23,250.30,250.32,250.33,250.90,250.92$, \\
Hypertension & 250.93 \\
& $401.0,401.9,402.00,402.10,402.90,403.0,404.0$, \\
& $405.0,437.2$ \\
\hline
\end{tabular}


Covariates included the demographic characteristics of consumer age and gender and markers of disease severity including primary psychiatric diagnosis and comorbidity burden. Primary psychiatric diagnosis was categorized according to prior research (Ettner, Frank, McGuire, Newhouse, \& Notman, 1998; Ettner, Frank, Mark, \& Smith, 2000) as: psychotic disorder, mood disorder, or substance abuse disorder in combination with another psychiatric diagnosis. Comorbidity burden was calculated according to the Charlson Comorbidity Index (CCI), which will be described in greater detail below.

Table 2

Coding of Variables

\begin{tabular}{lll}
\hline Variable & $\begin{array}{c}\text { Type of } \\
\text { Variable }\end{array}$ & Coding \\
\hline $\begin{array}{l}\text { Independent variable } \\
\text { Enrollment in IHC }\end{array}$ & Binary & $0=$ preenrollment $; 1=$ postenrollment
\end{tabular}

Dependent variables

Absolute number of ED visits

Absolute number of inpatient admissions

Absolute number of inpatient days

ED visit type

Inpatient visit type

Covariates

Age

Gender

Initial psychiatric diagnosis
Continuous Absolute number of ED visits in each study period

Continuous Absolute number of inpatient admissions in each study period

Continuous Sum of days in each study period

Nominal $\quad 0=$ mental health; $1=$ physical health non-ACS; 2 = physical health ACS

Nominal $\quad 0=$ mental health; $1=$ physical health non-ACS; 2 = physical health ACS

Continuous Reported as a whole number and calculated by subtracting date of birth from index date

Nominal $\quad 0=$ female; $1=$ male

Nominal $1=$ psychotic disorders (schizophrenia, schizoaffective disorder, bipolar disorder); $2=$ mood disorders such as 
depression or anxiety; and $3=$ substance abuse in conjunction with another psychiatric disorder

CCI comorbidity burden

Ordinal

$0=$ no comorbidity $1=$ mild comorbidity; 2 = moderate comorbidity; 3 or more $=$ severe comorbidity

\section{Data Analysis Plan}

All data were stored in an SPSS data file for analysis and analyzed using SPSS Statistics for Mac, Student Edition, version 23 (IBM). In addition to the data considerations noted previously in this chapter, several data cleaning procedures were also undertaken. First, outliers in the data were identified, defined as those data points more than three standard deviations outside of the mean. Outlier values in data can lead to both type 1 and type 2 errors (Field, 2013; Laureate Education (Producer), 2009). Rather than deleting the outlier data, data were transformed by calculating the square root, as recommended for positively skewed data (Laerd Statistics, 2015b). Normality of data was also problematic with these data, so Wilcoxon signed-rank tests, the nonarametric equivalent statistics, were run in place of paired-samples t-tests (Laureate Education (Producer), 2009). Rather than deleting consumers with missing data, missing data were treated as a separate category (Laureate Education (Producer), 2009).

Research Question and Hypotheses. The following is a summary of the two main research questions and associated hypotheses of this study.

RQ1: What is the predictive relationship, if any, between IHC enrollment and overall physical health service utilization for consumers with SMI when controlling for demographic characteristics and disease severity? 
H01: There is no statistically significant predictive relationship between IHC enrollment and overall physical health service utilization (number of inpatient admissions and ED visits, number of inpatient days) when controlling for demographic characteristics (age and gender) and disease severity (primary psychiatric diagnosis, disease burden).

$\boldsymbol{H A 1 :}$ There is a statistically significant predictive relationship between IHC enrollment and overall physical health service utilization (number of inpatient admissions and ED visits, number of inpatient days) when controlling for demographic characteristics (age and gender) and disease severity (primary psychiatric diagnosis, disease burden).

RQ2: What is the predictive relationship, if any, between IHC enrollment and ACS-specific service utilization for consumers with SMI when controlling for demographic characteristics and disease burden?

H02: There is no statistically significant predictive relationship between IHC enrollment and ACS-specific service utilization (number of inpatient admissions and ED visits, number of inpatient days) when controlling for demographic characteristics (age and gender) and disease severity (primary psychiatric diagnosis, disease burden).

$\boldsymbol{H A 2 :}$ There is a statistically significant predictive relationship between IHC enrollment and ACS-specific service utilization (number of inpatient admissions and ED visits, number of inpatient days) when controlling for demographic characteristics (age and gender) and disease severity (primary psychiatric diagnosis, disease burden).

Risk Adjustment. Risk adjustment is a corrective tool used to equalize cohorts in 
statistical analysis by adjusting for the differences in risk among specific patients (Society of Thoracic Surgeons, 2015). Risk adjustment has often been used to adjust payment under capitated insurance models to adjust for disease severity in a given population (Ettner et al., 1998; Ettner et al., 2000). Primary diagnosis has been used as a risk adjustment method in past studies, as have diagnosis related groups and demographic characteristics (Ettner et al., 2000; Newhouse, Buntin, \& Chapman, 1997). Researchers have shown that models using only demographic characteristics perform poorly, but when adding psychiatric diagnosis the models performed much better (Ettner et al., 1998). For the purposes of this analysis, risk adjustment included type of primary psychiatric diagnosis in the pre-IHC enrollment period, as defined by ICD-9-CM codes. Psychiatric diagnosis was divided into three categories, based on prior research: (a) psychotic disorders (schizophrenia, schizoaffective disorder, bipolar disorder); (b) mood disorders such as depression or anxiety; and (c) substance abuse in conjunction with another psychiatric disorder (Ettner et al., 1998; Ettner et al., 2000).

The CCI was also used to risk adjust in this analysis. The CCI identified 17 comorbid conditions predictive of mortality, and was used to assess overall comorbidity in the six months prior to enrolling in the IHC (Table 3). The CCI has been used extensively as a risk adjuster in database research, and has been shown to be predictive of mortality and future excess costs (Charlson, Wells, Ullman, King, \& Shmukler, 2014; D’Hoore, Bouckaert, \& Tilquin, 1996). Moreover, about half of the excess mortality among consumers with schizophrenia and bipolar disorder are attributable to diseases included in the CCI (Laursen, Munk-Olsen, \& Gasse, 2011), indicating that it is an 
appropriate index for risk adjustment in this study. Deyo, Cherkin, and Choi (1992)

adapted the CCI for use with ICD-9-CM codes (Deyo, Cherkin, \& Ciol, 1992; Quan et al., 2005). The CCI is calculated as a weighted measure, with each condition weighted according to its relative risk of mortality, and can be age-adjusted by adding a point for each decade over the age of 40 (Yang, Chen, Hsu, Chang, \& Lee, 2015). Frequency of the identified ICD-9-CM codes on inpatient or ED authorizations in the six months prior to IHC initiation was used to calculate the age-adjusted CCI for each consumer.

Table 3

Charlson Comorbidity Index Coding and Weighting

\begin{tabular}{|c|c|c|}
\hline Comorbidity & ICD-9-CM Codes & Weight \\
\hline Myocardial infarction & $410 . x, 412 x$ & 1 \\
\hline Congestive heart failure & $428 . x$ & 1 \\
\hline Peripheral vascular disease & $\begin{array}{l}443.9,441 . x, 785.4, \mathrm{~V} 43.4, \text { procedure } \\
38.48\end{array}$ & 1 \\
\hline Cerebrovascular disease & $430 . x-438 . x$ & 1 \\
\hline Dementia & $290 . x$ & 1 \\
\hline Chronic pulmonary disease & 490.x-496.x, 500.x-505.x, 506.4 & 1 \\
\hline Rheumatic disease & $\begin{array}{l}\text { 710.0, 710.1, 710.4, 714.0-714.2, } \\
714.81,725 . \mathrm{x}\end{array}$ & 1 \\
\hline Peptic ulcer disease & $531 . x-534 . x$ & 1 \\
\hline Mild liver disease & $571.2,571.4-571.6$ & 1 \\
\hline $\begin{array}{l}\text { Diabetes without } \\
\text { complications }\end{array}$ & $250.0-250.3,250.7$ & 1 \\
\hline $\begin{array}{l}\text { Diabetes with chronic } \\
\text { complication }\end{array}$ & $250.4-250.6$ & 2 \\
\hline Hemiplegia or paraplegia & 344.1, 342.x & 2 \\
\hline Renal disease & 582.x, 583-583.7, 585.x, 586.x, 588.x & 2 \\
\hline $\begin{array}{l}\text { Any malignancy, including } \\
\text { lymphoma and leukemia } \\
\text { (excluding malignant } \\
\text { neoplasm of skin) }\end{array}$ & 140.x-172.x, 174.x-195.8, 200.x-208.x & 2 \\
\hline $\begin{array}{l}\text { Moderate or severe liver } \\
\text { disease }\end{array}$ & $456.0-456.21,572.2-572.8$ & 3 \\
\hline Metastatic solid tumor & $196 . x-199.1$ & 6 \\
\hline
\end{tabular}


Statistical procedures. Overall physical health and ACS-specific inpatient admissions and ED visits were assessed comparing the six months before versus the six months following the initial IHC enrollment date. Although paired-sample $t$-tests to determine differences in means of service utilization outcomes between the pre- and postenrollment timeframe for consumers in the IHC were originally planned, Wilcoxon signed-rank tests were used instead due to the skewed nature of the data. Logistic regression, a method for modeling a categorical response variable and either categorical or continuous predictor variables (Bewick, Cheek, \& Ball, 2005; Field, 2013), were run to examine the odds of consumers experiencing any inpatient admission or ED visit before and after IHC enrollment. Enrollment was examined first as the sole fixed effect. Age, gender, initial psychiatric diagnosis, and CCI score were added to the fixed-effects portion of the model. Four separate logistic regression models assessed overall physical health service utilization and ACS-specific service utilization for inpatient admissions and ED visits.

Linear regression is a technique used by researchers to assess the predictive relationship between variables. Simple linear regression involves an outcome variable and one predictor variable, while multiple regression involves multiple predictor variables (Field, 2013). Simple linear regression models were planned to examine the predictive relationship between physical health service utilization (both overall and ACSspecific) and IHC enrollment alone. Multiple linear regression analyses using the forced entry method examined the predictive relationship between physical health service 
utilization and IHC enrollment, demographic characteristics, and disease severity. Consistent with the forced entry method, predictors were entered into the model at the same time, making no decisions regarding the order that the predictors were added (Field, 2013). Separate multiple regression models were run for overall physical health service utilization and ACS-specific service utilization for each type of service, including inpatient admissions, inpatient days, and ED visits, resulting in a total of six multiple linear regression models.

Poisson regression models are useful when outcome data are counts, as in this current study (Field, 2013; Shanmugam, 2007) and also for low frequency, highlyskewed data (Nussbaum, Elsadat, \& Khago, 2008). The data in the current study was highly skewed, and therefore, Poisson regression analyses were employed to examine the predictive relationship between IHC enrollment and physical health service utilization. Enrollment was examined first as the sole fixed effect, with age, gender, primary psychiatric diagnosis, and CCI score added to the fixed-effects portion of the models. Separate Poisson regression models were run for overall physical health service utilization and ACS-specific service utilization, and for each type of service, including inpatient admissions, inpatient days, and ED visits, resulting in a total of six Poisson regression models.

Interpretation of results. For the statistical tests, a p-value $>0.05$ was used to as the cutoff for statistical significance, with a confidence interval of $95 \%$. A p-value of $>0.05$ indicates that one in 20 results would be by chance, while the confidence interval of $95 \%$ indicates that 95 out of 100 times the observed score will fall within the range of 
values (Greenhalgh, 2014). Paired samples t-tests were planned to assess whether the means of inpatient admissions, days hospitalized, and ED visits were statistically significantly different pre- and post- enrollment into the IHC. However Wilcoxon signedrank tests were run instead as the non-parametric alternative and assessed using the same significance levels as the paired samples t-tests would have been.

Logistic regression results were interpreted using odds ratios, which are the odds of an event occurring in one group versus another (Field, 2013). Odds ratios of less than one indicate a negative relationship between the independent variable and the dependent variable whereas those greater than one indicated a positive relationship (Morrow, 2014). In this study, odds ratio assessed the odds of any inpatient admission or ED visits occurring in the pre- and post-enrollment timeframe. Multiple linear regression results were interpreted using the $F$-test to assess whether the overall models were statistically significant and predictive of service utilization, while the $t$-statistics for each predictor variable indicated the level of its statistical significance.

\section{Threats to Validity}

The primary limitation of the study was associated with the methodology. Since a retrospective cohort design using a convenience sample, bias is introduced in that the consumers enrolled in the IHC of their own volition. Therefore, the results were not generalizable to a population beyond the clients at the $\mathrm{CMHC}$ who enrolled in the IHC program (Mann, 2003; Phua, 2007). However, this study will add to the body of literature about collocated integrated care programs in general. Confounding variables are another threat to the study. Confounding variables are independently related to both the 
independent and dependent variables (Mann, 2003). In this study, regression analyses were run to help control for demographic characteristics that may confound the study. However, other confounding variables not captured in the data available for analysis may have impacted the results, and this issue was addressed in the limitations section of Chapter 5.

Furthermore, this study used secondary data (administrative claims and EMR data) to assess whether the IHC was associated with physical health inpatient and ED service utilization. The information that secondary data provided was more limited than what I would have collected if I gathered my own data (Frankfort-Nachmias \& Nachmias, 2008). There may have been other important indicators of IHC success, such as consumer satisfaction, that were not assessed using administrative secondary data. However, using administrative data for this study may allow others to more closely replicate the methodology with other integrated care programs (Frankfort-Nachmias \& Nachmias, 2008). There may also have been confounding variables not accounted for in the administrative data that influenced change in resource use. Due to these threats to internal validity, causality was not confirmed. Finally, different persons involved in the consumer's care entered data used in the analysis into the administrative systems. Therefore, data entry errors may have existed leading to inaccuracies in the data. However, use of both authorization and EMR data sources to identify inpatient admissions and ED visits increased the validity of the findings through angulation (Frankfort-Nachmias \& Nachmias, 2008). 
I initially thought that study sample retention would pose a threat to internal validity and attrition would need to be accounted for (Frankfort-Nachmias \& Nachmias, 2008). However, this did not turn out to be the case with the consumers at the CMHC. One reason may be that they were covered by Medicaid and not as likely as consumers covered by commercial insurance to switch health plans or move to other locations. Experimental mortality may have been an issue, and consumers in the IHC cohort may have been lost to attrition following IHC initiation, but all data prior to initiation were available since I limited the analysis to those consumers with a full six months of data pre- and post- IHC enrollment (Frankfort-Nachmias \& Nachmias, 2008).

\section{Data Considerations and Ethical Procedures}

There were several ethical considerations for this study; most relating to the fact that the research data included protected health information (PHI). The Health Information Portability and Authorization Act (HIPAA) provided federal protections for individually identifiable health information and gives patients rights with respect to use of that information. However, HIPAA is balanced to allow disclosure of PHI needed for patient care and other important purposes (United States Department of Health and Human Services, n.d.-a). Several documents regarding researcher access to the data were put into place prior to data transfer. In June 2015, I signed a Business Associate agreement with $\mathrm{MHC}$, which is required by $\mathrm{MHC}$, as it is a Covered Entity as defined by HIPAA (Appendix A). Before receiving data from the CMHC, a Confidentiality Agreement acknowledging researcher access to confidential information was signed, holding me accountable to not disclosing the confidential information, either formally or 
informally (Appendix C). Furthermore, both representatives from the CMHC and I signed a Data Use Agreement (DUA) that identified the exact data to be transferred and indicated the purposes for which the data were used (Appendix D).

HIPAA guidelines also dictate that data collection and storage must be secure to protect PHI of research subjects. To comply with HIPAA regulations, the data files were made available by the CMHC using a secure file transfer protocol, and data were stored on an encrypted hard drive. The data were not de-identified upon delivery; therefore, all original data was stripped of personally identifiable information and files were created for analysis, using the patient identification number as the primary patient identifier. The original files were destroyed following conversion to the analysis files so that no files containing PHI remained on an encrypted hard drive, preventing data from being linked back to an individual consumer of services (Frankfort-Nachmias \& Nachmias, 2008).

There were several other ethical considerations for this study. The consumers of the CMHC all have an SMI diagnosis, and therefore are considered a vulnerable population. No intervention was conducted as part of the study, as the IHC program had already been implemented by the CMHC. The lack of intervention with human participants minimized the risks of dealing with a vulnerable population. A quasiexperimental design was chosen because this design allows for consumers with SMI to be evaluated in their natural treatment settings. Utilization of IHC and the associated outcomes, therefore, are naturally occurring in the CMHC setting.

The data sources for this study were secondary data sources, so no informed consent was required. However, caution was used when analyzing the data and reporting 
results. Due to the sensitivity of healthcare related information, I protected the privacy of the information (Frankfort-Nachmias \& Nachmias, 2008). In addition to the storage of data described above, results were reported at the population level only for dissemination purposes; therefore data cannot be directly linked to an identified consumers, further protecting privacy (Frankfort-Nachmias \& Nachmias, 2008). The Walden University Institutional Review Board (IRB) reviewed and approved the study prior to inception (IRB approval number 05-17-16-0449950).

\section{Summary}

As discussed in this chapter, the purpose of this study was to assess the association of integrated care with physical health inpatient and ED utilization for consumers with SMI through an evaluation of an integrated health clinic within one large CMHC. The main research question addressed by this study was: What is the relationship between IHC enrollment and physical health service utilization, including ACS-specific conditions, for consumers with SMI when controlling for demographic characteristics? The research question was answered using a retrospective cohort analysis of administrative healthcare data.

The independent variable was enrollment in the IHC and the dependent variables included the absolute numbers of inpatient admissions and ED visits and absolute number of inpatient days where the primary diagnosis was for a physical health condition. In this study, I used a retrospective cohort design, in which administrative healthcare data for CMHC consumers was obtained for the period starting October 1, 2013 and ending December 31, 2015. Physical health inpatient admissions and ED visits (overall and 
ACS-specific) were compared for the six months before versus six months following initial IHC enrollment date. In Chapter 4, I present the results of the study, including a detailed description of the statistical findings and associated results tables. 
Chapter 4: Results

\section{Introduction}

In this quantitative study, I assessed the impact of integrated care on overall physical health and ambulatory care sensitive (ACS)-specific inpatient and emergency department (ED) utilization for participants with serious mental illness (SMI). The study evaluated the effect of an integrated health clinic (IHC) providing physical health care within a large community mental health center $(\mathrm{CMHC})$. The first research question that was addressed by this study was: What is the predictive relationship, if any, between IHC enrollment and physical health service utilization for participants with SMI when controlling for demographic characteristics and disease severity? The second research question sought to assess the predictive relationship, if any, between IHC enrollment and ACS-specific service utilization for participants with SMI when controlling for demographic characteristics and disease severity.

This chapter includes (a) secondary data used in the analysis, including the timeframes of the data and the final sample size, (b) baseline demographic information characterizing the sample and results from the univariate analyses, including a detailed description of risk stratification data, (c) findings from logistic, multiple linear, and Poisson regression analyses, first for the analyses of overall physical health utilization, and next for ACS-specific utilization, and (d) findings from additional analyses. 


\section{Data Collection}

Data were collected from three sources within the CMHC, including CMHC contact files containing consumer demographic information from October 1, 2013 to December 31, 2015 for all consumers of the CMHC, inpatient admission and ED visit authorization data from October 1, 2013 to December 31, 2015 for consumers of the CMHC enrolled in either AmeriGroup or UnitedHealth health plans, and IHC electronic medical record (EMR) data from October 1, 2013 to December 31, 2015 for consumers seeking care at the IHC. A total of 1507 potential participants had at least one visit to the IHC. Of those, 370 met the enrollment criteria, including having six months of data preand post- index date (first visit to the IHC) available for analysis, were 18 years of age or older, and insured by either Amerigroup or UnitedHealth. These participants $(n=370)$ comprised the full analysis set (FAS) for whom data were analyzed.

Demographic characteristics of the FAS are displayed in Table 4. The mean (standard deviation $[S D]$ ) age at index of the FAS was $43.0[12.3]$ years and $45.7 \%$ were male. AmeriGroup served as the primary insurer for $81.6 \%$ while $18.4 \%$ were enrolled in UnitedHealth. At index date, 329 (88.9\%) of participants were in low intensity services such as assertive community treatment (ACT) or ACT case management style programs, which provided approximately two mental health visits per month at the CMHC. Another $23(6.2 \%)$ participants were in medium intensity community treatment team (CTT) programs, offering approximately four mental health visits per month. Finally $18(4.9 \%)$ were in high intensity services such as program of assertive community treatment 
(PACT) or PACT case management, which consisted of approximately six mental health visits per month to the CMHC.

Table 4

Baseline Demographic Characteristics

\begin{tabular}{ll}
\hline Characteristic & $\begin{array}{l}\text { FAS } \\
(n=370)\end{array}$ \\
\hline Age, mean years $(S D)$ & $43.0(12.3)$ \\
Gender, male $(\%)$ & $169(45.7 \%)$ \\
Insurance plan & \\
$\quad$ AmeriGroup, $n(\%)$ & $302(81.6 \%)$ \\
$\quad$ UnitedHealth, $n(\%)$ & $68(18.4 \%)$ \\
Level of service & $329(88.9 \%)$ \\
$\quad$ Low, $n(\%)$ & $23(6.2 \%)$ \\
$\quad$ Medium, $n(\%)$ & $18(4.9 \%)$ \\
$\quad$ High, $n(\%)$ & $40.8(47.5)$ \\
Time pre-index, mean $(S D)$ & $20.9(7.3)$ \\
Time post-index, mean $(S D)$ &
\end{tabular}

\section{Risk Adjustment}

Primary psychiatric diagnosis and Charlson Comorbidity Index (CCI) score during the pre-IHC enrollment period, as defined by International Classification of Diseases, Ninth Edition, Clinical Modification (ICD-9-CM) codes, were the main risk stratification variables. Participants were divided into three categories based on psychiatric diagnosis: (a) psychotic disorders, including schizophrenia, schizoaffective disorder, and bipolar disorder; (b) mood disorders such as depression or anxiety; and (c) substance abuse in conjunction with another psychiatric disorder (Ettner et al., 1998; Ettner et al., 2000). The majority of participants (74.3\%) in the FAS had a primary psychiatric diagnosis of psychotic disorder, while $20.8 \%$ had mood disorders and .3\% had a co-occurring substance use disorder (Table 5). Overall comorbidity was assessed 
using the CCI, a standard measure of comorbidity and identifies 17 comorbid conditions predictive of mortality and future excess costs (Charlson, Wells, Ullman, King, \& Shmukler, 2014; D’Hoore, Bouckaert, \& Tilquin, 1996). Comorbidity burden was assessed in the pre-index period six months prior to enrolling in the IHC. The mean $(S D)$ CCI score was .005 (.104), with a maximum value of 2.00 (Table 5). Of note, only one participant had preenrollment utilization that met the ICD-9-CM criteria for a CCI condition. The other participants were coded as " 0 " for CCI score and the mean CCI was calculated for the entire sample.

Table 5

Risk Stratification Variables

\begin{tabular}{ll}
\hline Variable & $\begin{array}{l}\text { FAS } \\
(n=370)\end{array}$ \\
\hline Primary psychiatric diagnosis & $275(74.3 \%)$ \\
Psychotic disorders, $n(\%)$ & $77(20.8 \%)$ \\
Mood disorders, $n(\%)$ & $1(.3 \%)$ \\
Concomitant substance use, $n(\%)$ & $14(3.8 \%)$ \\
Other, $n(\%)$ & $3(.8 \%)$ \\
Missing, $n(\%)$ & $.005(.104)$ \\
CCI score, mean $(S D)$ & \\
\hline
\end{tabular}

\section{Descriptive Statistics}

Descriptive statistics were run for the utilization variables (Table 6). Overall physical health inpatient admissions, inpatient days, and ED visits were those with an ICD-9-CM code on the authorization for a physical health diagnosis. ACS-specific conditions were defined as conditions that could have been treated in an outpatient setting if appropriate access was available, and included asthma, chronic obstructive pulmonary disease, congestive heart failure, diabetes, and hypertension (Anderson \& Knickman, 
2001). Physical health pre-and post-index inpatient admissions and ED visits were low in this study sample, as were post-index ACS-specific inpatient admissions and ED visits. There was no pre-index ACS-specific utilization of any kind.

Table 6

Pre- and Post-IHC Index Utilization

\begin{tabular}{lcccc}
\hline Utilization & Min & Max & Mean $(S E)$ & $S D$ \\
\hline $\begin{array}{l}\text { Pre-index physical health inpatient } \\
\text { admissions }\end{array}$ & 0.00 & 1.00 & $0.008(.047)$ & 0.090 \\
$\begin{array}{l}\text { Post-index physical health inpatient } \\
\text { admissions }\end{array}$ & 0.00 & 5.00 & $0.051(.018)$ & 0.353 \\
$\begin{array}{l}\text { Pre-index physical health inpatient days } \\
\text { Post-index physical health inpatient days }\end{array}$ & 0.00 & 2.00 & $0.011(.007)$ & 0.127 \\
$\begin{array}{l}\text { Pre-index physical health ED visits } \\
\text { Post-index physical health ED visits }\end{array}$ & 0.00 & 51.00 & $0.297(.150)$ & 2.877 \\
$\begin{array}{l}\text { Pre-index ACS-specific inpatient } \\
\text { admissions }\end{array}$ & 0.00 & 17.00 & $0.073(.019)$ & 0.372 \\
Post-index ACS-specific inpatient & $0.003(.076)$ & 0.00 & $0.000(.000)$ & 0.000 \\
admissions & 0.00 & 2.00 & $0.014(0.008)$ & 0.156 \\
$\begin{array}{l}\text { Pre-index ACS-specific inpatient days } \\
\text { Post- index ACS-specific inpatient days }\end{array}$ & 0.00 & 0.00 & $0.000(0.000)$ & 0.000 \\
$\begin{array}{l}\text { Pre-index ACS-specific ED visits } \\
\text { Post-index ACS-specific ED visits }\end{array}$ & 0.00 & 0.00 & $0.097(0.082)$ & 1.576 \\
& 0.00 & 2.00 & $0.000(0.000)$ & 0.000 \\
\end{tabular}

\section{Results}

\section{Research Question 1: Overall Physical Health Service Utilization}

The first research question was: What is the predictive relationship, if any, between IHC enrollment and overall physical health utilization for consumers with SMI when controlling for demographic characteristics and disease severity? The null hypothesis was that there was no statistically significant predictive relationship between IHC enrollment and overall physical health service utilization when controlling for 
demographic characteristics (age and gender) and disease severity (primary psychiatric diagnosis and CCI score). The alternative hypothesis was that there was a statistically significant predictive relationship between IHC enrollment and overall physical health service utilization when controlling for demographic characteristics and disease severity.

Paired-samples t-tests were planned; however, due to the skewed nature of the data, Wilcoxon signed-rank tests were run instead, as these are the non-parametric equivalent of paired-samples t-tests (Field, 2013). Simple linear regression, multiple linear regression, and Poisson regression analyses were also planned. However, the since the primary predictor variable in the regression was binary (pre/post enrollment in the IHC), the simple linear regression analyses were not run, as they require a continuous predictor variable (Laerd Statistics, 2015a).

Wilcoxon Signed-Rank test. Of the 370 participants, three experienced a decrease in overall physical health inpatient admissions and inpatient days post-index, whereas 11 had an increase, and 356 had no change. There were statistically significant median increases in physical health inpatient admissions $(z=2.433, p=.015)$ and inpatient days $(z=2.833, p=.005)$ following IHC enrollment compared to before IHC enrollment, as per Wilcoxon signed-rank tests. Of the 370 participants, 15 experienced a decrease in physical health ED visits post-index, whereas 109 had an increase, and 246 experienced no change. There were also statistically significant median increase in overall physical health ED visits following IHC enrollment compared to before, $z=$ 7.547, $p<.0005$ according to the Wilcoxon signed-rank test.

Logistic regression. Initially, I ran stepwise logistic regression analyses to 
examine the odds of participants experiencing any physical health inpatient admission or ED visit pre- and post-index. For the logistic regression models, counts of inpatient admissions and ED visits were recoded into a bivariate variable where " 0 " = no admission/visit and "1" = any admission/visit. Inpatient admissions and ED visits were assessed separately for pre- and post-index periods so the analysis file had two rows per participant, one for pre-index and one for post-index, with either a 0 or 1 indicating whether or not the participant experienced a physical health inpatient admission or ED visit during each one of those periods. IHC enrollment was examined first as the sole fixed effect with age, gender, initial psychiatric diagnosis, and CCI score added to the fixed-effects portion of the models. Separate analyses were run for inpatient admissions and ED visits.

Physical health inpatient admissions. There were 13 studentized residuals with values outside of two standard deviations above or below the mean. These were retained in the analyses following examination of the data and a determination that the outliers were flagged for having positive values for inpatient admissions. Since admissions were coded as " 0 " for no admission and " 1 " for any admission, transforming the data was not possible. Stepwise logistic regression was run entering IHC enrollment as the sole first effect, followed by the other variables in the second step. The model using IHC enrollment alone was the best fit as a predictor of overall inpatient admissions $\left(X^{2}(1,4.948), p=.026\right)$. The model indicated that between $3.9 \%$ and $7.7 \%$ of the variance in the criterion was explained by IHC enrollment depending on the use of the Cox \& Snell $R^{2}(.077)$ or the Nagelkerke $R^{2}(.039)$. The model correctly predicted the odds of 
any physical health inpatient admissions $98.1 \%$ of the time, while it successfully predicted $100 \%$ of the "no inpatient admission" category, and $0 \%$ of the "any inpatient admission" category. The second model adding age, gender, initial psychiatric diagnosis, and CCI score to the original model did not improve the ability to predict physical health admissions $\left(X^{2}(8,12.361), p=.136\right)$. The odds ratios for the first block are reported in Table 7.

Table 7

Odds Ratio for Experiencing Any Physical Health Inpatient Admission

\begin{tabular}{lccccc} 
& & & & \multicolumn{2}{c}{$95 \%$ CI for $\operatorname{Exp}(B)$} \\
& $B$ & $p$-value & $\operatorname{Exp}(B)$ & Lower & Upper \\
\hline IHC enrollment & 1.321 & .044 & 3.748 & 1.037 & 13.547 \\
Constant & -4.807 & .000 & 0.008 & & \\
\hline
\end{tabular}

Although the model statistically significantly predicted any physical health admission $(p=.026)$, the percentage of overall variance explained by the model was low and none of the "any admissions" category could be explained; therefore, the null hypothesis was retained.

Physical health ED visits. I ran a stepwise logistic regression model to predict the odds of a participant experiencing any physical health ED visit. There were 17 studentized residuals with values outside of two standard deviations above or below the mean. As in the first model, these cases were retained in the analysis. Although the first block of the logistic regression was a good fit to describe IHC enrollment as a predictor of a physical health ED visit $\left(X^{2}(1,94.657), p<.001\right)$, adding age, gender, initial psychiatric diagnosis and CCI score to the original model improved its statistical 
significance $\left(X^{2}(8,115.011), p<.001\right)$. This model explained between $14.4 \%$ and $24.0 \%$ of the variance in physical health ED visits. The model had an overall success of $82.7 \%$ at predicting the odds of an ED visit (.1\% less than the original predictor of enrollment). The model successfully predicted $99.3 \%$ of the "no ED visit" category, and $2.4 \%$ of the “any ED visit" category. IHC enrollment (Wald $\left.X^{2}=66.317, p<.001\right)$ and gender (Wald $X^{2}=5.717, p=.017$ ) were statistically significant predictors; however, the other variables were not. The odds ratios for the second block are reported in Table 8 . The odds ratio for IHC enrollment $(\operatorname{Exp} B=10.150, \mathrm{CI}[5.811,17.729])$ indicated that the odds of a physical health ED visit increased tenfold post-index. The odds ratio for gender $(\operatorname{Exp} B=1.714, \mathrm{CI}[1.102,2.665])$ indicated that odds of an ED visit increased by 1.71 with female gender.

Table 8

Odds Ratios for Experiencing Any Physical Health ED Visit

\begin{tabular}{lccccc} 
& & & & \multicolumn{2}{c}{$95 \%$ CI for $\operatorname{Exp}(B)$} \\
& $B$ & $p$-value & $\operatorname{Exp}(B)$ & Lower & Upper \\
\hline IHC enrollment & 2.317 & .000 & 10.150 & 5.811 & 17.729 \\
Age & -0.017 & .064 & 0.984 & 0.966 & 1.001 \\
Gender & 0.539 & .017 & 1.714 & 1.102 & 2.665 \\
Psychosis & 0.402 & .114 & 1.495 & 0.908 & 2.461 \\
Mood disorder & 1.559 & .353 & 4.756 & 0.177 & 127.562 \\
Substance use & 0.537 & .291 & 1.711 & 0.631 & 4.639 \\
CCI score & 2.595 & .121 & 13.400 & 0.505 & 355.671 \\
Constant & -2.909 & .000 & 0.055 & & \\
\hline
\end{tabular}

Although the model itself was a good predictor of a participant experiencing any physical health ED visit, not all inputs into the model individually statistically 
significantly predicted the odds of experiencing an ED visit; therefore, the null hypothesis was retained.

Multiple linear regression. Next, I examined the predictive relationship between physical health utilization and IHC enrollment, demographic characteristics, and disease severity using multiple linear regression analyses. In the forced entry method, predictors were entered into the model simultaneously, making no decisions regarding the order of the predictors (Field, 2013). Separate regression models were run for each type of service, including inpatient admissions, inpatient days, and ED visits, resulting in three regression models for overall physical health utilization.

Initially I ran multiple regression models on the data set using original count data for the utilization variables. Although the Durbin-Watson statistics indicated that there was independence of residuals for the models, scatter plots showed evidence of nonlinearity and casewise diagnostics flagged all inpatient and ED utilization as outliers, since there were so few positive events. Therefore, a square root transformation was applied to the data to correct for moderately positively skewed data (Laerd Statistics, $2015 b)$.

Physical health inpatient admissions. In the first regression model I assessed the predictive relationship between overall physical health inpatient admissions, as measured by the square root of inpatient admissions, and IHC enrollment, demographic characteristics, and disease severity. Partial regression plots and a plot of studentized residuals against the predicted values indicated linearity, and the Durbin-Watson statistic of 2.059 indicated independence of residuals. There was homoscedasticity, as assessed by 
visual inspection of a plot of studentized residuals versus unstandardized predicted values. There was no evidence of multicollinearity, as assessed by tolerance values greater than 0.1 . There were no studentized deleted residuals greater than \pm 3 standard deviations from the mean, no leverage values greater than 0.2 , or values for Cook's distance above 1 . The normality of errors assumption was violated, as assessed by the skewed nature of the data and the $\mathrm{P}-\mathrm{P}$ plot.

The model was a good fit, $F(5,734)=2.264 ; p=.047$; however, the $R^{2}$ value indicated a small effect size, as the model accounted for $1.5 \%$ of the variance in inpatient admissions, with an adjusted $R^{2}$ value of $.8 \%$. As shown in Table 9, enrollment in the IHC $(p=.019)$ predicted inpatient admissions at a statistically significant level but none of the other variables were predictive. There was a significant positive correlation between IHC enrollment and inpatient admissions as noted by the standardized coefficient, indicating that following enrollment, inpatient admissions increased.

Table 9

Individual Predictors of Physical Health Inpatient Admissions

\begin{tabular}{lccccc}
\hline \multicolumn{1}{c}{ Model } & \multicolumn{2}{c}{ Unstandardized } & \multicolumn{2}{c}{$\begin{array}{c}\text { Standardized } \\
\text { coefficients }\end{array}$} & \multicolumn{2}{c}{ coefficients } & & \\
& $B$ & $S E$ & $B$ & $t$ & $p$-value \\
\hline (Constant) & -.002 & .033 & & -0.055 & .956 \\
IHC enrollment & .029 & .013 & .086 & 2.351 & .019 \\
Age (years) & .001 & .001 & .045 & 1.218 & .226 \\
Gender & -.018 & .013 & -.052 & -1.408 & .163 \\
Psychiatric diagnosis & .007 & .004 & .059 & 1.607 & .108 \\
CCI score & -.017 & .060 & -.010 & -0.281 & .778 \\
\hline
\end{tabular}

Although the $\mathrm{p}$-value $(p=.047)$ indicated that the model including IHC enrollment, age, gender, psychiatric diagnosis and CCI score statistically significantly 
predicted physical health inpatient admissions, the $R^{2}$ value indicated that the model only predicted $1.8 \%$ of variance. Furthermore, only one of five variables was an independent statistically significant predictor $(p=.019)$, and the assumption of normality was violated. Therefore no conclusive decision could be made about the null hypothesis.

Physical health inpatient days. The model assessing the predictive relationship between overall physical health inpatient days and IHC enrollment, demographic characteristics, and disease severity also displayed linearity, independence of residuals (as assessed by a Durbin-Watson statistic of 2.067), and homoscedasticity. There was no evidence of multicollinearity. There were four studentized deleted residuals greater than \pm 3 standard deviations, but these cases were retained in the analysis because there were no leverage values greater than 0.2 or values for Cook's distance above one. However, the normality of errors assumption was violated, as assessed by visual inspection of the histogram and the $\mathrm{P}-\mathrm{P}$ plot, even after the dependent variable had been transformed.

The model accounted for $2.3 \%$ of the variance in physical health inpatient days as indicated by the $\mathrm{R}^{2}$ value and was a good fit $(F(5,734)=3.481 ; p=.004)$; however, the model did not generalize well, as indicated by the adjusted $R^{2}$ value of .017 . As shown in Table 10, both IHC enrollment and initial psychiatric diagnosis predicted physical health inpatient days at statistically significant levels, while the other variables did not. The sign of the standardized coefficients indicated that the correlations for both variables were positive; therefore, inpatient days increased in the post-index period following IHC enrollment. As the value for initial psychiatric diagnosis increased, so did overall inpatient days. 
Table 10

Individual Predictors of Physical Health Inpatient Days

\begin{tabular}{lccccc}
\hline \multicolumn{1}{c}{ Model } & \multicolumn{2}{c}{ Unstandardized } & \multicolumn{2}{c}{$\begin{array}{c}\text { Standardized } \\
\text { coefficients }\end{array}$} & \multicolumn{2}{c}{ coefficients } & & \\
& $B$ & $S E$ & $B$ & $t$ & $p$-value \\
\hline (Constant) & -.061 & .076 & & -0.805 & .421 \\
IHC enrollment & .072 & .028 & .093 & 2.539 & .011 \\
Age (years) & .002 & .001 & .059 & 1.590 & .112 \\
Gender & -.033 & .029 & -.043 & -1.160 & .246 \\
Psychiatric diagnosis & .027 & .009 & .104 & 2.829 & .005 \\
CCI score & -.032 & .137 & -.009 & -0.233 & .816 \\
\hline
\end{tabular}

The model predicted physical health inpatient days at a statistically significant level $(p=.004)$; however, the model predicted less than $5 \%$ of variance, as indicated by the $R^{2}$ value. The assumption of normality was violated, and IHC enrollment ( $\left.p=.011\right)$ and initial psychiatric diagnosis $(p=.05)$ were the only statistically significant independent predictors of inpatient days. Therefore no conclusive decision was made about the null hypothesis.

Physical health ED visits. I used a third regression model to assess the predictive relationship between physical health ED visits, as measured by the square root transformation, and IHC enrollment, demographic characteristics, and disease severity. There was linearity, independence of residuals as assessed by a Durbin-Watson statistic of 1.771, and homoscedasticity. There was no evidence of multicollinearity. There was one studentized deleted residual greater than \pm 3 standard deviations, but this case was retained due to low utilization no leverage values greater than 0.2 or values for Cook's distance above one. As in the other two models, the normality of errors assumption was violated, as assessed by the skewed histogram and the $\mathrm{P}-\mathrm{P}$ plot. 
The model accounted for $12.1 \%$ of the variance in ED visits as assessed by the $\mathrm{R}^{2}$ value, and the model was a good fit, $F(5,734)=20.244 ; p<.001$, adjusted $R^{2}=11.5 \%$ ). As shown in Table 11, IHC enrollment, gender, and initial psychiatric diagnosis predicted ED visits at statistically significant levels, but age and CCI score did not. The sign of the standardized coefficients indicated that there was a statistically significant positive correlation between all three significant variables and ED visits. Physical health ED visits increased following IHC enrollment, with female gender, and with larger values for psychiatric disorders.

Table 11

Individual Predictors of Physical Health ED Visits

\begin{tabular}{lccccc}
\hline \multicolumn{1}{c}{ Model } & \multicolumn{2}{c}{ Unstandardized } & \multicolumn{2}{c}{ Standardized } \\
& \multicolumn{2}{c}{ coefficients } & coefficients & & \\
& $B$ & $S E$ & $B$ & $t$ & $p$-value \\
\hline (Constant) & -.054 & .099 & & -0.549 & .583 \\
IHC enrollment & .342 & .037 & .319 & 9.231 & .000 \\
Age (years) & -.002 & .002 & -.042 & -1.187 & .236 \\
Gender & .091 & .037 & .085 & 2.436 & .015 \\
Psychiatric diagnosis & .030 & .012 & .084 & 2.405 & .016 \\
CCI score & .178 & .179 & .034 & 0.993 & .321 \\
\hline
\end{tabular}

The model statistically significantly predicted physical health ED visits ( $p<$ $0.001)$ and three of the five variables were independent predictors at statistically significant levels (IHC enrollment, $p<.001$; gender, $p=.015$; initial psychiatric diagnosis, $p=.016)$. However, according to the $R^{2}$ value, only $12 \%$ of the variance in the model was explained, and visual inspection of the histogram revealed positively skewed data despite transformation of the dependent variable. Therefore no conclusive decision could be made about the null hypothesis. 
Poisson regression. Poisson regression analyses were also used to examine the predictive relationship between IHC enrollment and overall physical health service utilization because the dependent variables were count data. IHC enrollment was examined first as the sole fixed effect, with age, gender, initial psychiatric diagnosis, and CCI score added to the models. Separate Poisson regression models were run for overall physical health inpatient admissions, inpatient days, and ED visits. However, when initially run, the CCI score caused Hessian matrix singularity due to the low number of participants with a CCI score. Therefore, this variable was removed from all models and the models were re-fit to include only age, gender, and initial psychiatric diagnosis in the second step of the model (Laerd Statistics, 2015c).

The Poisson regression to predict physical health inpatient admissions that included IHC enrollment, age, gender, and initial psychiatric diagnosis showed slight overdispersion (Pearson chi-square $=2.034)$ but was statistically significant, per the omnibus test. IHC enrollment, age, and initial psychiatric diagnosis statistically significantly predicted inpatient admissions. In the post-index period, $0.158(95 \% \mathrm{CI}$, 0.047 to 0.534$)$ times more inpatient admissions occurred $(p=.003)$. For every additional year of age, $1.038(95 \%$ CI, 1.002 to 1.075$)$ times more inpatient admissions occurred ( $p$ $=.040)$. For every additional increase in initial psychiatric diagnosis, 1.331 (95\% CI, 1.158 to 1.530$)$ times more inpatient admissions occurred $(p<.001)$. Since not all of the independent variables statistically significantly predicted inpatient admissions, the null hypothesis was retained. 
For the Poisson regression to predict the number of overall physical health inpatient days based on enrollment in the IHC, the model including IHC enrollment, age, gender, and initial psychiatric diagnosis was the best predictor. Overdispersion was noted but to a lesser degree than in the model with IHC as the sole predictor variable (Pearson chi-square $=9.733)$. Following IHC enrollment, $0.036(95 \% \mathrm{CI}, 0.013$ to 0.099$)$ times more inpatient days occurred $(p<.001)$. For every additional year of age, $1.065(95 \% \mathrm{CI}$, 1.047 to 1.083$)$ times more inpatient days occurred $(p<.001)$. If gender was male, 0.482 ( $95 \%$ CI, 0.320 to 0.727$)$ times more inpatient days occurred $(p=.001)$. For every additional increase in initial psychiatric diagnosis, 1.569 (95\% CI, 1.490 to 1.652$)$ times more inpatient days occurred $(p<.001)$. Since the model was statistically significant, per the omnibus test, and all variables significantly predicted inpatient days, the null hypothesis was rejected.

In the Poisson regression to predict the number of overall physical health ED visits, the model including IHC enrollment, age, gender, and initial psychiatric diagnosis was the best fit to predict ED visits. Slight overdispersion was noted (Pearson chi-square $=2.695)$. The model was statistically significant, per the omnibus test, with IHC enrollment, gender, and initial psychiatric diagnosis statistically significantly predicting physical health ED visits. Following IHC enrollment, 0.121 (95\% CI, 0.081 to 0.181 ) times more ED visits occurred $(p<.001)$. If gender was female, $1.531(95 \% \mathrm{CI}, 1.179$ to $1.989)$ times more inpatient admissions occurred $(p=.001)$. For every additional increase in initial psychiatric diagnosis, 1.123 (95\% CI, 1.048 to 1.192$)$ times more inpatient admissions occurred $(p<.001)$. Since not all of the independent variables statistically 
significantly predicted inpatient admissions, the null hypothesis was retained. The table below (Table 12) presents a summary of the analyses for research question one, the statistical tests run, and the findings with relation to the null hypothesis.

Table 12

Summary of Regression Analyses for RQ1

\begin{tabular}{|c|c|c|c|}
\hline $\begin{array}{l}\text { Analysis type and } \\
\text { purpose }\end{array}$ & Variables & Result & $\begin{array}{l}\text { Null hypothesis } \\
\text { action }\end{array}$ \\
\hline \multicolumn{4}{|c|}{ Stepwise logistic regression } \\
\hline $\begin{array}{l}\text { Model 1: } \\
\text { Examined the odds of } \\
\text { experiencing any } \\
\text { physical health } \\
\text { inpatient admission } \\
\text { before and after IHC } \\
\text { enrollment }\end{array}$ & $\begin{array}{l}\text { Dependent: Any } \\
\text { physical health } \\
\text { inpatient } \\
\text { admission } \\
\text { Independent: IHC } \\
\text { enrollment alone }\end{array}$ & $\begin{array}{l}\text { IHC enrollment predicted } \\
\text { inpatient admissions ( } p= \\
.026) \\
\text { Predicted less than } 5 \% \text { of } \\
\text { the model's variance }\end{array}$ & Retained \\
\hline $\begin{array}{l}\text { Model 2: } \\
\text { Examined the odds of } \\
\text { experiencing any } \\
\text { physical health ED } \\
\text { visit before and after } \\
\text { IHC enrollment }\end{array}$ & $\begin{array}{l}\text { Independent: IHC } \\
\text { enrollment, age, } \\
\text { gender, initial } \\
\text { psychiatric } \\
\text { diagnosis, CCI } \\
\text { score }\end{array}$ & $\begin{array}{l}\text { Model was good fit for } \\
\text { physical health ED visits } \\
(p<.001) \\
\text { Predicted } 14.4 \%-24.0 \% \\
\text { of the variance } \\
\text { IHC enrollment ( } p< \\
.001) \& \text { gender }(\mathrm{p}=.011) \\
\text { were only two predictors } \\
\text { of ED visit }\end{array}$ & Retained \\
\hline
\end{tabular}

Multiple linear regression using forced entry method

\begin{tabular}{|c|c|c|c|}
\hline $\begin{array}{l}\text { Model 1: } \\
\text { Examined predictive } \\
\text { relationship between } \\
\text { physical health } \\
\text { inpatient admissions } \\
\text { and IHC enrollment, } \\
\text { demographic } \\
\text { characteristics, and } \\
\text { disease severity }\end{array}$ & $\begin{array}{l}\text { Independent: IHC } \\
\text { enrollment, age, } \\
\text { gender, initial } \\
\text { psychiatric } \\
\text { diagnosis, CCI }\end{array}$ & $\begin{array}{l}\text { Model was a good fit ( } p \\
=.047) \\
\text { Adjusted } R^{2}=.08 \\
\text { IHC enrollment ( } p= \\
.019 \text { ) only statistically } \\
\text { significant predictor } \\
\text { Assumption of }\end{array}$ & $\begin{array}{l}\text { No conclusive } \\
\text { decision }\end{array}$ \\
\hline
\end{tabular}




\begin{tabular}{|c|c|c|c|}
\hline & score & \multicolumn{2}{|l|}{ normality was violated } \\
\hline $\begin{array}{l}\text { Model 2: } \\
\text { Examined predictive } \\
\text { relationship between } \\
\text { physical health } \\
\text { inpatient days and } \\
\text { IHC enrollment, } \\
\text { demographic } \\
\text { characteristics, and } \\
\text { disease severity }\end{array}$ & $\begin{array}{l}\text { Dependent: } \\
\text { Physical health ED } \\
\text { visits } \\
\text { Independent: IHC } \\
\text { enrollment, age, } \\
\text { gender, initial } \\
\text { psychiatric } \\
\text { diagnosis, CCI } \\
\text { score }\end{array}$ & $\begin{array}{l}\text { Model was a good fit ( } p \\
=.004) \\
\text { Adjusted } R^{2}=.017 \\
\text { IHC enrollment ( } p= \\
.011) \text { and initial } \\
\text { psychiatric diagnosis ( } p \\
=.05 \text { ) were statistically } \\
\text { significant predictors } \\
\text { Assumption of } \\
\text { normality was violated } \\
\text { Model was a good fit ( } p \\
<.001) \\
\text { Adjusted } R^{2}=.115 \\
\text { IHC enrollment ( } p< \\
.001) \text {, gender ( } p= \\
.015) \text { and initial } \\
\text { psychiatric diagnosis ( } p \\
=.016) \text { predicted ED } \\
\text { visits at statistically } \\
\text { significant levels } \\
\text { Assumption of } \\
\text { normality was violated }\end{array}$ & $\begin{array}{l}\text { No conclusive } \\
\text { decision }\end{array}$ \\
\hline Poisson regression & & & \\
\hline $\begin{array}{l}\text { Model 1: } \\
\text { Examined predictive } \\
\text { relationship between } \\
\text { physical health } \\
\text { inpatient admissions } \\
\text { and IHC enrollment, } \\
\text { demographic } \\
\text { characteristics, and } \\
\text { disease severity }\end{array}$ & $\begin{array}{l}\text { Dependent: } \\
\text { Physical health } \\
\text { inpatient } \\
\text { admissions } \\
\text { Independent: IHC } \\
\text { enrollment, age, } \\
\text { gender, initial } \\
\text { psychiatric } \\
\text { diagnosis }\end{array}$ & $\begin{array}{l}\text { IHC enrollment }(p= \\
.003), \text { age }(p=.040), \\
\text { and initial psychiatric } \\
\text { diagnosis }(p<.001) \\
\text { were statistically } \\
\text { significant predictors }\end{array}$ & Retained \\
\hline Model 2: & Dependent: & IHC enrollment $(p<$ & Rejected \\
\hline
\end{tabular}




\begin{tabular}{|c|c|c|c|}
\hline $\begin{array}{l}\text { Examined predictive } \\
\text { relationship between } \\
\text { physical health } \\
\text { inpatient days and } \\
\text { IHC enrollment, } \\
\text { demographic } \\
\text { characteristics, and } \\
\text { disease severity }\end{array}$ & $\begin{array}{l}\text { Physical health } \\
\text { inpatient days } \\
\text { Independent: IHC } \\
\text { enrollment, age, } \\
\text { gender, initial } \\
\text { psychiatric } \\
\text { diagnosis }\end{array}$ & $\begin{array}{l}.001), \text { age }(p=.001), \\
\text { gender }(p=.001) \text { and } \\
\text { initial psychiatric } \\
\text { diagnosis }(p<.001) \\
\text { statistically } \\
\text { significantly predicted } \\
\text { inpatient admissions }\end{array}$ & \\
\hline $\begin{array}{l}\text { Model 3: } \\
\text { Examined predictive } \\
\text { relationship between } \\
\text { physical health ED } \\
\text { visits and IHC } \\
\text { enrollment, } \\
\text { demographic } \\
\text { characteristics, and } \\
\text { disease severity }\end{array}$ & $\begin{array}{l}\text { Dependent: Any } \\
\text { physical health ED } \\
\text { visit } \\
\text { Independent: IHC } \\
\text { enrollment, age, } \\
\text { gender, initial } \\
\text { psychiatric } \\
\text { diagnosis }\end{array}$ & $\begin{array}{l}\text { IHC enrollment ( } p< \\
.001), \text { gender }(p= \\
.001) \text {, and initial } \\
\text { psychiatric diagnosis ( } p \\
<.001) \text { statistically } \\
\text { significantly predicted } \\
\text { ED visits }\end{array}$ & Retained \\
\hline
\end{tabular}

\section{Research Question 2: ACS-Specific Service Utilization}

The second research question was: What is the predictive relationship, if any, between IHC enrollment and ACS-specific utilization for consumers with SMI when controlling for demographic characteristics and disease severity? The null hypothesis was that there was no statistically significant predictive relationship between IHC enrollment and ACS-specific service utilization when controlling for demographic characteristics (age and gender) and disease severity (primary psychiatric diagnosis and CCI score). The alternative hypothesis was that there was a statistically significant predictive relationship between IHC enrollment and ACS-specific service utilization when controlling for demographic characteristics and disease severity.

As with the first research question, I ran Wilcoxon signed-rank tests in place of paired samples t-tests due to the skewed nature of the data. Simple linear regression, 
multiple linear regression, and Poisson regression analyses were planned. Since the primary predictor variable in the regression was binary (pre/post admission into the IHC), simple linear regression analyses were not run, as they require a continuous predictor variable. Furthermore, since there were only two ACS-specific inpatient admissions and two ACS-specific ED visits, all analyses were viewed with caution.

Wilcoxon Signed-Rank tests. When assessing ACS-specific inpatient admissions and days, of the 370 participants, none experienced a decrease in the post-index period following IHC enrollment, whereas three had an increase, and 367 had no change. There were no statistically significant median differences in ACS-specific inpatient admissions $(z=1.633, p=.102)$ or days $(z=1.604, p=.109)$ in the post-index period compared to the pre-index period. However, there was a statistically significant median difference in ACS-specific ED visits. Of the 370 participants, none experienced a decrease in ACSspecific ED visits following IHC enrollment, six had an increase, and 364 experienced no change. There was a statistically significant median increase in ACS-specific ED visits post-index following IHC enrollment compared to before IHC enrollment, $z=2.333, p=$ .020 .

Logistic regression. I examined the odds of participants experiencing any ACSspecific inpatient admission or ED visit pre- and post-IHC enrollment using stepwise logistic regression. Counts of ACS-specific inpatient admissions and ED visits were recoded into a bivariate variable where " $0 "=$ no ACS-specific admission/visit and " $1 "=$ any ACS-specific admission/visit. Inpatient admissions and ED visits were assessed separately for pre- and post-index timeframes so the analysis file had two rows per 
participant, one for preenrollment and one for postenrollment, with either a 0 or 1 indicating whether or not the participant experienced an ACS-specific event during each of those periods. IHC enrollment was examined first as the sole fixed effect with age, gender, initial psychiatric diagnosis, and CCI score added to the fixed-effects portion of the model. Separate analyses were run for ACS-specific inpatient admissions and ED visits.

ACS-specific inpatient admissions. There were two studentized residuals with values outside of two standard deviations above or below the mean, but these were retained following examination of the data and a determination that the outliers were flagged due to positive ACS-specific admissions. Since admissions were coded as "0" for no admission and " 1 " for any admission, transforming the data was not possible. The first regression model for IHC enrollment alone was a good fit to describe IHC enrollment as a predictor of experiencing any ACS-specific inpatient admission, $X^{2}[1,4.171], p=.041$. Adding age, gender, initial psychiatric diagnosis and CCI score improved the original model and the model was statistically significant $\left(X^{2}[8,15.815], p=.045\right)$, explaining between $1.7 \%$ and $9.7 \%$ of the variance in the criterion. The model had an overall success of $99.7 \%$ at predicting the odds of a participant experiencing any ACS-specific inpatient admission (.1\% more than the original predictor of IHC enrollment). The model successfully predicted $100 \%$ of the "no ACS-specific admission" category, and $33.3 \%$ of the "any ACS-specific admission" category. However, none of the predictor variables alone were statistically significant predictors of the odds of experiencing an ACS-specific inpatient admission (Table 13). 
Table 13

Odds Ratios for Experiencing an ACS-Specific Inpatient Admission

\begin{tabular}{lccccc} 
& & & & \multicolumn{2}{c}{$95 \%$ CI for $\operatorname{Exp}(\mathrm{B})$} \\
& $B$ & $p$-value & $\operatorname{Exp}(B)$ & Lower & Upper \\
\hline IHC enrollment & 16.335 & .993 & 12435361.9 & 0.000 & 0.004 \\
Age & -0.043 & .474 & 0.958 & 0.850 & 1.078 \\
Gender $_{\text {Psychosis }}^{\mathrm{a}}$ & -16.431 & .160 & 0.000 & 0.000 & - \\
Mood disorder $_{\text {Substance abuse }}$ & -14.176 & .995 & 0.000 & 0.000 & - \\
CCI score $^{\mathrm{b}}$ & -0.531 & 1.000 & 0.588 & 0.000 & 0.004 \\
Constant & -15.558 & .998 & 0.000 & 0.000 & - \\
\hline
\end{tabular}

${ }^{a}$ Data for missing initial psychiatric diagnosis are not represented in the table.

${ }^{b}$ The upper CI for enrollment and mood disorder were calculated based on Hanley's formula, as cited in Eypasch, Lefering, Kum, and Troidl, 1995.

The model itself was a good predictor of the odds of experiencing an ACSspecific inpatient admission $(p=.045)$, but none of the independent variables individually statistically significantly predicted the odds of any ACS-specific inpatient admission; therefore, the null hypothesis was retained.

ACS-specific ED visits. In the stepwise logistic regression to examine the odds of participants experiencing an ACS-specific ED visit, there were five studentized residuals with values outside of two standard deviations above or below the mean, which were retained following examination of the data and a determination that the outliers were flagged due to positive ED visits. IHC enrollment alone was the best predictor of ACSspecific ED visits $\left(X^{2}(1,8.367), p=.004\right)$. The model indicated that between $1.1 \%$ and $12.5 \%$ of the variance was explained by IHC enrollment depending on the use of the Cox \& Snell $R^{2}(.011)$ or the Nagelkerke $R^{2}(.125)$. The model correctly predicted the odds of experiencing ACS-specific ED visits $99.2 \%$ of the time (100\% of the "no ED visit" 
category, and $0 \%$ of the "any ED visit" category). Adding age, gender, initial psychiatric diagnosis and CCI score to the original model did not improve the significance of the model. There were six ACS-specific ED visits. The odds ratio for IHC enrollment (ExpB $=26628705.5, \mathrm{CI}[0.000])$ indicated that as enrollment increased by one, the odds of ACS-specific ED visits increased. Since the confidence intervals could not be calculated, the relationship between the two variables found in the sample was not reliable. The model was a good predictor of the odds of a participant experiencing an ACS-specific ED visit $(p=.004)$, but the model predicted less than $15 \%$ of the variance and the model was not reliable; therefore, the null hypothesis was retained.

Multiple regression. I examined the predictive relationship between ACSspecific utilization and IHC enrollment, demographic characteristics, and disease severity using multiple linear regression analyses. In the forced entry method, predictors were entered into the model simultaneously, making no decisions regarding the order of the predictors (Field, 2013). Separate regression models were run for each type of service, including inpatient admissions, inpatient days, and ED visits, resulting in three regression models for ACS-specific utilization. As with the overall physical health utilization models, scatter plots showed evidence of non-linearity and casewise diagnostics flagged all ACS-specific inpatient and ED utilization as outliers, since there were so few positive events. Therefore, I applied a square root transformation to the data to correct for moderately positively skewed data and re-ran the multiple linear regression models using the transformed variables (Laerd Statistics, 2015b).

ACS-specific inpatient admissions. In the model predicting the relationship 
between ACS-specific inpatient admissions and IHC enrollment, demographic characteristics, and disease severity, there was independence of residuals as assessed by a Durbin-Watson statistic of 2.046, and homoscedasticity. There was no evidence of multicollinearity since none of the correlations were above 0.70 and tolerance statistics were greater than 0.10 (Laerd Statistics, 2015b). There were also no studentized deleted residuals greater than \pm 3 standard deviations. However, the assumptions of normality and linearity were not met, although the dependent variable had previously been transformed. The model accounted for $2.0 \%$ of the variance as assessed by the $R^{2}$ value and was a good fit $(F(5,734)=2.961 ; p=.012)$; however, the model did not generalize well, as indicated by the adjusted $R^{2}$ value of .013 . Both gender and initial psychiatric diagnosis significantly predicted ACS-specific inpatient admissions, while the other variables did not (Table 14). The sign of the standardized coefficients for gender indicated a significant negative correlation while a positive correlation between initial psychiatric diagnosis and inpatient admissions was found.

Table 14

Individual Predictors of ACS-specific Inpatient Admissions

\begin{tabular}{lccccc}
\hline \multicolumn{1}{c}{ Model } & \multicolumn{2}{c}{ Unstandardized } & \multicolumn{2}{c}{ Standardized } \\
& Coefficients & Coefficients & & \\
& $B$ & $S E$ & $B$ & $t$ & $p$-value \\
\hline (Constant) & 0.006 & .016 & & 0.362 & .718 \\
IHC enrollment & 0.010 & .006 & 0.063 & 1.726 & .085 \\
Age (years) & $9.262 \mathrm{E}-5$ & .000 & 0.014 & 0.373 & .710 \\
Gender & -0.012 & .006 & -0.073 & -1.988 & .047 \\
Psychiatric diagnosis & 0.006 & .002 & 0.106 & 2.872 & .004 \\
CCI score & -0.005 & .029 & -0.006 & -0.162 & .871 \\
\hline
\end{tabular}


Although the p-value indicated that the model statistically significantly predicted ACS-specific inpatient admissions $(p=.012)$, the assumptions of linearity and normality were violated and the $R^{2}$ value indicated that the model predicted only $1.8 \%$ of variance so no conclusive decision could be made about the null hypothesis.

ACS-specific inpatient days. There was evidence of homoscedasticity and independence of residuals as assessed by a Durbin-Watson statistic of 2.076 in the model assessing the predictive relationship between ACS-specific inpatient days and IHC enrollment, demographic characteristics, and disease severity. There was no evidence of multicollinearity since none of the correlations were above 0.70 and tolerance statistics were greater than 0.10 (Laerd Statistics, 2015b). However, the assumptions of normality and linearity were violated, even though the dependent variable had already been transformed. There was also one studentized deleted residual greater than three standard deviations from the mean. The case was removed due to the large standard deviation statistic, and as a result, the model no longer retained independence of residuals. Therefore, the results reported are from the original model that retained the outlier case. The model accounted for $3.4 \%$ of the variance in ACS-specific inpatient days as assessed via the $R^{2}$ value and was a good fit $(F(5,734)=5.155 ; p<.0005)$ but did not generalize well, as indicated by the adjusted $R^{2}$ value of .027 . Only initial psychiatric diagnosis $(p<$ .001) statistically significantly predicted ACS-specific inpatient days (Table 15), and the sign of the standardized coefficient indicated a significant positive correlation. 
Table 15

Individual Predictors of ACS-specific Inpatient Days

\begin{tabular}{lccccc}
\hline \multicolumn{1}{c}{ Model } & \multicolumn{2}{c}{ Unstandardized } & \multicolumn{2}{c}{$\begin{array}{c}\text { Standardized } \\
\text { coefficients }\end{array}$} & \multicolumn{2}{c}{ coefficients } & & \\
& $B$ & $S E$ & $B$ & $t$ & $p$-value \\
\hline (Constant) & -0.023 & .043 & & -0.531 & .596 \\
IHC enrollment & 0.024 & .016 & 0.055 & 1.504 & .133 \\
Age (years) & 0.001 & .001 & 0.038 & 1.030 & .303 \\
Gender & -0.029 & .016 & -0.064 & -1.763 & .078 \\
Psych diagnosis & 0.024 & .005 & 0.166 & 4.539 & .000 \\
CCI score & -0.010 & .077 & -0.005 & -0.129 & .898 \\
\hline
\end{tabular}

Since the assumptions of linearity and normality were violated and the model did not generalize well as assessed by the $R^{2}$ value, I determined that the model was not a good fit and no conclusive decision was made about the null hypothesis.

ACS-specific ED visits. In the model assessing the predictive relationship between ACS-specific ED visits and IHC enrollment, demographic characteristics, and disease severity there was evidence of homoscedasticity but there was independence of residuals as assessed by a Durbin-Watson statistic of 2.030 . There was no evidence of multicollinearity, as correlations were all below 0.70 and VIF statistics were below 10 (Laerd Statistics, 2015b). There were no studentized deleted residuals greater than three standard deviations from the mean. However, the assumptions of normality and linearity were violated, despite transformation of the dependent variable. The model accounted for $1.2 \%$ of the variance in ACS-specific ED visits as assessed by the $\mathrm{R}^{2}$ value and the model was not a good fit $(F(5,734)=1.851 ; p=.101)$. Only enrollment in the IHC significantly predicted ACS-specific ED visits (Table 16), and the sign of the standardized coefficient indicated a significant positive correlation, meaning that ACS-specific ED visits 
increased following enrollment.

Table 16

Individual Predictors of ACS-specific ED Visits

\begin{tabular}{lccccc}
\hline \multicolumn{1}{c}{ Model } & \multicolumn{2}{c}{ Unstandardized } & \multicolumn{2}{c}{$\begin{array}{c}\text { Standardized } \\
\text { coefficients }\end{array}$} & \\
& $B$ & $S E$ & $B$ & $t$ & $p$-value \\
\hline coefficients & -0.024 & .019 & & -1.264 & .206 \\
IHC enrollment & 0.017 & .007 & 0.055 & 2.439 & .015 \\
Age (years) & 0.000 & .000 & 0.038 & 0.828 & .408 \\
Gender & 0.005 & .007 & -0.064 & 0.708 & .479 \\
Psychiatric diagnosis & 0.004 & .002 & 0.166 & 1.548 & .122 \\
CCI score & -0.003 & .034 & -0.005 & -0.086 & .932 \\
\hline
\end{tabular}

The model did not predict ACS-specific ED visits $(p=.101)$, and therefore the null hypothesis was retained.

Poisson regression. There was no preenrollment ACS- specific utilization, and very little postenrollment ACS- specific utilization (two inpatient admissions and two ED visits); therefore, the Poisson regression analyses could not be run without violating the Hessian matrix and no model statistics were produced (Laerd Statistics, 2015c). The table below presents a summary of the analyses for research question two, the statistical tests run, and the findings with relation to the null hypothesis.

Table 17

Summary of Regression Analyses for RQ2

\begin{tabular}{llll}
\hline \multicolumn{1}{c}{$\begin{array}{c}\text { Analysis type and } \\
\text { purpose }\end{array}$} & \multicolumn{1}{c}{ Variables } & \multicolumn{1}{c}{ Result } & $\begin{array}{c}\text { Null } \\
\text { hypothesis } \\
\text { action }\end{array}$ \\
\hline Logistic regression & & & \\
\hline $\begin{array}{l}\text { Model 1: } \\
\begin{array}{l}\text { Examined odds of } \\
\text { experiencing any }\end{array}\end{array}$ & $\begin{array}{l}\text { Dependent: Any } \\
\text { ACS-specific }\end{array}$ & $\begin{array}{l}\text { Full model was best fit to } \\
\text { inpatient admission }\end{array}$ & $\begin{array}{l}\text { Retained } \\
\text { admission }(p=.045)\end{array}$ \\
\end{tabular}




ACS-specific
inpatient admission
before and after IHC
enrollment

Model 2:
Examined odds of
experiencing any
ACS-specific ED
visit before and after
IHC enrollment

Independent: IHC

enrollment, age, gender, initial

psychiatric

diagnosis, CCI score

Dependent: Any

ACS-specific ED

visit

Independent: IHC

enrollment alone
Explained $9.7 \%$ of the

variance in the criterion

No variables were

independent predictors

IHC enrollment alone Retained

was a good fit to predict

odds of ACS-specific ED

visit $(p=.026)$

$1.1 \%-12.5 \%$ of the

model's variance

explained

Multiple linear regression using forced entry method

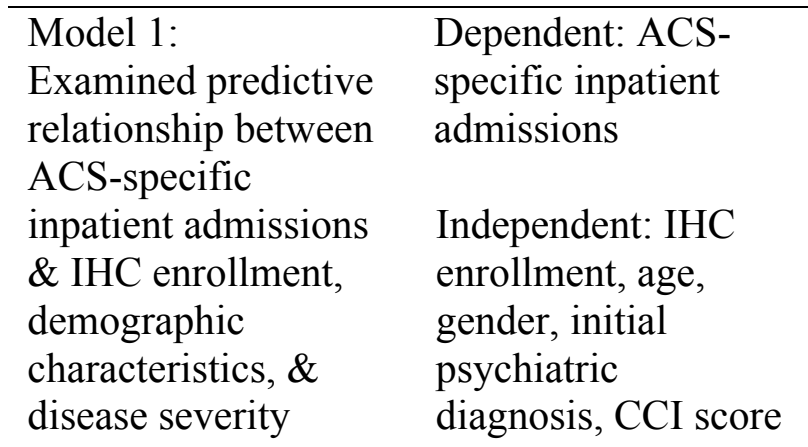

Model was a good fit $(p$ $=.012$ )

No conclusive

Adjusted $R^{2}=.013$

Gender $(p=.047) \&$

initial psychiatric

diagnosis $(p=.004)$

were statistically

significant predictors

Assumptions of linearity

$\&$ normality violated

Model 2:

Examined predictive

relationship between

ACS-specific

inpatient days \& IHC

enrollment,

demographic

characteristics, \&

disease severity
Dependent: ACSspecific inpatient days

Independent: IHC enrollment, age, gender, initial psychiatric diagnosis, CCI score
Model was a good fit ( $p$ $<.0005$ )

No conclusive decision
Adjusted $R^{2}=.027$

Initial psychiatric diagnosis $(p<.001)$ was a statistically significant predictor

Assumptions of linearity \& normality violated; Homoscedasticity \& 
independence of residuals

Model 3:

Examined predictive relationship between ACS-specific ED visits \& IHC enrollment, demographic characteristics, \& disease severity
Dependent: ACSspecific ED visits

Independent: IHC enrollment, age, gender, initial psychiatric diagnosis, CCI score
Model was not a good fit $(p=.101)$

Adjusted $R^{2}=.012$

IHC enrollment ( $p=$ .015) was only statistically significant predictor

Assumptions of linearity \& normality violated; Homoscedasticity, independence of residuals

\begin{tabular}{|c|c|c|c|}
\hline Poisson regression & & & \\
\hline $\begin{array}{l}\text { Model 1: } \\
\text { Examined predictive } \\
\text { relationship between } \\
\text { ACS-specific inpatient } \\
\text { admissions and IHC } \\
\text { enrollment, } \\
\text { demographic } \\
\text { characteristics, and } \\
\text { disease severity }\end{array}$ & $\begin{array}{l}\text { Dependent: ACS- } \\
\text { specific inpatient } \\
\text { admissions } \\
\text { Independent: IHC } \\
\text { enrollment, age, } \\
\text { gender, initial } \\
\text { psychiatric diagnosis }\end{array}$ & $\begin{array}{l}\text { Hessian matrix } \\
\text { violated due to no } \\
\text { preenrollment } \\
\text { ACS utilization; } \\
\text { analyses not run }\end{array}$ & Not applicable \\
\hline $\begin{array}{l}\text { Model 2: } \\
\text { Examined predictive } \\
\text { relationship between } \\
\text { ACS-specific inpatient } \\
\text { days and IHC } \\
\text { enrollment, } \\
\text { demographic } \\
\text { characteristics, and } \\
\text { disease severity }\end{array}$ & $\begin{array}{l}\text { Dependent: ACS- } \\
\text { specific inpatient days } \\
\text { Independent: IHC } \\
\text { enrollment, age, } \\
\text { gender, initial } \\
\text { psychiatric diagnosis }\end{array}$ & $\begin{array}{l}\text { Hessian matrix } \\
\text { violated due to no } \\
\text { preenrollment } \\
\text { ACS utilization; } \\
\text { analyses not run }\end{array}$ & Not applicable \\
\hline $\begin{array}{l}\text { Model 3: } \\
\text { Examined predictive } \\
\text { relationship between } \\
\text { ACS-specific ED visits }\end{array}$ & $\begin{array}{l}\text { Dependent: ACS- } \\
\text { specific ED visits } \\
\text { Independent: IHC }\end{array}$ & $\begin{array}{l}\text { Hessian matrix } \\
\text { violated due to no } \\
\text { preenrollment } \\
\text { ACS utilization; }\end{array}$ & Not applicable \\
\hline
\end{tabular}


and IHC enrollment, demographic characteristics, and disease severity enrollment, age, gender, initial psychiatric diagnosis analyses not run

\section{Additional Analyses}

Due to the small amount of utilization within the sample, I also assessed all-cause and mental health-related utilization, using methods identical to the analyses above. As with the previous variables, there was little pre-index utilization of any kind, with the exception of mental health inpatient days. Pre-index mental health days were greater than post-index mental health days. For all other service categories, post-index utilization exceeded pre-index utilization (Table 18).

Table 18

Pre- and Post-Index Utilization Descriptive Statistics: Additional Variables

\begin{tabular}{lcccc}
\hline Utilization & Min & Max & Mean $(S E)$ & $S D$ \\
\hline Pre-index all-cause inpatient admissions & 0.00 & 2.00 & $0.016(.009)$ & 0.164 \\
Post-index all-cause inpatient admissions & 0.00 & 9.00 & $0.143(.038)$ & 0.731 \\
Pre-index all-cause inpatient days & 0.00 & 48.00 & $0.168(.133)$ & 2.561 \\
Post-index all-cause inpatient days & 0.00 & 63.00 & $0.995(.279)$ & 5.360 \\
Pre-index all-cause ED visits & 0.00 & 4.00 & $0.087(.022)$ & 0.427 \\
Post-index all-cause ED visits & 0.00 & 18.00 & $0.754(.088)$ & 1.687 \\
Pre-index mental health inpatient & 0.00 & 2.00 & $0.081(.006)$ & 0.116 \\
admissions & & & & \\
Post-index mental health inpatient & 0.00 & 9.00 & $0.092(.030)$ & 0.582 \\
admissions & & & & \\
Pre-index mental health inpatient days & 0.00 & 48.00 & $0.157(.132)$ & 2.548 \\
Post-index mental health inpatient days & 0.00 & 43.00 & $0.697(.214)$ & 4.108 \\
Pre-index mental health ED visits & 0.00 & 2.00 & $0.014(.008)$ & 0.156 \\
Post-index mental health ED visits & 0.00 & 6.00 & $0.105(.026)$ & 0.496 \\
\hline
\end{tabular}

All-cause utilization. Of the 370 participants, four experienced a decrease in inpatient admissions and inpatient days post-index, whereas 18 had an increase, and 350 
had no change. There were statistically significant median increases in all-cause inpatient admissions $(z=3.597, p>.0005)$ and inpatient days $(z=3.665, p>.0005)$ as indicated by Wilcoxon signed-rank tests. There was also a statistically significant median increase in $\mathrm{ED}$ visits $(z=8.605, p>.0005)$ following IHC enrollment.

Logistic regression. In the logistic regression analysis assessing the odds of a participant experiencing any all-cause inpatient admission, there were 27 studentized residuals with values outside of two standard deviations of the mean, which were retained for analysis. The full model including all covariates was a statistically significant improvement over the original model using IHC enrollment alone, $X^{2}(8,29.645), p<$ .001 , explaining between $3.9 \%$ and $13.6 \%$ of the variance in the criterion. The improved model had an overall success of $95.9 \%$ at predicting the odds of experiencing an all-cause inpatient admission (100\% of the "no admission" category and $0 \%$ of the "any admission" category). IHC enrollment (Wald $X^{2}=12.922, p<.001$ ) remained a statistically significant predictor of the odds of an all-cause inpatient admission, but none of the new variables were individually statistically significant predictors. Since only IHC enrollment individually predicted the odds of experiencing an all-cause inpatient admission at statistically significant level, the null hypothesis was retained.

There were 19 studentized residuals with values outside of two standard deviations of the mean in the logistic regression analysis assessing the odds of a participant experiencing any all-cause ED visit; however, these cases were retained following a determination that the outliers were flagged due to positive utilization. The model that included IHC enrollment, age, gender, initial psychiatric diagnosis, and CCI 
score best predicted a consumer experiencing an all-cause ED visit $\left(X^{2}(8,128.273), p<\right.$ .001 ), explaining between $15.9 \%$ and $25.5 \%$ of the variance in the criterion. The model had an overall success of $80.0 \%$ at predicting the odds of any all-cause ED visit $(97.8 \%$ of the "no ED visit" category and $4.9 \%$ of the "any ED visit" category). IHC enrollment (Wald $\left.X^{2}=76.027, p<.001\right)$, age (Wald $\left.X^{2}=7.232, p=.007\right)$ and gender (Wald $X^{2}=$ $5.364, p=.021)$ were statistically significant predictors. However, since not all variables individually statistically significantly predicted the odds of experiencing any $\mathrm{m}$ ED visit, the null hypothesis was retained.

Multiple linear regression. I assessed the predictive relationship between allcause utilization, and IHC enrollment, demographic characteristics, and disease severity using multiple linear regression. The models for inpatient admissions $(p<.001)$ and ED visits $(p=.001)$ were good fits. IHC enrollment $(p<.001)$ and age $(p=.032)$ were statistically significant predictors of inpatient admissions with age being a negative predictor. All variables except initial psychiatric diagnosis were predictive of all-cause ED visits; however, they were not representative as indicated by adjusted $R^{2}$ values accounting for low levels of variance. As such, I determined that the models were not good fits and no conclusive decision could be made about the null hypotheses. The model for all-cause inpatient days was not a good fit $(p=.201)$ and none of the variables were independent predictors; therefore the null hypothesis was retained. Furthermore, the assumptions of linearity and normality were violated in all three models.

Poisson regression. Poisson regression models were used to predict the number of all-cause inpatient admissions, inpatient days, and ED visits based on IHC enrollment 
as the sole first effect, and adding age, gender, and initial psychiatric diagnosis to the models. In the first model, IHC enrollment and initial psychiatric diagnosis predicted an increase in all-cause inpatient admissions. Following IHC enrollment, 0.113 (95\% CI, 0.049 to 0.263$)$ times more all-cause inpatient admissions occurred $(p<.001)$ and for every additional increase in initial psychiatric diagnosis, 1.140 (95\% CI, 1.014 to 1.280$)$ times more admissions occurred $(p=.028)$. In the second model, IHC enrollment, age, and initial psychiatric diagnosis significantly predicted all-cause inpatient days.

Following IHC enrollment, 0.168 (95\% CI, 0.129 to 0.220$)$ times more inpatient admissions occurred $(p<.001)$, with each additional year of age, 0.978 (95\% CI, 0.971 to 0.986) times more all-cause inpatient days occurred $(p<.001)$, and for each additional increase in initial psychiatric diagnosis, 1.177 (95\% CI, 1.133 to 1.224$)$ times more allcause inpatient days occurred $(p<.001)$; however the model was subject to overdispersion (Pearson chi-square $=26.901)$. In the final Poisson regression model IHC enrollment, gender, and initial psychiatric diagnosis predicted an increase in all-cause ED visits. Following IHC enrollment, 0.115 (95\% CI, 0.080 to 0.165$)$ times more all-cause ED visits occurred $(p<.001)$. When gender was male, 1.487 (95\% CI, 1.177 to 1.879$)$ times more ED visits occurred $(p=0.001)$. For each additional increase in initial psychiatric diagnosis, 1.144 (95\% CI, 1.055 to 1.177$)$ times more ED visits occurred ( $p<$ .001). The null hypotheses were retained for all Poisson regression analyses since not all predictor variables statistically significantly predicted all-cause utilization (Table 21). 
Table 19

Summary of Regression Analyses for All-Cause Utilization

\begin{tabular}{|c|c|c|c|}
\hline $\begin{array}{c}\text { Analysis type and } \\
\text { purpose }\end{array}$ & Variables & Result & $\begin{array}{c}\text { Null hypothesis } \\
\text { action }\end{array}$ \\
\hline \multicolumn{4}{|c|}{ Stepwise logistic regression } \\
\hline $\begin{array}{l}\text { Model 1: } \\
\text { Examined the odds } \\
\text { of experiencing any } \\
\text { inpatient admission } \\
\text { before and after } \\
\text { IHC enrollment }\end{array}$ & $\begin{array}{l}\text { Dependent: Any } \\
\text { inpatient admission } \\
\text { Independent: IHC } \\
\text { enrollment, age, } \\
\text { gender, initial } \\
\text { psychiatric diagnosis, } \\
\text { CCI score }\end{array}$ & $\begin{array}{l}\text { Model was a good fit ( } p \\
<.001) \\
3.9 \%-13.6 \% \text { of the } \\
\text { variance in the criterion } \\
\text { explained } \\
\text { IHC enrollment ( } p< \\
.001) \text { was a statistically } \\
\text { significant predictor of } \\
\text { odds of an all-cause } \\
\text { inpatient admission }\end{array}$ & Retained \\
\hline $\begin{array}{l}\text { Model 2: } \\
\text { Examined the odds } \\
\text { of experiencing any } \\
\text { ED visit before and } \\
\text { after IHC } \\
\text { enrollment }\end{array}$ & $\begin{array}{l}\text { Dependent: Any ED } \\
\text { visit } \\
\text { Independent: IHC } \\
\text { enrollment, age, } \\
\text { gender, initial } \\
\text { psychiatric diagnosis, } \\
\text { CCI score }\end{array}$ & $\begin{array}{l}\text { Model was a good fit ( } p \\
<.001) \\
\text { Explained } 15.9 \% \text { - } \\
25.5 \% \text { of the variance in } \\
\text { the criterion } \\
\text { IHC enrollment }(p< \\
.001), \text { age }(p=.007) \& \\
\text { gender }(p=.021) \text { were } \\
\text { statistically significant } \\
\text { predictors }\end{array}$ & Retained \\
\hline \multicolumn{4}{|c|}{ Multiple linear regression } \\
\hline $\begin{array}{l}\text { Model 1: } \\
\text { Examined predictive } \\
\text { relationship between } \\
\text { all-cause inpatient } \\
\text { admissions \& IHC } \\
\text { enrollment, } \\
\text { demographic } \\
\text { characteristics, \& } \\
\text { disease severity }\end{array}$ & $\begin{array}{l}\text { Independent: IHC } \\
\text { enrollment, age, } \\
\text { gender, initial } \\
\text { psychiatric } \\
\text { diagnosis, CCI } \\
\text { score }\end{array}$ & $\begin{array}{l}\text { Model was a good fit ( } p \\
=.001) \\
\text { Adjusted } R^{2}=.022 \\
\text { IHC enrollment }(p< \\
.001) \& \text { age }(p=.032) \\
\text { were statistically } \\
\text { significant predictors } \\
\text { (age }=\text { negative } \\
\text { predictor) }\end{array}$ & $\begin{array}{l}\text { No conclusive } \\
\text { decision } \\
\text { reached }\end{array}$ \\
\hline
\end{tabular}




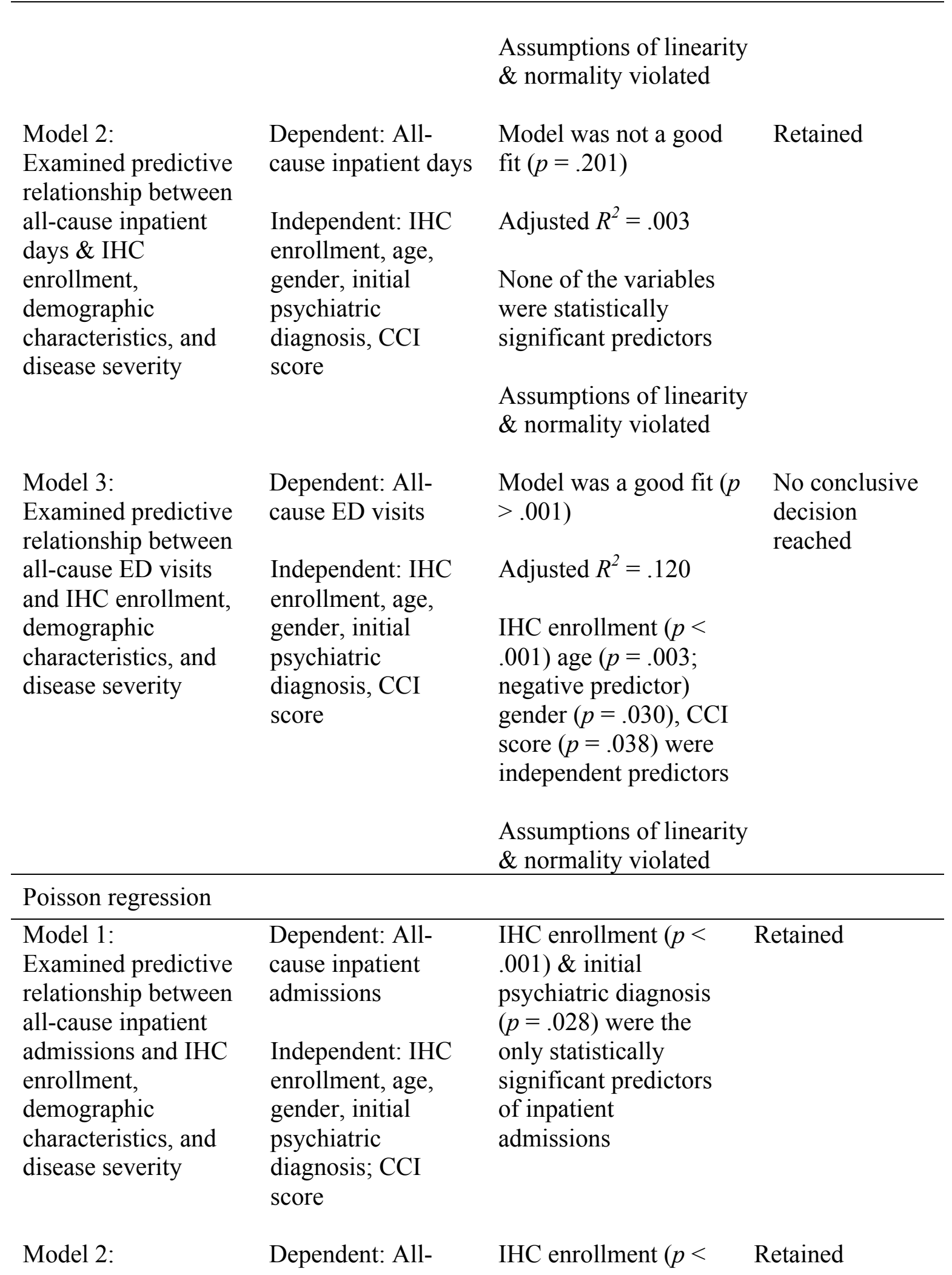


Examined predictive relationship between all-cause inpatient days \& IHC enrollment, demographic characteristics, \& disease severity

Model 3:

Examined predictive relationship between all-cause ED visits \& IHC enrollment, demographic characteristics, \& disease severity cause inpatient days .001$)$, age $(p<.001)$, $\&$ initial psychiatric Independent: IHC diagnosis $(p<.001)$ enrollment, age, were statistically gender, initial psychiatric diagnosis; CCI score significant predictors of inpatient days

Overdispersion was noted

Dependent: All- $\quad$ IHC enrollment $(p<\quad$ Retained cause ED visits $\quad .001)$, age $(p=.001)$, $\&$ initial psychiatric Independent: IHC diagnosis $(p<.001)$ enrollment, age, were statistically gender, initial significant predictors psychiatric of ED visits diagnosis; CCI score

Mental health utilization. When analyzing mental health utilization, two of 370 participants experienced a decrease in overall mental health inpatient admissions and inpatient days following enrollment, whereas 18 had an increase, and 350 had no change. There were statistically significant median increases in mental health inpatient admissions $(z=3.189, p=.01)$ and mental health ED visits $(z=3.381, p=.01)$ following IHC enrollment compared to before IHC enrollment as assessed by Wilcoxon signedrank tests. However, there was a statistically significant decrease in inpatient days $(z=$ 2.784, $p=.005$ ) following IHC admission compared to before IHC admission.

Logistic regression. In the stepwise logistic regression analysis assessing the odds of experiencing any mental health inpatient admission, there were 17 studentized residuals with values outside of two standard deviations of the mean; however these were retained for analysis following examination of the data and a determination that the 
outliers were flagged due to positive utilization. The model including all covariates was the best predictor of experiencing a mental health inpatient admission $\left(X^{2}(8,29.990), p<\right.$ $.001)$. Between $4.0 \%$ and $18.1 \%$ of the variance in the criterion could be explained and the model had an overall success of $97.3 \%$ at predicting any mental health admission, predicting $100 \%$ of the "no mental health admission" category and $0 \%$ of the "any mental health admission" category. IHC enrollment (Wald $X^{2}=9.870, p=.002$ ) and age (Wald $\left.X^{2}=8.011, p=.005\right)$ were statistically significant predictors, but none of the other variables were; therefore the null hypothesis was retained.

The logistic regression for mental health ED visits included 25 studentized residuals with values outside of two standard deviations of the mean, and these cases were retained following a determination that the outliers were flagged due to positive ED visits. The model including IHC enrollment, age, gender, initial psychiatric diagnosis, and CCI score improved the significance of the original model $\left(X^{2}(8,26.534), p=.001\right)$ and was retained as the best predictor, explaining between $3.5 \%$ and $13.4 \%$ of the variance in the criterion. The model had an overall success of $96.5 \%$ at predicting the odds of participants experiencing mental health ED visits while successfully predicting $100.0 \%$ of the "no mental health ED visit" category and $0 \%$ of the "any mental health ED visit" category. IHC enrollment (Wald $X^{2}=8.304, p=.001$ ) remained a statistically significant predictor of the odds of experiencing a mental health ED visit, but none of the other variables were statistically significant predictors; therefore, the null hypothesis was retained. 
Multiple linear regression. I assessed the predictive relationship between mental health utilization, and IHC enrollment, demographic characteristics, and disease severity through multiple linear regression. The models for mental health inpatient admissions $(p$ $<.001)$, inpatient days $(p=.006)$, and ED visits $(p=.001)$ significantly fit the data, but were not representative, as less than $5.0 \%$ of the variance was accounted for according to the $R^{2}$ values. IHC enrollment was a statistically significant predictor of all three utilization variables while age was a statistically significant negative predictor of mental health inpatient admissions and days. The assumptions of linearity and normality were also violated in all three models; therefore it was determined that the models were not good fits and no conclusive decisions could be made about the null hypotheses.

Poisson regression. In the Poisson regression assessing mental health inpatient admissions, the model that included IHC enrollment, age, gender, and initial psychiatric diagnosis was statistically significant but IHC enrollment and age were the only variables to significantly predict mental health admissions. Following IHC enrollment, 0.088 (95\% CI, 0.027 to 0.287$)$ times fewer mental health inpatient admissions occurred $(p<.001)$. For every additional year increase in age, 0.965 (95\% CI, 0.938 to 0.992$)$ times more admissions occurred $(p=.012)$. The second model assessing mental health inpatient days was subject to overdispersion (Pearson chi-square $=22.244$ ). IHC enrollment, age and initial psychiatric diagnosis significantly negatively predicted an increase in mental health inpatient days. Following IHC enrollment, 0.225 (95\% CI, 0.169 to 0.299$)$ times fewer mental health inpatient days were incurred $(p<.001)$, for every additional decrease in age, $0.939(95 \% \mathrm{CI}, 0.930$ to 0.949$)$ times fewer mental health days occurred $(p<$ 
$.001)$, and for every additional decrease in initial psychiatric diagnosis, 0.885 (95\% CI, 0.814 to 0.962$)$ times fewer mental health inpatient days occurred $(p=.004)$. In the Poisson regression model assessing mental health ED visits, IHC enrollment predicted an increase in ED visits. Following IHC enrollment, 0.128 (95\% CI, 0.051 to 0.325$)$ times more mental health ED visits occurred $(p<.001)$. After adding age, gender, and initial psychiatric diagnosis to the model, the model remained significant but no other variables significantly predicted mental health ED visits. The null hypotheses were retained for all three models due to the fact that not all variables were statistically significant predictors of mental health utilization (Table 20).

Table 20

Summary of Regression Analyses for Mental Health Utilization

\begin{tabular}{|c|c|c|c|}
\hline $\begin{array}{c}\text { Analysis Type and } \\
\text { Purpose }\end{array}$ & Variables & Result & $\begin{array}{c}\text { Null Hypothesis } \\
\text { Action }\end{array}$ \\
\hline \multicolumn{4}{|c|}{ Stepwise logistic regression } \\
\hline $\begin{array}{l}\text { Model 1: } \\
\text { Examined odds of } \\
\text { experiencing any } \\
\text { mental health } \\
\text { inpatient admission } \\
\text { before and after IHC } \\
\text { enrollment }\end{array}$ & $\begin{array}{l}\text { Independent: IHC } \\
\text { enrollment, age, } \\
\text { gender, initial } \\
\text { psychiatric } \\
\text { diagnosis, CCI } \\
\text { score }\end{array}$ & $\begin{array}{l}\text { Model was a good fit ( } p \\
<.001) \\
4.0 \%-18.1 \% \text { of the } \\
\text { variance in criterion } \\
\text { explained } \\
\text { IHC enrollment ( } p= \\
.002) \text { and age }(p=.005) \\
\text { were statistically } \\
\text { significant predictors }\end{array}$ & Retained \\
\hline $\begin{array}{l}\text { Model 2: } \\
\text { Examined odds of } \\
\text { experiencing a mental } \\
\text { health ED visit before } \\
\text { and after IHC } \\
\text { enrollment }\end{array}$ & $\begin{array}{l}\text { Dependent: Any } \\
\text { mental health ED } \\
\text { visit } \\
\text { Independent: IHC } \\
\text { enrollment, age, } \\
\text { gender, initial }\end{array}$ & $\begin{array}{l}\text { Model was a good fit ( } p \\
=.001) \\
\text { Explained } 3.5 \%-13.4 \% \\
\text { of variance in criterion } \\
\text { IHC enrollment ( } p=\end{array}$ & Retained \\
\hline
\end{tabular}


psychiatric

diagnosis, $\mathrm{CCI}$

score

Multiple linear regression

Model 1:
Examined predictive
relationship between
mental health
inpatient admissions
\& IHC enrollment,
demographic
characteristics, \&
disease severity

disease severity
.001) was a statistically significant predictor
Model 2:

Examined predictive relationship between mental health inpatient days \& IHC enrollment, demographic characteristics, \& disease severity
Model 3:

Examined predictive relationship between mental health ED visits \& IHC enrollment, demographic characteristics, \& disease severity
Dependent: Mental Model was a good fit ( $p$ health inpatient

admissions

Independent: IHC enrollment, age, gender, initial psychiatric diagnosis, CCI score

Dependent: All- $\quad$ Model was a good fit ( $p$ cause inpatient days $=.006$ )

Independent: IHC

enrollment, age, gender, initial psychiatric diagnosis, CCI score $<.001)$

Adjusted $R^{2}=.024$

IHC enrollment $(p<$ $.001) \&$ age $(p=.003)$

were significant predictors (age was negative)

Assumptions of linearity \& normality violated

Adjusted $R^{2}=.015$

IHC enrollment ( $p=$ $.003) \&$ age $(p=.016)$

were significant predictors (age was
No conclusive decision negative)

Assumptions of linearity \& normality violated

Dependent: Mental Model was a good fit ( $p$ health ED visits =.001)

No conclusive decision

Independent: IHC Adjusted $R^{2}=.021$ enrollment, age, gender, initial psychiatric diagnosis, CCI score
No conclusive decision
IHC enrollment ( $p<$
$.001)$ was a statistically
significant predictor

Assumptions of linearity \& normality violated 


\begin{tabular}{|c|c|c|c|}
\hline \multicolumn{4}{|l|}{ Poisson regression } \\
\hline $\begin{array}{l}\text { Model 1: } \\
\text { Examined predictive } \\
\text { relationship between } \\
\text { mental health } \\
\text { inpatient admissions } \\
\text { and IHC enrollment, } \\
\text { demographic } \\
\text { characteristics, and } \\
\text { disease severity }\end{array}$ & $\begin{array}{l}\text { Dependent: Mental } \\
\text { health inpatient } \\
\text { admissions } \\
\text { Independent: IHC } \\
\text { enrollment, age, } \\
\text { gender, initial } \\
\text { psychiatric } \\
\text { diagnosis; CCI } \\
\text { score }\end{array}$ & $\begin{array}{l}\text { IHC enrollment }(p< \\
.001) \& \text { age }(p=.012) \\
\text { were statistically } \\
\text { significant predictors } \\
\text { of mental health } \\
\text { inpatient admissions }\end{array}$ & Retained \\
\hline $\begin{array}{l}\text { Model 2: } \\
\text { Examined predictive } \\
\text { relationship between } \\
\text { mental health } \\
\text { inpatient days \& IHC } \\
\text { enrollment, } \\
\text { demographic } \\
\text { characteristics, \& } \\
\text { disease severity }\end{array}$ & $\begin{array}{l}\text { Dependent: Mental } \\
\text { health inpatient } \\
\text { days } \\
\text { Independent: IHC } \\
\text { enrollment, age, } \\
\text { gender, initial } \\
\text { psychiatric } \\
\text { diagnosis; CCI } \\
\text { score }\end{array}$ & $\begin{array}{l}\text { IHC enrollment }(p< \\
.001), \text { age }(p<.001), \\
\& \text { initial psychiatric } \\
\text { diagnosis }(p=.004) \\
\text { were statistically } \\
\text { significant negative } \\
\text { predictors of mental } \\
\text { health inpatient days } \\
\text { Overdispersion was } \\
\text { noted }\end{array}$ & Retained \\
\hline $\begin{array}{l}\text { Model 3: } \\
\text { Examined predictive } \\
\text { relationship between } \\
\text { mental health ED } \\
\text { visits \& IHC } \\
\text { enrollment, } \\
\text { demographic } \\
\text { characteristics, \& } \\
\text { disease severity }\end{array}$ & $\begin{array}{l}\text { Dependent: Mental } \\
\text { health ED visits } \\
\text { Independent: IHC } \\
\text { enrollment, age, } \\
\text { gender, initial } \\
\text { psychiatric } \\
\text { diagnosis; CCI } \\
\text { score }\end{array}$ & $\begin{array}{l}\text { IHC enrollment ( } p< \\
.001 \text { ) was the only } \\
\text { statistically significant } \\
\text { predictor of mental } \\
\text { health ED visits }\end{array}$ & Retained \\
\hline
\end{tabular}

\section{Summary}

In summary, 370 participants met enrollment criteria of having six months of data pre- and post-index date (first visit to the IHC) available for analysis, were 18 years of age or older, and insured by either Amerigroup or UnitedHealth. For these participants, 
there was very little physical health or ACS-specific utilization, with no pre-index ACSspecific utilization of any kind. Since the data were skewed, Wilcoxon signed-rank tests were run in place of the planned paired-samples $t$-tests. Furthermore, the planned simple linear regression analyses were not run due to the fact that the independent variable was binary in nature. I ran logistic regression analyses examine the odds of participants experiencing any utilization by category before and after IHC enrollment. For these analyses, enrollment was examined first as the sole fixed effect with age, gender, initial psychiatric diagnosis, and CCI score added to the fixed-effects portion of the model. I next examined the predictive relationship between service utilization and IHC enrollment, demographic characteristics, and disease severity using multiple linear regression analyses. For the multiple regression models, a square root transformation was applied to the data to correct for moderately positively skewed data (Laerd Statistics, 2015b) as a result of evidence of non-linearity and flagging of positive utilization. I also used Poisson regression analyses to examine the predictive relationship between IHC enrollment and service utilization since the data were count data. IHC enrollment was the sole fixed effect, with age, gender, initial psychiatric diagnosis, and CCI score added to the models. However, when initially run, the CCI score caused Hessian matrix singularity due to the low number of participants with a CCI score. Therefore, this variable was removed from all models and the models were re-fit to include only age, gender, and initial psychiatric diagnosis in the second step of the model (Laerd Statistics, 2015c). 


\section{Summary: Research Question 1 - Physical Health Service Utilization}

RQ1 was: What is the predictive relationship, if any, between IHC enrollment and overall physical health utilization for consumers with SMI when controlling for demographic characteristics and disease severity? According to the Wilcoxon signedrank tests for the first research question, there were statistically significant median increases in physical health inpatient admissions, inpatient days, and ED visits following the index date compared to before. Results from the logistic regression models indicated that IHC enrollment alone was a statistically significant predictor of the odds of participants experiencing any inpatient admission and ED visit. When age, gender, psychiatric diagnosis and CCI score were added to the models, they remained good predictors; however, not all inputs into the models individually statistically significantly predicted the odds of experiencing utilization; therefore, the null hypotheses were retained in favor of the alternative hypothesis for both models.

For the multiple linear regression models assessing physical health inpatient admissions, inpatient days, and ED visits, the models were good fits but not representative due to small $R^{2}$ values. Therefore, no conclusive decisions could be made about the null hypotheses. Results of the Poisson regression models were mixed. The models for inpatient admissions and ED visits were predictive, with small increases in utilization occurring following the index date (IHC enrollment). The models remained significant after adding the covariates, with age and initial psychiatric diagnosis significantly predicting inpatient admissions, age, gender and initial psychiatric diagnosis significantly predicting inpatient days, and gender and initial psychiatric diagnosis 
significantly predicting physical health ED visits. Since not all of the covariates were statistically significantly predictive, the null hypotheses were retained. The Poisson regression model for inpatient days was statistically significant, per the omnibus test, and all variables significantly predicted inpatient days; therefore the null hypothesis was rejected in favor of the alternative hypothesis.

\section{Summary: Research Question 2 - ACS-Specific Service Utilization}

RQ2 was: What is the predictive relationship, if any, between IHC enrollment and ACS-specific utilization for consumers with SMI when controlling for demographic characteristics and disease severity? According to results of the Wilcoxon signed-rank tests for the second research question, there were no statistically significant median differences in ACS-specific inpatient admissions or days following IHC enrollment, but there was a statistically significant median difference in ACS-specific ED visits. The logistic regression models for ACS-specific inpatient admissions and ED visits were not good predictors of the odds of utilization, and none of the inputs into the model individually statistically significantly predicted the odds of ACS-specific inpatient admission or ED visits; therefore, the null hypotheses were retained.

Although the multiple linear regression models for ACS-specific inpatient admissions and days were statistically significant, the assumptions of linearity and normality violated and the models did not generalize well as evidenced by low $\mathrm{R}^{2}$ values. Therefore, no conclusive decisions were made about the null hypotheses. The multiple linear regression for ACS-specific ED visits was not statistically significant, and 
therefore the null hypothesis was retained. Due to the scarcity of ACS-specific utilization, no model statistics were produced for the Poisson regression analyses.

\section{Summary: Additional Analyses}

I ran post hoc analyses for all-cause and mental health inpatient and ED utilization, using the same methods as in the key research questions. As with the previous variables, there was low pre-index utilization for all variables with the exception of mental health inpatient days. Pre-index mental health days were greater than post-index mental health days. For all other service categories, post-index utilization exceeded preindex utilization. There were statistically significant median increases in all-cause inpatient admissions, days, and ED visits following IHC enrollment compared to before IHC enrollment.

IHC enrollment alone was a statistically significant predictor of experiencing an all-cause inpatient admission or ED visit in the logistic regression analyses. When covariates were added to the models, although the models were good predictors of the independent variables, not all inputs into the models individually statistically significantly predicted utilization; therefore, the null hypotheses was upheld in favor of the alternative hypotheses for both all-cause utilization models. Although the multiple linear regression models were good fits for the all-cause utilization data, these models accounted for less than $5.0 \%$ of variance in the all-cause utilization variables as evidenced by low $R^{2}$ values and no conclusive decisions were made about the null hypotheses. Poisson regression model results indicated that following IHC enrollment, statistically significant (although small) increases were seen in inpatient admissions, 
days, and ED visits. When covariates were added to the models, they remained significant, but not all covariates were statistically significant predictors so the null hypotheses were retained. Additionally, the model for all-cause inpatient days had a high degree of overdispersion.

There were also statistically significant median increases in mental health inpatient admissions and ED visits following IHC enrollment, as evidenced Wilcoxon signed-rank tests, and a statistically significant decrease in mental health inpatient days following IHC enrollment. IHC enrollment alone was a statistically significant predictor of experiencing both a mental health inpatient admission and an ED visit in the logistic regression models. When covariates were added to the models they remained good predictors, but not all inputs into the models individually statistically significantly predicted the odds of utilization; therefore, the null hypotheses were upheld in favor of the alternative hypotheses for both models. The multiple linear regression models for mental health utilization were good fits, but were not representative due to $R^{2}$ values of less than $5.0 \%$, and therefore no conclusive decision were made about the null hypotheses. Finally, in the Poisson regression models, that IHC enrollment predicted an increase in mental health admissions and ED visits and a decrease in inpatient days. Following IHC enrollment, small but statistically significant increases were observed in inpatient admissions and ED visits, while a small but statistically significant decrease was seen in mental health inpatient days. When covariates were added to the models, they remained significant, but again, not all of the covariates were independent predictors, and 
the model for mental health inpatient days had a high degree of overdispersion. As a result, the null hypotheses were retained for all three models.

Chapter 5 provides an interpretation of these findings, including a comparison with what has been found in peer-reviewed literature and the theoretical framework. I discuss limitations of the study in detail, as well as recommendations for future research. Finally, social change implications are explored. 
Chapter 5: Discussion, Conclusions, and Recommendations

\section{Introduction}

This quantitative retrospective cohort design study evaluated the effect of an integrated physical health clinic (IHC) within a large community mental health center (CMHC). The research questions focused on the relationship between IHC enrollment and physical health and ambulatory care sensitive (ACS)-specific service utilization for consumers with serious mental illness (SMI) when controlling for demographic characteristics and disease severity. Despite expectations, IHC enrollment did not have a significant predictive relationship with inpatient or emergency department (ED) utilization for physical health or ACS-specific conditions. As assessed in post-hoc analyses, IHC enrollment also did not have a significant predictive relationship with allcause or mental health utilization. This chapter presents a summary of the analyses, interpretation of the findings, limitations of the findings, and implications for future research.

The sample included 370 participants who met enrollment criteria; the number was adequate given the study methodology. There were few physical health or ACSspecific inpatient admissions or ED visits in the study sample, an unexpected finding. Physical health utilization was defined by the presence of an International Classification of Diseases, Ninth Edition, Clinical Modification (ICD-9-CM) code for a physical health diagnosis on the authorization. Of the 370 participants meeting study criteria (six months of data pre- and post index date [defined as the first IHC visit] and insured by AmeriGroup or UnitedHealth), 149 participants had an inpatient or ED visit for any 
reason pre- or post-IHC enrollment. Of these 149 participants, $132(40.2 \%)$ had at least one physical health inpatient or ED visit in the sampling timeframe, with higher rates of utilization following IHC enrollment. Since the data were skewed, results proved inconclusive. Results for each of the research questions are summarized below.

The first research question assessed the relationship between physical health service utilization and enrollment in the IHC, and was: What is the predictive relationship, if any, between IHC enrollment and overall physical health utilization for consumers with SMI when controlling for demographic characteristics and disease severity? There were statistically significant median increases in physical health inpatient admissions, inpatient days, and ED visits following IHC enrollment, as evidenced by Wilcoxon signed-rank tests. IHC enrollment was the best predictor of inpatient admissions and ED visits in the logistic regression models, but the models failed to remain good predictors of utilization following addition of covariates and the null hypotheses were retained for all three models. Multiple linear regression models assessing physical health inpatient admissions and days were statistically significant, but $R^{2}$ values were small and no conclusions were made about the null hypotheses. The model assessing physical health ED visits was not a good fit and the null hypothesis was retained. The Poisson regression models for physical health inpatient admissions and ED visits were predictive, with small increases in utilization occurring following IHC enrollment. The models remained significant after adding the covariates, but not all of the covariates were statistically significantly predictive, and the null hypotheses were retained. The Poisson regression model for physical health inpatient days was statistically 
significant, per the omnibus test, and all variables significantly predicted inpatient days; therefore the null hypothesis was rejected in favor of the alternative hypothesis.

The second research question assessed the impact of IHC enrollment on ACSspecific utilization, and was stated as: What is the predictive relationship, if any, between IHC enrollment and ACS-specific utilization for consumers with SMI when controlling for demographic characteristics and disease severity? ACS conditions were defined as conditions that could have been treated in an outpatient setting if appropriate access were available, and included asthma, chronic obstructive pulmonary disease, congestive heart failure, diabetes, and hypertension (Anderson \& Knickman, 2001). There were no statistically significant median differences in ACS-specific inpatient admissions or days following IHC enrollment, but there was a statistically significant median increase in ACS-specific ED visits. The logistic regression models for ACS-specific inpatient admissions and ED visits were not good predictors of the odds of utilization, and not all of the inputs into the model individually statistically significantly predicted the odds of ACS-specific inpatient admission or ED visits; therefore, the null hypotheses were retained. Although the multiple linear regression models for ACS-specific inpatient admissions and days were statistically significant, the assumptions of linearity and normality violated and the models did not generalize well as evidenced by low $R^{2}$ values. Therefore, no conclusive decisions were made about the null hypotheses. The multiple linear regression for ACS-specific ED visits was not statistically significant, and therefore the null hypothesis was retained. Poisson regression analyses were not run due to the low amount of ACS-specific utilization in the sample. 
Additional analyses mirroring the methodology used for the above two research questions were run for all-cause and mental health inpatient and ED utilization. There were statistically significant median increases in all-cause utilization following IHC enrollment. IHC enrollment alone was a statistically significant predictor of all-cause inpatient admissions and ED visits in the logistic regression models, but not all inputs into the models individually statistically significantly predicted utilization and the null hypotheses were retained. Multiple linear regression models for all-cause inpatient admissions, inpatient days, and ED visits significantly fit the data; however, the $R^{2}$ values accounted for less than $5.0 \%$ of variance in each model and no conclusive decisions were made about the null hypotheses. In the Poisson regression models, there was a statistically significant relationship between IHC enrollment and inpatient admissions, days, and ED visits. However, the model for all-cause inpatient days had a high degree of overdispersion, and not all covariates were statistically significant predictors, so the null hypotheses were retained for these models.

There were statistically significant median increases in mental health inpatient admissions and ED visits, and a statistically significant median decrease in mental health inpatient days following IHC enrollment. IHC enrollment alone was a statistically significant predictor of both mental health inpatient admissions and ED visits in the logistic regression models, but after adding other covariates, not all inputs into the models individually statistically significantly predicted utilization. As such, the null hypotheses were retained for both models. The multiple linear regression models for mental health utilization significantly fit the data, but were not representative as 
evidenced by small $R^{2}$ values and no conclusive decisions were made about the null hypotheses. IHC enrollment predicted decreases in mental health admissions and ED visits and an increase in inpatient days. The models remained significant following addition of the covariates but not all covariates were independent predictors of utilization and the null hypotheses were retained.

\section{Interpretation of Findings}

The sample in this study had much lower levels of physical health utilization than would have been expected. Prior research has shown increased inpatient and ED utilization and costs among the SMI population (Gerrity, 2015). For example, authors of a study of Medicaid claims data from Massachusetts found that comorbid physical illness was common among consumers with SMI. In that study, $74 \%$ of consumers with SMI had at least one chronic medical condition, while half were diagnosed with two or more and $50 \%$ of the sample was treated for a physical health comorbidity (Jones et al., 2004). Data for hospital admissions and readmissions in 2012 from the Agency for Healthcare Research and Quality (AHRQ) indicated that consumers with mood disorders experienced 269.8 inpatient admissions per 100,000 while consumers with schizophrenia experienced 121.9 per 100,000 population with average lengths of stay of 6.6 and 10.4 days, respectively (Heslin \& Weiss, 2015). In a commercially-insured population with schizophrenia, inpatient admissions were found to occur at a rate of 636 admissions per 1,000 population and ED visits occurred at a rate of 2,240 visits per 1,000 population. While the majority of inpatient admissions were for psychiatric conditions, $67 \%$ of the ED visits were for non-psychiatric conditions (Fitch, Iwasaki, \& Villa, 2014). Authors of 
another study using data from two EDs found that there was a mean of 2.3 ED visits per year among consumers with psychiatric conditions, and 6.4 among the population with co-occurring psychiatric conditions and alcohol abuse (Minassian, Vilke, \& Wilson, 2013). However, in this study, mean visits in both the pre- and postenrollment timeframes were less than one.

Several reasons may explain why there were fewer inpatient or ED visits among the sample in this study than expected. First, consumers may have switched health plans from their index health plan (either AmeriGroup or UnitedHealth) to a health insurer that did not provide authorization data back to the CMHC. Inpatient admissions and ED visits would not have been captured for the new health plan, as only AmeriGroup and UnitedHealth shared authorization data with the CMHC; however consumers were chosen for the study based on their index health plan. A second possibility concerns the psychiatric care given by the CMHC. The CMHC that provided data for this study was in the process of becoming a federally qualified health center (FQHC) for the SMI population that they serve, and therefore had many innovative programs underway to reduce admissions. Physical health utilization may have been impacted by one of these other programs, resulting in lower levels of utilization than what would be expected among the SMI population at large (CMHC Chief Information Officer, Personal communication, July 19, 2016). Intensive psychiatric case management, including Assertive Community Treatment (ACT) has been associated with a reduction in hospitalization regardless of physical healthcare provided (Gerrity, 2016). Third, some of the participants may have been seeking physical healthcare from other providers outside 
of the CMHC/IHC setting resulting in lowered physical health inpatient and ED care. Those visits would not have been captured in the data available for this study.

The lack of ACS-specific utilization in this study was particularly surprising. Increased ACS-specific utilization among populations of consumers with mental illness has been shown in prior research. Authors of a study of Maryland Medicaid data found that mental illness was associated with $32 \%$ higher odds of experiencing an ACS-specific hospital admission among adults aged 18-64, as well as among children and older adults (McGinty \& Sridhara, 2014). These findings confirmed the findings from two previous studies in the SMI population regarding the prevalence of ACS-specific conditions in mental illness (Cahoon, McGinty, Ford, \& Daumit, 2013; Li, Glance, Cai, \& Mukamel, 2008). I found very little ACS-specific utilization, with just two inpatient admissions and two ED visits in the sample during the one year study timeframe. Although the same ICD-9-CM codes were used to identify ACS-specific utilization as in prior research, authors of the prior studies used administrative claims data with primary and secondary diagnoses codes as the basis for analysis (Cahoon et al., 2013; Li et al., 2008; McGinty \& Sridhara, 2014). I relied on ICD-9-CM codes on authorization data provided to the CMHC from two health plans, and there was only one code (the primary diagnosis code) per authorization. It is possible that the codes were incorrect, or that an ACS-specific condition was not the primary complaint and therefore not captured in the data available to me. 


\section{IHC Impact on Utilization}

I found no statistically significant impact of IHC enrollment on inpatient or ED utilization. Findings from previous research have varied regarding the ability of integrated care to impact utilization measures. Authors of a recent randomized study assessed a physical health program within a behavioral health home CMHC, with care provided by a nurse practitioner and some level of coordination and consultation with the mental health treatment teams (Druss et al., 2016). This level of care coordination was similar to that provided within the CMHC that I studied. In that study, 447 consumers with SMI were randomized to either the health home or usual care. Similarly to results of the current study, enrollment in the behavioral health home was not associated with differences in inpatient or ED utilization but was associated with improvement in some measures related to quality of care (Druss et al., 2016). Similarly, authors of a metaanalysis assessing randomized controlled trials of integrated care programs found an increase in the use of outpatient services and improved quality of life associated with integrated care while findings were mixed in relation to inpatient service utilization and $\operatorname{cost}$ (Gerrity, 2015).

In this study, there were statistically significant median increases in both physical health and ACS-specific ED visits following IHC enrollment, but results from the modeling were mixed regarding a predictive relationship to IHC enrollment. In the logistic regression models, the null hypotheses were retained, indicating no statistically significant predictive relationship between IHC enrollment and physical health or ACSspecific ED visits. No specific conclusions could be drawn from the multiple regression 
models. These trends held true for all-cause and mental health ED visits as well. Through this study, I confirmed findings from two studies within the Veteran's Health Administration (VHA) that collocated care did not impact ED utilization. In the first study, collocated care within a behavioral health VHA clinic was associated with significantly increased outpatient visits following enrollment but no significant differences in ED service utilization were found (Pirraglia et al., 2012). Authors of a second cohort study within the VHA system assessed the association of patient-aligned care teams (PACT) and utilization of healthcare services by veterans with posttraumatic stress disorder (PTSD; Randall, Mohr, \& Maynard, 2014). In that study, PACT was associated with a reduction of inpatient admissions and specialty services but not with changes in mental health visits, ED visits, or urgent care visits (Randall et al., 2014).

\section{Theoretical Context}

I used the Chronic Care Model (CCM) as the theoretical foundation of this study. Several key aspects of the CCM informed the concepts supporting this study assessing integration of physical healthcare into behavioral health settings for consumers with SMI (Woltmann et al., 2012). Specifically, aspects of CCM related to redesigning healthcare delivery systems and improving clinical information systems aligned with the concept of integrated physical and behavioral healthcare (McLellan et al., 2014). These aspects of $\mathrm{CCM}$ also related to the concepts of Level 5 and 6 integrated care as described by Heath and colleagues (2013). Level 5 care included development and implementation of collaborative treatment planning between collocated physical and behavioral healthcare 
providers while Level 6 care referred to collaborative treatment planning for all consumers including fully integrated information systems (Heath et al., 2013).

In my study, there was no indication that outcomes were improved due to a realignment of the healthcare system within the $\mathrm{CMHC}$, although behavioral and physical healthcare were provided in a collocated manner. One explanation for these findings may be explained by the disparate data systems used for this study. Two different electronic medical record (EMR) systems were employed by the CMHC, one for the mental health clinic and one for the IHC. Furthermore, while the CMHC received authorizations for inpatient and ED utilization from two of the three health plans, the authorization data were received in Excel format each day, and did not integrate with either of the CMHC's EMR systems. Therefore, the information systems were not fully integrated and may have impacted the ability of the providers at the CMHC to adequately monitor consumers' health.

The CCM also pointed to the need for collaboration among healthcare professionals to adequately care for patients, treating the person as a whole rather than each condition in isolation (Rush, 2014). Therefore, theorists have hypothesized that improved outcomes may result from relationships between motivated patients and treatment teams who treat proactively (Barr et al., 2003). This study did not assess the motivation levels of consumers in the $\mathrm{CMHC}$, nor did it assess the specific kinds of treatment provided by either the mental health practitioners or the IHC. 


\section{Limitations of the Findings}

The primary limitation of the study was associated with the data sources used and the scarcity of utilization data among the sample. First, the study used secondary data to assess whether IHC enrollment was associated with physical health inpatient and ED service utilization. There may have been other important indicators of IHC success or confounding variables not accounted for that were not available for analysis in this study. However, use of administrative data for this study may allow other researchers to more closely replicate the methodology in assessing the success of other integrated care programs (Frankfort-Nachmias \& Nachmias, 2008). Second, I assessed inpatient and ED utilization from authorization data sent to the CMHC by two health plans. These data were provided to the $\mathrm{CMHC}$ to help providers better manage its consumers, and not for research purposes. Other administrative data may have contained better information, such as administrative claims that typically include more ICD-9-CM codes for each claim. The authorization data files were limited to one primary code only. Further, other payers covering consumers of the $\mathrm{CMHC}$ did not provide authorization data to the $\mathrm{CMHC}$ and there may have been differences in service utilization between members enrolled in the two health plans sending data compared with those that did not. Moreover, the sharing of real-time authorization data between a health plan and a CMHC may be atypical and therefore may limit the ability of other researchers to reproduce the exact methodology used in the current study. 


\section{Recommendations}

I used a pre- post design to assess change in utilization before and after enrollment in an integrated physical health program. As mentioned above, selection bias limited generalizability of the findings as there were likely differences between the consumers enrolling in the IHC and those not enrolling (Frankfort-Nachmias \& Nachmias, 2008). A comparison of consumers enrolling in integrated care programs and those not enrolling may be a more useful comparison for future research. Exploring the differences in consumers receiving care in integrated care models and those not, including the effect of those differences on service utilization may provide important insights for integrated care providers and policy makers.

Researchers should also continue to assess integrated healthcare within community behavioral health settings since the majority of consumers with SMI utilize behavioral health clinics as their primary sources of medical care (Manderscheid \& Kathol, 2014). The majority of evidence about these programs to date has come from the VHA, and findings have been mixed in terms of improvement in outcomes and resource utilization (Druss, Rohrbaugh, et al., 2001b; Kilbourne et al., 2009; Kilbourne et al., 2011). However, the VHA system serves a selected group of consumers who may not be reflective of the general United States population in terms of demographic characteristics including gender, race, or age (Goulet et al., 2007; Pirraglia et al., 2012). Authors of a meta-analysis of studies of integrated care interventions in various settings among consumers with SMI found low to medium levels of evidence supporting these interventions (McGinty et al., 2016), and this study did not produce findings that were 
reliable enough to form any solid conclusions due to low levels of utilization in the study sample. Since no two clinics are alike, research should continue on the effectiveness of integrated care programs within community behavioral health settings to determine the true extent of integrated care on service utilization, especially as recent health policy has focused on integrated care as a new treatment model (Barry \& Huskamp, 2011; Druss \& Mauer, 2010; SAMHSA, 2014).

The lack of ACS-specific data in this study made drawing conclusions about the impact of IHC enrollment on utilization difficult. Results from past research indicated that collocated care might positively impact ACS-specific utilization. Pirragalia and colleagues (2011) found that fewer consumers within the VHA system at sites with collocated medical care had hospitalizations for ACS-specific conditions compared to consumers at other VHA sites ( $4.3 \%$ vs. $5.1 \%$, respectively, $\mathrm{p}=0.004$; Pirraglia et al., 2011). Future research on the ability of integrated care programs within the community to reduce ACS-specific care would be beneficial, since the VHA system is not representative of community care.

Future research assessing the relationship between the number of visits with an integrated care provider and physical health utilization may be useful as well. I did not include an analysis of the number of postenrollment IHC visits on service utilization. However, the regular receipt of outpatient primary care may be as important for consumer health as a reduction in inpatient and ED use. Researchers of earlier studies have suggested a relationship between suboptimal access to primary care and an increase in ED use between 1997and 2007 among Medicaid beneficiaries (Tang et al., 2010). 
Increased ED use has also been associated with lack of regular general practitioner visits after controlling for demographic characteristics (McCusker et al., 2010).

In addition to assessing the impact of integrated care on inpatient and ED service use, future researchers may also wish to incorporate outpatient and preventive care as study endpoints. I did not assess these categories of service utilization in the current study, as these data were not available to me. Authors of earlier studies found link between integrated care programs and increased use of outpatient services, certain physical health screening measures, and improved quality of life but little impact on higher levels of service utilization (Druss et al., 2016; Gerrity, 2015). While highintensity service utilization such as inpatient admissions and ED visits may be more important to payer cost-reduction efforts, increased outpatient care and achievement of health goals may be of greater importance to clinicians and patients. Aligning study outcomes with different stakeholder groups' interests may add to the overall level of evidence for integrated care.

Lastly, as mentioned above, this study did not assess several important aspects of integrated care as related to the CCM, including motivation levels of consumers or intensity of treatment provided by either the mental health or physical health practitioners. Future researchers should take these aspects into consideration when assessing the impact of integrated care programs. Furthermore, it is important for consumers with SMI to have both a behavioral health provider and a physical health provider who consult with one another regarding care (Druss, Rohrbaugh, et al., 2001; Manderscheid \& Kathol, 2014). I did not assess the level of consultation or collaboration 
between the behavioral health and IHC staff regarding patient care as this information was not available in the data sets provided. In the future, researchers should assess the level of collaboration, since this is an important aspect in the success of integrated care programs as indicated by earlier research (Gerrity, 2015).

\section{Implications}

Despite the limitations of this study, findings may help inform the data partner CMHC in some important ways. There was no evidence that IHC enrollment was statistically significantly predictive of physical health service utilization. However, inpatient and ED service utilization in this study was lower than would have been expected based on prior epidemiologic studies. The fact that there was very little inpatient or ED utilization, either prior to or following IHC enrollment, may indicate that the services provided by the $\mathrm{CMHC}$ as a whole are effective in managing the health of consumers. The CMHC was applying to become a certified FQHC. This designation means that the CMHC would need to provide comprehensive services to underserved populations, and as such would qualify for enhanced funding from Medicare and Medicaid (United States Department of Health and Human Services, n.d.). Researchers have shown an association between FQHCs and fewer inpatient admissions and ED visits for ACS-specific conditions in a dual-eligible population (Wright, Potter, \& Trivedi, 2015). Leaders at the CMHC may choose to further investigate the numbers of inpatient and ED visits among their consumers in comparison to regional or national FQHCs and conduct an assessment of the total package of care provided to determine the impact on consumers. 
The lack of data available for this study may also point to an opportunity for improvement within the $\mathrm{CMHC}$ related to information management systems and the ability to adequately assess consumer outcomes. First, the IHC and mental health clinic used separate, nonintegrated EMR systems. Fully integrated Level 6 care involves fully integrated systems as well as close collaboration between physical and mental healthcare providers (Heath et al., 2013). Fully integrated systems may help reduce barriers and improve communication among providers (Grol, Wensing, Bosch, Hulscher, \& Eccles, 2013; Malm, Ivarsson, \& Allebeck, 2014; Pincus et al., 2015). Administrators within the CMHC may want to consider better alignment of care through use of a single EMR system for both mental and physical health management.

A second data issue had to do with underutilization of the IHC EMR. The IHC EMR contained fields to record results of physical health screenings such as cholesterol, hemoglobin A1c, and lipid tests; however these fields were not used and no results were recorded. Therefore, progress toward clinical goals could not be tracked over time as an outcome of this study, or by care teams at the CMHC in routine care monitoring of consumers. Assessing barriers to appropriate use of the IHC EMR may help improve future monitoring of the physical health of consumers at the $\mathrm{CMHC}$ and provide other ways to measure the impact of the IHC apart from resource utilization.

I had hoped that this study would add to the growing body of evidence supporting integrated care models. Quality of care for consumers with SMI has been a focus of recent health policies and financing structures/grant programs for integrated care pilots (Barry \& Huskamp, 2011; Druss \& Mauer, 2010; SAMHSA, 2014). Additionally, the 
Center for Medicare and Medicaid Services (CMS) and other payers are moving toward quality-based reimbursement rather than quantity-based reimbursement. Given these changes in the healthcare landscape, an understanding of the impact of integrated care programs on high-cost service utilization and quality of care to support policy and financing are necessary (Burwell, 2015). Unfortunately data issues limited the conclusiveness of the findings of this study and its ability to inform the debate on integrated care in either a positive or negative way.

\section{Conclusions}

In this study, I evaluated the effect of an integrated physical health program within a community behavioral health setting on physical health inpatient admissions and ED visits. Contrary to expectation, IHC enrollment did not have a significant predictive relationship with inpatient or ED utilization. While the sample size was adequate, there were fewer physical health or ACS-specific inpatient admissions or ED visits than would have been expected based on prior research. Since the data were skewed, results were tenuous and inconclusive. A meta-analysis of interventions for medical conditions among consumers with SMI found low to medium levels of evidence supporting these interventions, and called for further research on implementation strategies in real-world settings (McGinty et al., 2016). Although I had hoped that findings from the current study would help fill this need, it has added to the conflicting findings supporting the use of integrated care models on service utilization. Nevertheless, policy and payment structures continue to support integrated care models, and therefore further research of different programs are encouraged, as each setting and practice pattern is unique. Identifying those 
settings and programs that improve outcomes, including reductions in high-cost service utilization, increased routine care, and better health will add to the growing body of evidence to build effective models of integrated care. 
References

Agency for Healthcare Research and Quality. (2015, March). Prevention quality indicators overview. Retrieved from www.qualityindicators.ahrq.gov

Alakeson, V., Pande, N., \& Ludwig, M. (2010). A plan to reduce emergency room "boarding" of psychiatric patients. Health Affairs, 29(9), 1637-1642. doi:10.1377/hlthaff.2009.0336

Anderson, G., \& Knickman, J. R. (2001). Changing the chronic care system to meet people's needs. Health Affairs, 20(6), 146-160. doi:10.1377/hlthaff.20.6.146

Austin, B., Wagner, E., Hindmarsh, M., \& Davis, C. (2000). Elements of effective chronic care: A model for optimizing outcomes for the chronically ill. Epilepsy \& Behavior, 1(4), S15-S20. doi:10.1006/ebeh.2000.0105

Barr, V. J., Robinson, S., Marin-Link, B., Underhill, L., Dotts, A., Ravensdale, D., \& Salivaras, S. (2003). The expanded Chronic Care Model: An integration of concepts and strategies from population health promotion and the Chronic Care Model. Hospital Quarterly, 7(1), 73-82. doi:10.12927/hcq.2003.16763

Barry, C. L., \& Huskamp, H. A. (2011). Moving beyond parity - mental health and addiction care under the ACA. New England Journal of Medicine, 365(11), 973975. doi:10.1056/NEJMp1108649

Bauer, M. S., McBride, L., Williford, W. O., Glick, H., Kinosian, B., Altshuler, L., ... Lavori, P. (2015). Collaborative care for bipolar disorder: Part II. Impact on clinical outcome, function, and costs. FOCUS, 13(1), 85-93. doi:10.1176/appi.focus.130114 
Bewick, V., Cheek, L., \& Ball, J. (2005). Statistics review 14: Logistic regression. Critical Care, 9(1), 112-118. doi:10.1186/cc3045

Birnbaum, H. G., Cremieux, P. Y., Greenberg, P. E., LeLorier, J., Ostrander, J. A., \& Venditti, L. (1999). Using healthcare claims data for outcomes research and pharmacoeconomic analyses. Pharmacoeconomics, 16(1), 1-8. doi:10.2165/00019053-199916010-00001

Boaz, T. L., Becker, M. A., Andel, R., Van Dorn, R. A., Choi, J., \& Sikirica, M. (2013). Risk factors for early readmission to acute care for persons with schizophrenia taking antipsychotic medications. Psychiatric Services, 64, 1225-1229. doi:10.1176/appi.ps.003382012

Bodenheimer T, Lorig K, Holman H, \& Grumbach K. (2002). Patient self-management of chronic disease in primary care. Journal of the American Medical Association, 288(19), 2469-2475. doi:10.1001/jama.288.19.2469

Bodenheimer T, Wagner EH, \& Grumbach K. (2002). Improving primary care for patients with chronic illness: The chronic care model, part 2. Journal of the American Medical Association, 288(15), 1909-1914. doi:10.1001/jama.288.15.1909

Bradford, D. W., Cunningham, N. T., Slubicki, M. N., McDuffie, J. R., Kilbourne, A. M., Nagi, A., \& Williams, J. W. (2013). An evidence synthesis of care models to improve general medical outcomes for individuals with serious mental illness: A systematic review. Journal of Clinical Psychiatry, 74(8), e754-e764. doi:10.4088/JCP.12r07666 
Burt, J., Rick, J., Blakeman, T., Protheroe, J., Roland, M., \& Bower, P. (2014). Care plans and care planning in long-term conditions: a conceptual model. Primary Health Care Research \& Development, 15(04), 342-354. doi: $10.1017 / \mathrm{s} 1463423613000327$

Burwell, S. M. (2015). Setting value-based payment goals - HHS efforts to improve U.S. health care. New England Journal of Medicine, 372, 897-899. doi:10.1056/NEJMp1500445

Cahoon, E. K., McGinty, E. E., Ford, D. E., \& Daumit, G. L. (2013). Schizophrenia and potentially preventable hospitalizations in the United States: A retrospective cross-sectional study. BMC Psychiatry, 13, 37. doi:10.1186/1471-244X-13-37

Charlson, M., Wells, M. T., Ullman, R., King, F., \& Shmukler, C. (2014). The Charlson Comorbidity Index can be used prospectively to identify patients who will incur high future costs. PLoS ONE, 9(12), e112479. doi:10.1371/journal.pone.0112479

Cohen, J. (1992). Statistical power analysis. Current Directions in Psychological Science, 1(3), 98-101. doi:10.1111/1467-8721.ep10768783

Coleman, K., Austin, B. T., Brach, C., \& Wagner, E. H. (2009). Evidence on the Chronic Care Model in the new millennium. Health Affairs, 28(1), 75-85. doi:10.1377/hlthaff.28.1.75

Collins, C., Hewson, D. L., Munger, R., \& Wade, T. (2010). Evolving models of behavioral health integration in primary care. New York: Milbank Memorial Fund. Retrieved from www.milbank.org 
Connolly, J. G., Toomey, T. J., \& Schneeweiss, M. C. (2015). Metabolic monitoring for youths initiating use of second-generation antipsychotics, 2003-2011. Psychiatric Services, 66(6), 604-609. doi:10.1176/appi.ps.201400222

Creswell, J. W. (2009). Research design: Qualitative, quantitative, and mixed methods approaches (4th Edition). Thousand Oaks, CA: SAGE Publications, Inc.

Davis, K. (2010). A new era in American health care: Realizing the potential of reform. Washington, D.C.: The Commonwealth Fund.

De Hert, M., Correll, C. U., Bobes, J., Cetkovic-Bakmas, M., Cohen, D., Asai, I., ... Leucht, S. (2011). Physical illness in patients with severe mental disorders. I. Prevalence, impact of medications and disparities in health care. World Psychiatry, 10(1), 52-77. doi:10.1002/j.2051-5545.2011.tb00014.x

Deyo, R. A., Cherkin, D. C., \& Ciol, M. A. (1992). Adapting a clinical comorbidity index for use with ICD-9-CM administrative databases. Journal of Clinical Epidemiology, 45(6), 613-619. doi:10.1016/0895-4356(92)90133-8

D’Hoore, W., Bouckaert, A., \& Tilquin, C. (1996). Practical considerations on the use of the Charlson Comorbidity Index with administrative data bases. Journal of Clinical Epidemiology, 49(12), 1429-1433. doi:10.1016/S0895-4356(96)00271-5

Druss, B. G., Bradford, W. D., Rosenheck, R. A., Redford, M. J., \& Krumhold, H. M. (2001). Quality of medical care and excess mortality in older patients with mental disorders. Archives of General Psychiatry, 58(6), 565-572. doi:10.1001/archpsyc.58.6.565 
Druss, B. G., \& Mauer, B. J. (2010). Health care reform and care at the behavioral health - primary care interface. Psychiatric Services, 61(11), 1087-1092. doi:10.1176/appi.ps.61.11.1087

Druss, B. G., Rohrbaugh, R. M., Levinson, C. M., \& Rosenheck, R. A. (2001). Integrated medical care for patients with serious psychiatric illness: A randomized trial. Archives of General Psychiatry, 58(9), 861-868. doi:10.1001/archpsyc.58.9.861

Druss, B. G., Rosenheck, R. A., Desai, M. M., \& Perlin, J. B. (2002). Quality of preventive medical care for patients with mental disorders. Medical Care, 40(2), 129-136. doi:10.1097/00005650-200202000-00007

Druss, B. G., von Esenwein, S. A., Compton, M. T., Rask, K. J., Zhao, L., \& Parker, R. M. (2009). A randomized trial of medical care management for community mental health settings: The Primary Care Access, Referral, and Evaluation (PCARE) study. American Journal of Psychiatry, 167(2), 151-159. doi:10.1176/appi.ajp.2009.09050691

Druss, B. G., von Esenwein, S. A., Glick, G. E., Deubler, E., Lally, C., Ward, M. C., \& Rask, K. J. (2016). Randomized trial of an integrated behavioral health home: The Health Outcomes Management and Evaluation (HOME) study. American Journal of Psychiatry (ahead of print). doi:10.1176/appi.ajp.2016.16050507

Ettner, S. L., Frank, R. G., Mark, T., \& Smith, M. W. (2000). Risk adjustment of capitation payments to behavioral health care carve-outs: How well do existing methodologies account for psychiatric disability? Health Care Management Science, 3(2), 159-169. doi:10.1023/A:1019033105715 
Ettner, S. L., Frank, R. G., McGuire, T. G., Newhouse, J. P., \& Notman, E. H. (1998). Risk adjustment of mental health and substance abuse payments. Inquiry, 35(2), 223-239. Retrieved from http://www.jstor.org/stable/29772757

Faul, F., Erdfelder, E., Lang, A. G., \& Buchner, A. (2007). G*Power 3: A flexible statistical power analysis program for the social, behavioral, and biomedical sciences. Behavior Research Methods, 39, 175-191. doi:10.3758/bf03193146

Field, A. (2013). Discovering statistics using IBM SPSS Statistics (Fourth Edition). Thousand Oaks, CA: SAGE Publications, Inc.

Fitch, K., Iwasaki, K., \& Villa, K. F. (2014). Resource utilization and cost in a commercially insured population with schizophrenia. American Health and Drug Benefits, 7(1), 18-26. Retrieved from www.AHDBonline.com

Frankfort-Nachmias, C., \& Nachmias, D. (2008). Research methods in the social sciences (7th Edition). New York: Worth.

Frank, R. G., \& Epstein, A. M. (2014). Factors associated with high levels of spending for younger dually eligible beneficiaries with mental disorders. Health Affairs, 33(6), 1006-1013. doi:10.1377/hlthaff.2013.0769

Gerrity, M. (2015). Integrating primary care into behavioral health settings: What works for individuals with serious mental illness. Washington, D.C.: Milbank Memorial Fund. Retrieved from www.milbank.org

Gerrity, M. A. (2016). Evolving models of behavioral health integration: Evidence update 2010-2015 (No. 978-1-887748-81-0). New York, NY: Milbank Memorial Fund. Retrieved from www.milbank.org 
Glasgow, R. E., Tracy Orleans, C., Wagner, E. H., Curry, S. J., \& Solberg, L. I. (2001). Does the Chronic Care Model serve also as a template for improving prevention? The Milbank Quarterly, 79(4), 579-612. doi:10.1111/1468-0009.00222

Golberstein, E., \& Busch, S. H. (2013). Two steps forward, one step back? Implications of the Supreme Court's health reform ruling for individuals with mental illness. Journal of the American Medical Association - Psychiatry, 70(6), 567-568. doi:10.1001/jamapsychiatry.2013.25

Goulet, J. L., Erdos, J., Kancir, S., Levin, F. L., Wright, S. M., Daniels, S. M., ... Justice, A. C. (2007). Measuring performance directly using the Veterans Health Administration electronic medical record. Medical Care, 45(1), 73-79. doi:10.1097/01.mlr.0000244510.09001.e5

Greenhalgh, T. (2014). How to read a paper (Fifth Edition). Hoboken, NJ: John Wiley \& Sons.

Grol, R., Wensing, M., Bosch, M., Hulscher, M., \& Eccles, M. (2013). Theories on implementation of change in healthcare. In Improving patient care: The implementation of change in healthcare (Second Edition). Hoboken, NJ: WileyBlackwell.

Heath, B., Wise Romero, P., \& Reynolds, K. (2013). A standard framework for levels of integrated healthcare. Washington, D.C.: SAMHSA-HRSA Center for Integrated Health Solutions. Retrieved from http://www.integration.samhsa.gov/resource/ standard-framework-for-levels-of-integrated-healthcare 
Heslin, K. C., \& Weiss, A. J. (2015). Hospital readmissions involving psychiatric disorders, 2012 (Statistical Brief No. Statistical Brief \#189). Rockville, MD: Agency for Healthcare Research and Quality.

Iversen, G. R. (2004). Quantitative research. In M. Lewis-Beck, A. Bryman, \& T. Liao, Encyclopedia of social science research methods. Thousand Oaks, CA: SAGE Publications, Inc. Retrieved from http://sk.sagepub.com/reference/socialscience/n787.xml

Jones, D. R., Macias, C., Barreira, P. J., Fisher, W. H., Hargreaves, W. A., \& Harding, C. M. (2004). Prevalence, severity, and co-occurrence of chronic physical health problems of persons with serious mental illness. Psychiatric Services, 55(11), 1250-1257. doi:10.1176/appi.ps.55.11.1250

Khan, A., Faucett, J., Morrison, S., \& Brown, W. A. (2013). Comparative mortality risk in adult patients with schizophrenia, depression, bipolar disorder, anxiety disorders, and attention-deficit/hyperactivity disorder participating in psychopharmacology clinical trials. Journal of the American Medical Association - Psychiatry, 70(10), 1091-1099. doi:10.1001/jamapsychiatry.2013.149

Kilbourne, A. M., Biswas, K., Pirraglia, P. A., Sajatovic, M., Williford, W. O., \& Bauer, M. S. (2009). Is the collaborative chronic care model effective for patients with bipolar disorder and co-occurring conditions? Journal of Affective Disorders, 112(1-3), 256-261. doi:10.1016/j.jad.2008.04.010

Kilbourne, A. M., Lai, Z., Bowersox, N., Pirraglia, P., \& Bauer, M. S. (2011). Does colocated care improve access to cardiometabolic screening for patients with 
serious mental illness? General Hospital Psychiatry, 33, 634-636.

doi:10.1016/j.genhosppsych.2011.07.003

Kilbourne, A. M., Welsh, D., McCarthy, J. F., Post, E. P., \& Blow, F. C. (2008). Quality of care for cardiovascular disease-related conditions in patients with and without mental disorders. Journal of General Internal Medicine, 23(10), 1628-33. doi:0.1007/s11606-008-0720-z

Kraska, M. (2010). Quantitative research. In N. Salkind, Encyclopedia of research design. Thousand Oaks, CA: SAGE Publications, Inc. Retrieved from http://sk.sagepub.com/reference/researchdesign/n352.xml

Laerd Statistics. (2015a). Linear regression using SPSS Statistics [Statistical tutorials and software guides]. Retrieved from https://statistics.laerd.com/

Laerd Statistics. (2015b). Multiple regression using SPSS Statistics. [Statistical tutorials and software guides]. Retrieved from https://statistics.laerd.com/

Laerd Statistics. (2015c). Poisson regression using SPSS Statistics [Statistical tutorials and software guides]. Retrieved from https://statistics.laerd.com/

Lafeuille, M. H., Dean, J., Fastenau, J., Panish, J., Olsen, W., Duh, M. S., \& Lefebvre, P. (2014). Burden of schizophrenia on selected comorbidity costs. Expert Review of Pharmacoeconomics \& Outcomes Research, 14(2), 259-267. doi:10.1586/14737167.2014.894463

Laureate Education (Producer). (2009). Data cleaning and dealing with assumptions [Video file]. Baltimore, MD: Laureate Education, Inc. 
Laursen, T. M., Munk-Olsen, T., \& Gasse, C. (2011). Chronic somatic comorbidity and excess mortality due to natural causes in persons with schizophrenia or bipolar affective disorder. PLoS ONE, 6(9), e24597. doi:10.1371/journal.pone.0024597

Lichstein, J. C., Domino, M. E., Beadles, C. A., Ellis, A. R., Farley, J. F., Morrissey, J. P., ... Jackson, C. T. (2014). Use of medical homes by patients with comorbid physical and severe mental illness. Medical Care, 52 (3 Supp 2), S85-S91. doi: $10.1097 / \mathrm{mlr} .0000000000000025$

Li, Y., Glance, L. G., Cai, X., \& Mukamel, D. B. (2008). Mental illness and hospitalization for ambulatory care sensitive medical conditions. Medical Care, 46(12), 1249-1256. doi:10.1097/MLR.0b013e31817e188c

Malm, U. I., Ivarsson, B. Å. R., \& Allebeck, P. (2014). Durability of the efficacy of integrated care in schizophrenia: A five-year randomized controlled study. Psychiatric Services, 65(8), 1054-1057. doi:10.1176/appi.ps.201300164

Manderscheid, R., \& Kathol, R. (2014). Fostering sustainable, integrated medical and behavioral health services in medical settings. Annals of Internal Medicine, 160, 61-65. doi:10.7326/m13-1693

Mann, C. J. (2003). Observational research methods. Research design II: cohort, cross sectional, and case-control studies. Emergency Medicine Journal, 20(1), 54-60. doi:10.1136/emj.20.1.54

McCusker, J., Roberge, D., Lévesque, J. F., Ciampi, A., Vadeboncoeur, A., Larouche, D., \& Sanche, S. (2010). Emergency department visits and primary care among adults 
with chronic conditions. Medical Care, 48(11), 972-980.

doi:10.1097/MLR.0b013e3181eaf86d

McGinty, E. E., Baller, J., Azrin, S. T., Juliano-Bult, D., \& Daumit, G. L. (2016). Interventions to address medical conditions and health-risk behaviors among persons with serious mental illness: A comprehensive review. Schizophrenia Bulletin, 42(1), 96-124. doi:10.1093/schbul/sbv101

McGinty, E. E., \& Sridhara, S. (2014). Potentially preventable medical hospitalizations among Maryland residents with mental illness, 2005-2010. Psychiatric Services, 65(7), 951-953. doi:10.1176/appi.ps.201300323

McLellan, A. T., Starrels, J. L., Tai, B., Gordon, A. J., Brown, R., Ghitza, U., ... McNeeley, J. (2014). Can substance use disorders be managed using the chronic care model? Review and recommendations from a NIDA consensus group. Public Health Reviews, 35(2).

Mechanic, D. (2012). Seizing opportunities under the Affordable Care Act for transforming the mental and behavioral health system. Health Affairs, 31(2), 376382. doi:10.1377/hlthaff.2011.0623

Medici, C. R., Videbech, P., Gustafsson, L. N., \& Munk-Jørgensen, P. (2015). Mortality and secular trend in the incidence of bipolar disorder. Journal of Affective Disorders, 183, 39-44. doi:10.1016/j.jad.2015.04.032

Mental Health Cooperative. (2014). MHC at a glance. Retrieved from http://www.mhctn.org/about-mhc/mhc-glance 
Miller, C. J., Grogan-Kaylor, A., Perron, B. E., Kilbourne, A. M., Woltmann, E., \& Bauer, M. S. (2013). Collaborative chronic care models for mental health conditions: Cumulative meta-analysis and meta-regression to guide future research and implementation. Medical Care, 51(10), 922-930. doi:10.1097/MLR.0b013e3182a3e4c4

Minassian, A., Vilke, G. M., \& Wilson, M. P. (2013). Frequent emergency department visits are more prevalent in psychiatric, alcohol abuse, and dual diagnosis conditions than in chronic viral illnesses such as hepatitis and human immunodeficiency virus. Journal of Emergency Medicine, 45(4), 520-525. doi:10.1016/j.jemermed.2013.05.007

Mitchell, A. J., \& Lawrence, D. (2011). Revascularisation and mortality rates following acute coronary syndromes in people with severe mental illness: comparative meta-analysis. British Journal of Psychiatry, 198(6), 434-441. doi:10.1192/bjp.bp.109.076950

Mueser, K. T., Bartels, S. J., Santos, M., Pratt, S. I., \& Riera, E. G. (2012). Integrated illness management and recovery: A program for integrating physical and psychiatric illness self-management in older persons with severe mental illness. American Journal of Psychiatric Rehabilitation, 15(2), 131-156. doi: $10.1080 / 15487768.2012 .679558$

Nasrallah, H. A., \& Newcomer, J. W. (2004). Atypical antipsychotics and metabolic dysregulation: Evaluating the risk/benefit equation and improving the standard of 
care. Journal of Clinical Psychopharmacology, 24, S7-S14.

doi:10.1097/01.jcp.0000142282.62336.e9

Newcomer, J. W. (2007). Antipsychotic medications: Metabolic and cardiovascular risk. Journal of Clinical Psychiatry, 68(Suppl 4), 8-13.

Newhouse, J. P., Buntin, M. B., \& Chapman, J. D. (1997). Risk adjustment and Medicare: Taking a closer look. Health Affairs, 16(5), 26-43. doi:10.1377/hlthaff.16.5.26

Nicol, G. E., Campagna, E. J., Garfield, L. D., Newcomer, J. W., Parks, J. J., \& Morrato, E. H. (2015). The role of clinical setting and management approach in metabolic testing among Youths and adults treated with antipsychotics. Psychiatric Services, 67(1), 128-132. doi:10.1176/appi.ps.201400428

Norquist, G. S. (2014). Research in integrated care. In P. Summergrad \& R. G. Kathol (Eds.), Integrated care in psychiatry: Redefining the role of mental health professionals in the medical setting. New York: Springer.

Nussbaum, E. M., Elsadat, S., \& Khago, A. H. (2008). Chapter 21. Best practices in analyzing count data: Poisson regression. In J. Osborne, Best practices in quantitative methods (pp. 306-323). Thousand Oaks, CA: SAGE Publications, Inc. Retrieved from http://jwosborne.com/bp_ch21.html

Olfson, M., Gerhard, T., Huang, C., Crystal, S., \& Stroup, T. S. (2015). Premature mortality among adults with schizophrenia in the United States. JAMA Psychiatry, 72(12), 1172-1181. doi:10.1001/jamapsychiatry.2015.1737 
Oster, A., \& Bindman, A. B. (2003). Emergency department visits for ambulatory care sensitive conditions: Insights into preventable hospitalizations. Medical Care, 41(2), 198-207. doi:10.1097/01.mlr.0000045021.70297.9f

Perkins, D. (2015). Integrating mental and physical health care: Lessons from the Second World Congress on Integrated Care in Sydney. International Journal of Integrated Care, 15(1). doi:10.5334/ijic.2011

Perlin, J. B., Kolodner, R. M., \& Roswell, R. H. (2004). The Veterans Health Administration: Quality, value, accountability, and information as transforming strategies for patient-centered care. American Journal of Managed Care, $10(11 \mathrm{Pt}$ 2), 828-836. doi:10.12927/hcpap..17381

Pincus, H. A., Jun, M., Franx, G., van der Feltz-Cornelis, C., Ito, H., \& Mossialos, E. (2015). How can we link general medical and behavioral health care? International models for practice and policy. Psychiatric Services, 66(8), 775777. doi:10.1176/appi.ps.201400461

Pirraglia, P. A., Kilbourne, A. M., Lai, Z., Friedmann, P. D., \& O’Toole, T. P. (2011). Colocated general medical care and preventable hospital admissions for veterans with serious mental illness. Psychiatric Services, 62(5), 554-557. doi:10.1176/appi.ps.62.5.554

Pirraglia, P. A., Rowland, E., Wu, W.-C., Friedmann, P. D., O’Toole, T. P., Cohen, L. B., \& Taviera, T. H. (2012). Benefits of a primary care clinic collocated and integrated in a mental health setting for veterans with serious mental illness. Preventing Chronic Disease, 9(E51). doi.10.5888/pcd9.110113 
Pomerantz, A. S., Shiner, B., Watts, B. V., Detzer, M. J., Kutter, C., Street, B., \& Scott, D. (2010). The White River model of colocated collaborative care: A platform for mental and behavioral health care in the medical home. Families, Systems \& Health: The Journal of Collaborative Family Healthcare, 28(2), 114-129. doi:10.1037/a0020261

Powell, B. J., McMillen, J. C., Proctor, E. K., Carpenter, C. R., Griffey, R. T., Bunger, A. C., ... York, J. L. (2012). A compilation of strategies for implementing clinical innovations in health and mental health. Medical Care Research and Review, 69(2), 123-157. doi:10.1177/1077558711430690

Quan, H., Sundararajan, V., Halfon, P., Fong, A., Burnand, B., Luthi, J.-C., ... Ghali, W. A. (2005). Coding algorithms for defining comorbidities in ICD-9-CM and ICD10 administrative data. Medical Care, 43(11), 1130-1139. doi:10.1097/01.mlr.0000182534.19832.83

Randall, I., Mohr, D. C., \& Maynard, C. (2014). VHA patient-centered medical home associated with lower rate of hospitalizations and specialty care among veterans with posttraumatic stress disorder. Journal for Healthcare Quality. doi:10.1111/jhq.12092.

Regier, D. A., Narrow, W. E., Rae, D. S., Manderscheid, R. W., Locke, B. Z., \& Goodwin, F. K. (1993). The de facto mental and addictive disorders service system. Epidemiologic Catchment Area prospective 1-year prevalence rates of disorders and services. Archives of General Psychiatry, 50(2), 85-94. doi:10.1001/archpsyc.1993.01820140007001 
Reiss-Brennan, B. (2014). Mental health integration normalizing team care. Journal of Primary Care \& Community Health, 5(1), 55-60. doi: $10.1177 / 2150131913508983$

Rush, B. (2014). Evaluating the complex: Alternative models and measures for evaluating collaboration among substance use services with mental health, primary care and other services and sectors. Nordic Studies on Alcohol and Drugs, 31, 27-44. doi:10.2478/nsad-2014-0003

Substance Abuse and Mental Health Services Administration. (2013). Results from the 2012 National Survey on Drug Use and Health: Mental health findings (NSDUH Series H-47 No. SMA 13-4805). Rockville, MD: Substance Abuse and Mental Health Services Administration.

Substance Abuse and Mental Health Services Administration. (2014, December 19). Primary and behavioral health care integration. Retrieved July 30, 2015, from http://www.samhsa.gov/grants/grant-announcements/sm-15-005.

Salsberry, P. J., Chipps, E., \& Kennedy, C. (2005). Use of general medical services among Medicaid patients with severe and persistent mental illness. Psychiatric Services, 56, 458-462. doi:10.1176/appi.ps.56.4.458

Schaps, M., \& Post, S. (2015). Best practices in behavioral-physical health integration. Illinois: Health and Medicine Policy Working Group. Retrieved from hmprg.org Scott, D., Platania-Phung, C., \& Happell, B. (2012). Quality of care for cardiovascular disease and diabetes amongst individuals with serious mental illness and those 
using antipsychotic medications. Journal for Healthcare Quality, 34(5), 15-21. doi:10.1111/j.1945-1474.2011.00155.x

Shanmugam, R. (2007). Poisson distribution. In N. Salkind, Encyclopedia of measurement and statistics, Vol 3, (pp. 773-776). Thousand Oaks, CA: SAGE Publications, Inc. doi:10.4135/9781412952644.n350

Society of Thorasic Surgeons. (2015). What is risk adjustment? Retrieved October 12, 2015, from http://www.sts.org/patient-information/what-risk-adjustment Solberg, L. I., Crain, A. L., Jaeckels, N., Ohnsorg, K. A., Margolis, K. L., Beck, A., ... Van de Ven, A. H. (2013). The DIAMOND initiative: Implementing collaborative care for depression in 75 primary care clinics. Implementation Science, 8, 135. doi:10.1186/1748-5908-8-135

Tang, N., Stein, J., Hsia, R. Y., Maselli, J. H., \& Gonzales, R. (2010). Trends and characteristics of US emergency department visits, 1997-2007. Journal of the American Medical Association, 304(6), 664-670. doi:10.1001/jama.2010.1112

Thiejke, S., Vannoy, S., \& Unutzer, J. (2007). Integrating mental health and primary care. Primary Care Clinical Office Practice, 34, 571-592. doi:10.1016/j.pop.2007.05.007

Thornicroft, G. (2011). Physical health disparities and mental illness: the scandal of premature mortality. British Journal of Psychiatry, 199, 441-442. doi:10.1192/bjp.bp.111.092718

Thota, A. B., Sipe, T. A., Byard, G. J., Zometa, C. S., Hahn, R. A., McKnight-Eily, L. R., ... Williams, S. P. (2012). Collaborative care to improve the management of 
depressive disorders: A community guide systematic review and meta-analysis. American Journal of Preventive Medicine, 42(5), 525-538.

doi:10.1016/j.amepre.2012.01.019

Treatment Advocacy Center. (2015). No room at the inn. Retrieved December 26, 2015, from http://tacreports.org/bed-study

Trochim, W. M. K. (2006). Sampling. In W. M. K. Trochim: Research methods knowledge base. Retrieved from http://www.socialresearchmethods.net/kb/sampling.php

Tsasis, P., Evans, J. M., \& Owen, S. (2012). Reframing the challenges to integrated care: A complex-adaptive systems perspective. International Journal of Integrated Care, 12. doi:10.5334/ijic. 843

Tsasis, P., Evans, J. M., Rush, L., \& Diamond, J. (2013). Learning to learn: Towards a relational and transformational model of learning for improved integrated care delivery. Administrative Sciences, 3(2), 9-31. doi:10.3390/admsci3020009

United States Department of Health and Human Services. (n.d.-a). Health information privacy. Retrieved February 6, 2015, from http://www.hhs.gov/ocr/privacy/

United States Department of Health and Human Services. (n.d.-b). What are federally qualified health centers (FQHCs)? Retrieved September 27, 2015, from http://www.hrsa.gov/healthit/toolbox/RuralHealthITtoolbox/Introduction/qualifie d.html 
Veterans Health Administration. (2015a). About VA Mental Health - Mental Health

[General Information]. Retrieved February 17, 2016, from

http://www.mentalhealth.va.gov/VAMentalHealthGroup.asp

Veterans Health Administration. (2015b). Veterans Health Administration [Homepage].

Retrieved February 17, 2016, from http://www.va.gov/health/

Vijayaraghavan, M., Messer, K., Xu, Z., Sarkin, A., \& Gilmer, T. P. (2015). Psychiatric readmissions in a community-based sample of patients with mental disorders. Psychiatric Services, 66(5), 551-554. doi:10.1176/appi.ps.201400092

Wagner, E. H., Austin, B. T., \& Von Korff, M. (1996). Organizing care for patients with chronic illness. Milbank Quarterly, 74(4), 511-544. doi:10.2307/3350391

Wagner, E. H., Glasgow, R. E., Davis, C., Bonomi, A. E., Provost, L., McCulloch, D., ... Sixta, C. (2001). Quality improvement in chronic illness care: A collaborative approach. Joint Commission Journal on Quality and Patient Safety, 27(2), 63-80. doi:10.1016/s1070-3241(01)27007-2

Walden University. (2014). Understanding power and effect size: A practical overview. Laureate Education, Inc. Retrieved from www.waldeu.edu

Walker, E. R., McGee, R. E., \& Druss, B. G. (2015). Mortality in mental disorders and global disease burden implications: A systematic review and meta-analysis. JAMA Psychiatry, 72(4), 334-341. doi:10.1001/jamapsychiatry.2014.2502

Woltmann, E., Grogan-Kaylor, A., Perron, B., Georges, H., Kilbourne, A. M., \& Bauer, M. S. (2012). Comparative effectiveness of collaborative chronic care models for mental health conditions across primary, specialty, and behavioral health care 
settings: Systematic review and meta-analysis. American Journal of Psychiatry, 169(8), 790-804. doi:10.1176/appi.ajp.2012.11111616

Wright, B., Potter, A. J., \& Trivedi, A. (2015). Federally qualified health center use among dual eligibles: Rates of hospitalizations and emergency department visits. Health Affairs, 34(7), 1147-1155. doi:10.1377/hlthaff.2014.0823

Yang, C. C., Chen, P. C., Hsu, C. W., Chang, S. L., \& Lee, C. C. (2015). Validity of the age-adjusted Charlson Comorbidity Index on clinical outcomes for patients with nasopharyngeal cancer post radiation treatment: A 5-year nationwide cohort study. PLoS ONE, 10(1). doi:10.1371/journal.pone.0117323

Yilmaz, K. (2013). Comparison of quantitative and qualitative research traditions: Epistemological, theoretical, and methodological differences. European Journal of Education, 48(2), 311-325. doi:10.1111/ejed.12014

Zeller, S., Calma, N., \& Stone, A. (2014). Effects of a dedicated regional psychiatric emergency service on boarding of psychiatric patients in area emergency departments. Western Journal of Emergency Medicine, 15(1), 1-6. doi:10.5811/westjem.2013.6.17848 
Appendix A: Business Associate Agreement (Redacted)

\section{Health information privacy addendum}

This Addendum is made as of May 18, 2015, by and between [REDACTED] ("Covered Entity") and HEIDI WATERS (“Business Associate").

\section{RECITALS:}

A. Business Associate and Covered Entity have entered into an agreement or agreements pursuant to which Business Associate provides certain services to Covered Entity. Such agreement or agreements are collectively referred to herein as the "Agreement."

B. To enable Business Associate to carry out its obligations under the Agreement, Business Associate may, on the terms set forth herein, create, receive, maintain, or transmit from or on behalf of Covered Entity, Individually Identifiable Health Information, as such term is defined in 45 C.F.R. $\S 160.103$.

C. Pursuant to 45 C.F.R. Part C (the "Security Rule") and 45 C.F.R. Part E (the "Privacy Rule") Covered Entity is required to enter into a contract with Business Associate to ensure that Business Associate appropriately safeguards such information.

D. Covered Entity and Business Associate desire to make this Addendum to the Agreement in order to enable Covered Entity to satisfy its obligations under the Security Rule and the Privacy Rule.

NOW, THEREFORE, for and in consideration of the mutual promises herein contained and other good and valuable consideration, the receipt and sufficiency of which are hereby acknowledged, the parties hereto agree as follows: 


\section{Definitions.}

"Individual" shall have the same meaning as the term "individual" in 45 C.F.R. $§ 160.103$ and shall include a person who qualifies as a personal representative in accordance with 45 C.F.R. $\S 164.502(\mathrm{~g})$.

"Protected Health Information" shall have the same meaning as the term "protected health information" in 45 C.F.R. $\S 160.103$, limited to the information created, received, maintained, or transmitted by Business Associate from or on behalf of Covered Entity. “Representatives" means Business Associate's directors, officers, employees, subcontractors and agents.

Terms used in this Addendum and not otherwise defined herein shall have the same meaning of those terms as they are used in the Security Rule, the Privacy Rule, and the Breach Notification Rule (as defined herein).

\section{Obligations and Activities of Business Associate}

Confidentiality. Business Associate agrees to not use or disclose, and to prevent its Representatives from using or disclosing, Protected Health Information other than as permitted or required by this Addendum or as Required By Law.

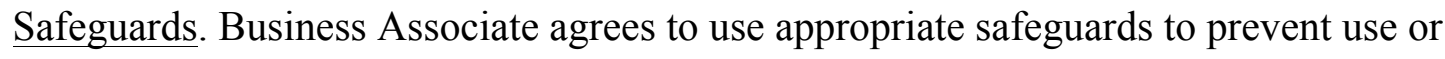
disclosure of the Protected Health Information other than as provided for by this Addendum.

Mitigation. Business Associate agrees to mitigate, to the extent practicable, any harmful effect that is known to Business Associate of a use or disclosure of Protected Health 
Information by Business Associate or its Representatives in violation of the requirements of this Addendum.

Reporting. Business Associate agrees to promptly report to Covered Entity's Privacy Officer any use or disclosure of the Protected Health Information not provided for by this Addendum or by an agreement required by Section 0 of this Addendum of which it becomes aware, including any breach of Unsecured Protected Health Information as required by 45 C.F.R. $\S 164.410$.

Agents and Subcontractors. Business Associate agrees to ensure that any agent, including without limitation a subcontractor, that creates, receives, maintains, or transmits Protected Health Information on behalf of Business Associate agrees to the same restrictions and conditions that apply through this Addendum to Business Associate with respect to such information, pursuant to and in accordance with a written contract, as required by 45 C.F.R. $\S 502(e)(2)$.

Access and Amendment. Business Associate agrees to provide access, at the request of Covered Entity, and in the time and manner reasonably designated by Covered Entity, to Protected Health Information in a Designated Record Set, to Covered Entity in order to meet the requirements under 45 C.F.R. $\S 164.524$. Business Associate agrees to make any amendment(s) to Protected Health Information in a Designated Record Set that Covered Entity directs or agrees to pursuant to 45 C.F.R. $\S 164.526$ at the request of Covered Entity and in the time and manner reasonably designated by Covered Entity. Books and Records. Business Associate agrees to make its internal practices, books, and records, including policies and procedures and Protected Health Information, relating to 
the use and disclosure of Protected Health Information available to Covered Entity, or to the Secretary, in a time and manner reasonably requested by Covered Entity or designated by the Secretary, for purposes of the Secretary determining Covered Entity's compliance with the Privacy Rule. If the Secretary requests such access, Business Associate shall promptly notify Covered Entity’s Privacy Officer and provide the Privacy Officer with a copy of such request. Business Associate shall consult and cooperate with Covered Entity concerning the proper response to such request and shall provide Covered Entity with a copy of each book, document and record made available to the Secretary or shall identify each such book, document, and record and grant Covered Entity access thereto for review and copying. Notwithstanding the foregoing, nothing in this section shall be deemed to require Business Associate to waive the attorney-client, accountantclient, or other legal privilege, and nothing in this section shall impose upon Covered Entity any obligation to review Business Associate's practices, books or records. Accounting. Business Associate agrees to document such disclosures of Protected Health Information and information related to such disclosures as would be required for Covered Entity to respond to a request by an Individual for an accounting of disclosures of Protected Health Information in accordance with 45 C.F.R. $\S 164.528$. Business Associate agrees to provide to Covered Entity, in a time and manner reasonably designated by Covered Entity, information collected in accordance with this section to permit Covered Entity to respond to a request by an Individual for an accounting of disclosures of Protected Health Information in accordance with 45 C.F.R. $\S 164.528$. 
Uses and Disclosures Required By Law. Except to the extent prohibited by law, Business Associate shall immediately notify Covered Entity’s Privacy Officer if it receives a request for disclosure of Protected Health Information with which Business Associate believes it is Required By Law to comply and disclosure pursuant to which would not otherwise be permitted by this Addendum. Business Associate shall provide Covered Entity's Privacy Officer with a copy of such request, shall consult and cooperate with Covered Entity concerning the proper response to such request, and shall provide Covered Entity with a copy of any information disclosed pursuant to such request. Electronic Protected Health Information. With regard to Electronic Protected Health Information, Business Associate shall: (i) comply with the applicable requirements of the Security Rule and implement administrative, physical, and technical safeguards that reasonably and appropriately protect the confidentiality, integrity and availability of such information that Business Associate creates, receives, maintains or transmits on behalf of Covered Entity; (ii) ensure that any agent, including a subcontractor, that creates, receives, maintains, or transmits Electronic Protected Health Information on behalf of Business Associate agrees to comply with the applicable requirements of the Security Rule by entering into a contract or other arrangement that complies with 45 C.F.R. $\S$ 164.314; and (iii) report to Covered Entity any Security Incident of which Business Associate becomes aware. Business Associate's obligations under this section are in addition to its obligations under Section 0 of this Addendum.

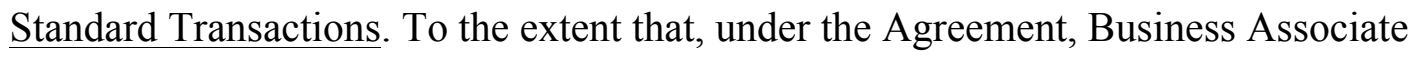
conducts on behalf of Covered Entity all or part of a Transaction (as defined in 45 C.F.R. 
Parts 160 and 162 (the "Electronic Transactions Rule")), Business Associate shall comply with, and shall cause any of its agents or subcontractors to comply with, the Electronic Transactions Rule.

\section{Permitted Uses and Disclosures of Protected Health Information by Business}

\section{Associate}

Use or Disclosure to Provide Services Under the Agreement. Except as otherwise limited in this Addendum, Business Associate may use or disclose Protected Health Information to perform functions, activities, or services for, or on behalf of, Covered Entity as specified in the applicable Agreement, provided that such use or disclosure would not violate the Privacy Rule if done by Covered Entity or the minimum necessary policies and procedures of Covered Entity. To the extent Business Associate is to carry out any of Covered Entity's obligations under the Privacy Rule pursuant to the terms of this Addendum, Business Associate shall comply with the requirements of the Privacy Rule that apply to Covered Entity in the performance of such obligation.

Use or Disclosure for Business Associate's Management and Administration. Except as otherwise limited in this Addendum, Business Associate may use Protected Health Information for the proper management and administration of Business Associate or to carry out the legal responsibilities of Business Associate. Except as otherwise limited in this Addendum, Business Associate may disclose Protected Health Information for the proper management and administration of Business Associate, provided that such disclosures are Required By Law, or Business Associate obtains reasonable assurances from the person to whom the information is disclosed that it will remain confidential and 
used or further disclosed only as Required By Law or for the purpose for which it was disclosed to the person, and the person notifies the Business Associate of any instances of which it is aware in which the confidentiality of the information has been breached. Use or Disclosure to Provide Data Aggregation Services. Except as otherwise limited in this Addendum and if authorized by the Agreement or with the written permission of Covered Entity, Business Associate may de-identify Protected Health Information or use Protected Health Information to provide Data Aggregation services to Covered Entity as permitted by 42 C.F.R. $\S 164.504(\mathrm{e})(2)(\mathrm{i})(\mathrm{B})$.

\section{Responsibilities of covered entity}

Notice of Privacy Practices. Covered Entity shall notify Business Associate of any limitation(s) in the notice of privacy practices of Covered Entity in accordance with 45 C.F.R. $\S 164.520$, to the extent that such limitation may affect Business Associate's use or disclosure of Protected Health Information.

Change or Revocation of Permission. Covered Entity shall notify Business Associate of any changes in, or revocation of, permission by an Individual to use or disclose Protected Health Information, to the extent that such changes may affect Business Associate's use or disclosure of Protected Health Information.

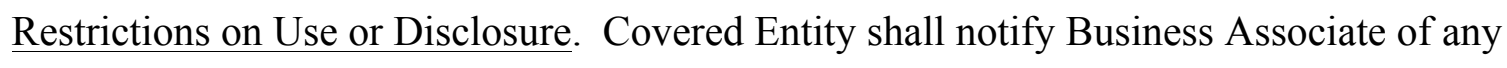
restriction to the use or disclosure of Protected Health Information that Covered Entity has agreed to in accordance with 45 C.F.R. $\S 164.522$, to the extent that such restriction may affect Business Associate's use or disclosure of Protected Health Information. 
Permissible Requests. Covered Entity shall not request Business Associate to use or disclose Protected Health Information in any manner that would not be permissible under this Addendum.

\section{Term and Termination}

Term. The Term of this Addendum shall be effective as of the date on which the parties first entered into an Agreement, and shall expire when all of the Protected Health Information provided by Covered Entity to Business Associate, or created or received by Business Associate on behalf of Covered Entity, is destroyed or returned to Covered Entity, or, if it is infeasible to return or destroy Protected Health Information, protections are extended to such information, in accordance with the termination provisions in Section 0 of this Addendum.

Termination. If Covered Entity determines that Business Associate or any Representative of Business Associate has violated a material term of this Addendum, Covered Entity may either: (i) Provide an opportunity for Business Associate to cure the violation and terminate, without penalty, this Addendum and any Agreement if Business Associate does not cure the violation within the time specified by Covered Entity; (ii) Immediately terminate, without penalty, this Addendum and any Agreement if Business Associate has violated a material term of this Addendum; or (iii) If neither termination nor cure are feasible, Covered Entity may report the violation to the Secretary.

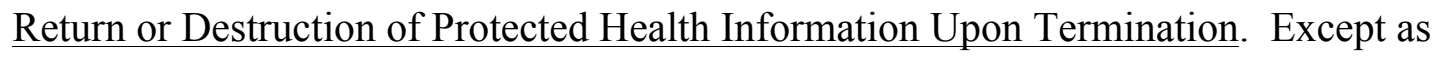
provided below, upon termination for any reason of this Addendum, Business Associate shall return or destroy all Protected Health Information, including any Electronic 
Protected Health Information. This provision shall apply to Protected Health Information that is in the possession of subcontractors or agents of Business Associate. Business Associate shall retain no copies of the Protected Health Information. In the event that Business Associate determines that returning or destroying the Protected Health Information is infeasible, Business Associate shall provide to Covered Entity notification of the conditions that make return or destruction infeasible. Business Associate shall extend the protections of this Addendum to such Protected Health Information and limit further uses and disclosures of such Protected Health Information to those purposes that make the return or destruction infeasible, for so long as Business Associate maintains such Protected Health Information.

\section{Notification of breach of Unsecured Protected Health Information}

Definitions. Capitalized terms used in this Article VII and not otherwise defined herein have the meaning given to them in 45 C.F.R. Part 164, Subpart D (the "Breach Notification Rule").

Notification Following the Discovery of a Breach. Business Associate shall notify Covered Entity without unreasonable delay in the event of a Breach or suspected Breach of Unsecured Protected Health Information created, received, maintained or transmitted by Business Associate or its agents or subcontractors. In no event shall Business Associate notify Covered Entity later than ten (10) calendar days after its discovery of such a Breach or suspected Breach. For the purpose of this Article VI, a Breach or suspected Breach is deemed to be discovered by Business Associate as provided in 45 C.F.R. $\S 164.410$. 
Cooperation in Investigation. Covered Entity and Business Associate shall cooperate reasonably with each other and with their respective employees, agents, consultants, advisors, legal counsel or other representatives in connection with any investigation by either of them of a Breach or suspected Breach of Unsecured Protected Health Information created, received, maintained or transmitted by Business Associate or its agents or subcontractors and shall furnish upon request to each other such further information as the other party may reasonably request to carry out such investigation. Assistance in Proceedings. Business Associate will cooperate with Covered Entity and its counsel in the contest or defense of, and make available its personnel and provide any testimony and access to its books and records in connection with, any Proceeding involving or relating to a Breach or alleged Breach of Unsecured Protected Health Information created, received, maintained or transmitted by Business Associate or its agents or subcontractors. For the purposes of this Section, a "Proceeding" means any action, arbitration, audit, hearing, investigation, litigation or suit (whether civil, criminal, administrative, judicial or investigative, whether formal or informal, whether public or private) commenced, brought, conducted or heard by or before, or otherwise involving, any governmental body or arbitrator.

$\underline{\text { Reimbursement of Expenses. Business Associate will reimburse Covered Entity for any }}$ expense reasonably incurred by Covered Entity in connection with the provision of notice, as required by 45 C.F.R. $\S \S 164.404,164.406$ or 164.408 , of a Breach of Unsecured Protected Health Information created, received, maintained or transmitted by Business Associate or its agents or subcontractors. 


\section{Modifications to Comply with Standards}

In the event that additional standards are promulgated under the Health Insurance Portability and Accountability Act of 1996 or the Health Information Technology for Economic and Clinical Health Act, or any existing standards are amended, including without limitation the Privacy Rule, the Security Rule or the Breach Notification Rule, the parties agree to enter into a mutually acceptable amendment to this Addendum to enable Covered Entity to satisfy its obligations under such additional or amended standard(s).

\section{Miscellaneous}

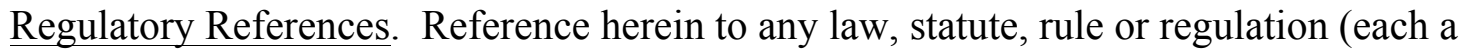
"Legal Requirement") means such Legal Requirement as amended, modified, codified, replaced or reenacted, in whole or in part, and in effect from time to time, including rules and regulations promulgated thereunder, and reference herein to any section or other provision of any Legal Requirement means that provision of such Legal Requirement from time to time in effect and constituting the substantive amendment, modification, replacement or reenactment of such section or other provision.

Survival. The respective rights and obligations of the parties under Section 0, Article VI and Section 0 of this Addendum shall survive the termination of this Addendum. The respective rights and obligations of Business Associate under Section 0 of this Addendum shall survive the termination or expiration of this Addendum for six (6) years from the date of the last disclosure of Protected Health Information by Business Associate for which Covered Entity is required to account under 45 C.F.R. $\S 164.528$. 
Ownership of Protected Health Information. The parties agree and acknowledge that, as between Covered Entity and Business Associate, Covered Entity is the owner of the Protected Health Information.

Injunctive Relief. Business Associate understands and acknowledges that any use or disclosure of Protected Health Information in violation of this Addendum will cause Covered Entity irreparable harm, the amount of which may be difficult to ascertain, and therefore agrees that Covered Entity shall have the right to apply to a court of competent jurisdiction for specific performance and/or an order restraining and enjoining any such further use, disclosure or breach and for such other relief as Covered Entity shall deem appropriate. Such right of Covered Entity is to be in addition to the remedies otherwise available to Covered Entity at law or in equity. Business Associate expressly waives the defense that a remedy in damages will be adequate and further waives any requirement in an action for specific performance or injunction for the posting of a bond by Covered Entity.

Limitation of Liability. Business Associate agrees that no provision of the Agreement purporting to limit Covered Entity's remedies under any legal or equitable theory shall limit Covered Entity's remedies with respect to a breach of this Addendum by Business Associate or its Representatives.

Amendment. This Addendum may be amended only by written agreement between the parties.

Interpretation. The headings of sections in this Addendum are for reference only and shall not affect the meaning of this Addendum. Any ambiguity in this Addendum shall 
be resolved to permit Covered Entity to comply with the Privacy Rule. In the event that a provision of this Addendum conflicts with a provision of the Agreement, the provision of this Addendum shall control, except to the extent that the Agreement places additional restrictions on Business Associate's use and disclosure of Protected Health Information. Otherwise, this Addendum shall be construed under, and in accordance with, the terms of the Agreement. This Addendum shall be interpreted by and construed in accordance with the laws of the State of [REDACTED].

No Third Party Beneficiaries. Nothing express or implied in this Addendum is intended to confer, nor shall anything herein confer, upon any person other than the parties and the respective successors and assigns of the parties any rights, remedies, obligations, or liabilities whatsoever.

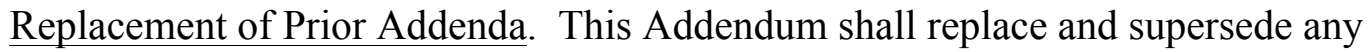
previously entered into Business Associate Agreement between the parties addressing the same subject matter.

IN WITNESS WHEREOF, the parties hereto have executed this Addendum as of the day and year first written above.

\section{[REDACTED]}

By:

Title:

\section{HEIDI WATERS}

By:

Title: 


\section{Appendix B: Letter of Cooperation}

\section{Letter of Cooperation}

February 10, 2016

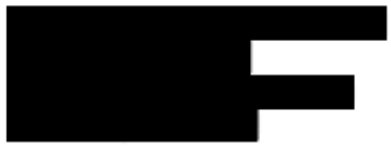

Dear Heidi Waters,

Based on my review of your research proposal, I give permission for you to conduct the study entitled: Evaluating the Impact of Integrated Healthcare on Service Utilization in Serious Mental Illness within the . As part of this study, I authorize you to analyze administrative data (e.g. consumer contact data, authorization data and/or electronic medical record data) for members of $\square$ that will be provided to you by $\square$. I also authorize you to use the results for your dissertation and publish the results in peer-reviewed journals.

You and have already signed a Business Associate Agreement, in compliance with the Health Insurance Portability and Accountability Act (HIPAA) of 1996. We understand that our organization's responsibilities include: providing administrative data (e.g. consumer contact data, authorization data and/or electronic medical record data) and information about the Integrated Health Clinic within $\square$. We reserve the right to withdraw from the study at any time if our circumstances change.

I confirm that I am authorized to approve research in this setting and that this plan complies with the organization's policies.

I understand that the data collected will remain entirely confidential and may not be provided to anyone outside of the student's supervising faculty/staff without permission from the Walden University IRB.

Sincerely,

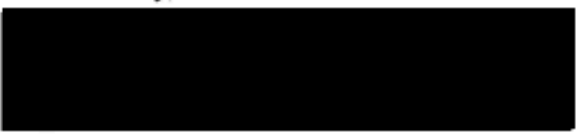


Appendix C: Confidentiality Agreement

CONFIDENTIALITY AGREEMENT

\section{Name of Signer:}

Heidi C. Waters

During the course of my activity in collecting data for this research: Evaluating the Impact of Integrated Care on Service Utilization in Serious Mental Illness, I will have access to information that is confidential and should not be disclosed. I acknowledge that the information must remain confidential, and that improper disclosure of confidential information can be damaging to the participant.

By signing this Confidentiality Agreement I acknowledge and agree that:

1. I will not disclose or discuss any confidential information with others, including friends or family.

2. I will not in any way divulge, copy, release, sell, loan, alter or destroy any confidential information except as properly authorized.

3. I will not discuss confidential information where others can overhear the conversation. I understand that it is not acceptable to discuss confidential information even if the participant's name is not used.

4. I will not make any unauthorized transmissions, inquiries, modification or purging of confidential information.

5. I agree that my obligations under this agreement will continue after termination of the job that I will perform.

6. I understand that violation of this agreement will have legal implications. 
7. I will only access or use systems or devices I'm officially authorized to access and I will not demonstrate the operation or function of systems or devices to unauthorized individuals.

Signing this document, I acknowledge that I have read the agreement and I agree to comply with all the terms and conditions stated above.

Signature

Date 
Appendix D: Data Use Agreement

\section{DATA USE AGREEMENT}

This Data Use Agreement (“Agreement”), effective as of Enter Date (“Effective Date"), is entered into by and between Heidi C. Waters ("Data Recipient") and [REDACTED] (“Data Provider"). The purpose of this Agreement is to provide Data Recipient with access to a Limited Data Set ("LDS") for use in research in accord with laws and regulations of the governing bodies associated with the Data Provider, Data Recipient, and Data Recipient's educational program. In the case of a discrepancy among laws, the agreement shall follow whichever law is more strict. Definitions. Due to the study's affiliation with Laureate, a USA-based company, unless otherwise specified in this Agreement, all capitalized terms used in this Agreement not otherwise defined have the meaning established for purposes of the USA "HIPAA Regulations" and/or "FERPA Regulations" codified in the United States Code of Federal Regulations, as amended from time to time.

Preparation of the LDS. Data Provider shall prepare and furnish to Data Recipient a LDS in accord with any applicable laws and regulations of the governing bodies associated with the Data Provider, Data Recipient, and Data Recipient's educational program.

Data Fields in the LDS. No direct identifiers such as names may be included in the Limited Data Set (LDS). In preparing the LDS, Data Provider shall include the data fields specified as follows, which are the minimum necessary to accomplish 
the research: list of clients served by the Data Provider including demographic information (date of birth, gender, primary diagnosis), inpatient authorization data (including authorization number, date of admission, date of discharge, facility, primary diagnosis upon admission), emergency department utilization files (including authorization number, date, facility, primary diagnosis for visit), list of clients enrolled in the Integrated Health Clinic (IHC).

Responsibilities of Data Recipient. Data Recipient agrees to:

Use or disclose the LDS only as permitted by this Agreement or as required by law;

Use appropriate safeguards to prevent use or disclosure of the LDS other than as permitted by this Agreement or required by law;

Report to Data Provider any use or disclosure of the LDS of which it becomes aware that is not permitted by this Agreement or required by law;

Require any of its subcontractors or agents that receive or have access to the LDS to agree to the same restrictions and conditions on the use and/or disclosure of the LDS that apply to Data Recipient under this Agreement; and

Not use the information in the LDS to identify or contact the individuals who are data subjects.

Permitted Uses and Disclosures of the LDS. Data Recipient may use and/or disclose the LDS for its Research activities only. 
Term and Termination.

Term. The term of this Agreement shall commence as of the Effective Date and shall continue for so long as Data Recipient retains the LDS, unless sooner terminated as set forth in this Agreement.

Termination by Data Recipient. Data Recipient may terminate this agreement at any time by notifying the Data Provider and returning or destroying the LDS.

Termination by Data Provider. Data Provider may terminate this agreement at any time by providing thirty (30) days prior written notice to Data Recipient.

For Breach. Data Provider shall provide written notice to Data Recipient within ten (10) days of any determination that Data Recipient has breached a material term of this Agreement. Data Provider shall afford Data Recipient an opportunity to cure said alleged material breach upon mutually agreeable terms. Failure to agree on mutually agreeable terms for cure within thirty (30) days shall be grounds for the immediate termination of this Agreement by Data Provider.

Effect of Termination. Sections 1, 4, 5, 6(e) and 7 of this Agreement shall survive any termination of this Agreement under subsections $\mathrm{c}$ or $\mathrm{d}$.

Miscellaneous.

Change in Law. The parties agree to negotiate in good faith to amend this Agreement to comport with changes in federal law that materially alter either or both parties' 
obligations under this Agreement. Provided however, that if the parties are unable to agree to mutually acceptable amendment(s) by the compliance date of the change in applicable law or regulations, either Party may terminate this Agreement as provided in section 6.

Construction of Terms. The terms of this Agreement shall be construed to give effect to applicable federal interpretative guidance regarding the HIPAA Regulations.

No Third Party Beneficiaries. Nothing in this Agreement shall confer upon any person other than the parties and their respective successors or assigns, any rights, remedies, obligations, or liabilities whatsoever.

Counterparts. This Agreement may be executed in one or more counterparts, each of which shall be deemed an original, but all of which together shall constitute one and the same instrument.

Headings. The headings and other captions in this Agreement are for convenience and reference only and shall not be used in interpreting, construing or enforcing any of the provisions of this Agreement.

IN WITNESS WHEREOF, each of the undersigned has caused this Agreement to be duly executed in its name and on its behalf.

DATA PROVIDER

Signed:
DATA RECIPIENT

Signed: 
Print Name:

Print Title:
Print Name:

Print Title: 